\title{
User's Guide for TOUGH2-MP - \\ A Massively Parallel Version of the TOUGH2 Code
}

Keni Zhang, Yu-Shu Wu, and Karsten Pruess

Earth Sciences Division

Lawrence Berkeley National Laboratory

May 2008 


\begin{abstract}
TOUGH2-MP is a massively parallel (MP) version of the TOUGH2 code, designed for computationally efficient parallel simulation of isothermal and nonisothermal flows of multicomponent, multiphase fluids in one, two, and three-dimensional porous and fractured media. In recent years, computational requirements have become increasingly intensive in large or highly nonlinear problems for applications in areas such as radioactive waste disposal, $\mathrm{CO}_{2}$ geological sequestration, environmental assessment and remediation, reservoir engineering, and groundwater hydrology. The primary objective of developing the parallel-simulation capability is to significantly improve the computational performance of the TOUGH2 family of codes. The particular goal for the parallel simulator is to achieve orders-of-magnitude improvement in computational time for models with ever-increasing complexity.
\end{abstract}

TOUGH2-MP is designed to perform parallel simulation on multi-CPU computational platforms. An earlier version of TOUGH2-MP (V1.0) was based on the TOUGH2 Version 1.4 with EOS3, EOS9, and T2R3D modules, a software previously qualified for applications in the Yucca Mountain project, and was designed for execution on CRAY T3E and IBM SP supercomputers. The current version of TOUGH2-MP (V2.0) includes all fluid property modules of the standard version TOUGH2 V2.0. It provides computationally efficient capabilities using supercomputers, Linux clusters, or multi-core PCs, and also offers many user-friendly features. The parallel simulator inherits all process capabilities from V2.0 together with additional capabilities for handling fractured media from V1.4.

This report provides a quick starting guide on how to set up and run the TOUGH2-MP program for users with a basic knowledge of running the (standard) version TOUGH2 code, The report also gives a brief technical description of the code, including a discussion of parallel methodology, code structure, as well as mathematical and numerical methods used. To familiarize users with the parallel code, illustrative sample problems are presented. 


\section{TABLE OF CONTENTS}

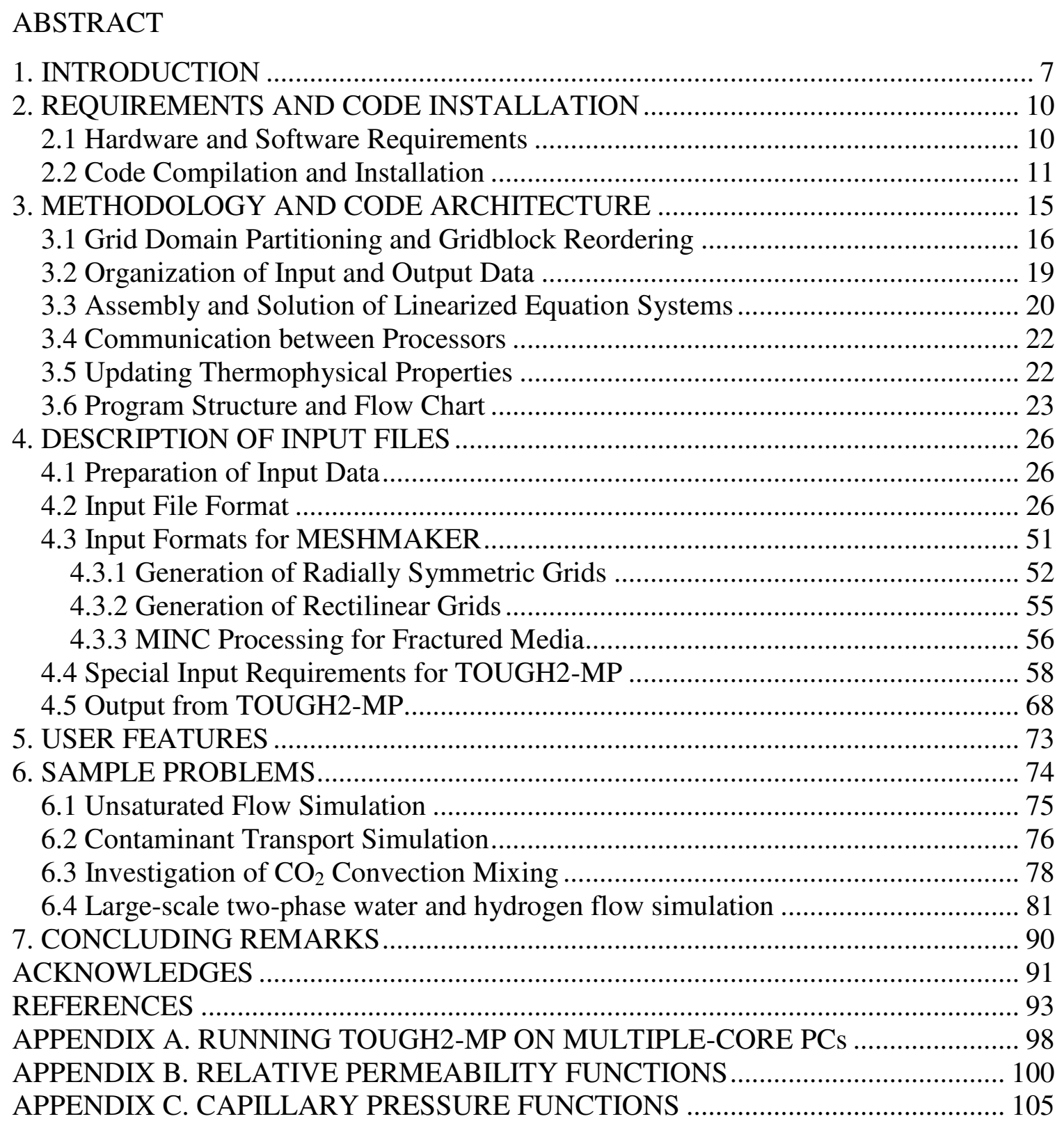




\section{INTRODUCTION}

TOUGH2 (Pruess, 1987; Pruess, 1991; Pruess et al., 1999) is a general-purpose numerical simulation program for multi-dimensional, multiphase, multicomponent fluid flows, heat transfer and contaminant transport in porous and fractured media. It has been used worldwide for geothermal reservoir engineering, nuclear waste isolation, environmental assessment and remediation, and modeling flow and transport in variably saturated media. The TOUGH2-MP code, a massive parallel version of the TOUGH2 code, was originally developed on CRAY T3E and IBM SP supercomputers (Elmroth et al., 2001; Zhang et al. 2001; Wu et al., 2002, Zhang, 2003). Since then, the parallel code has been improved in many ways by optimizing memory use, improving communication schemes, and including more fluid property modules (Zhang et al. 2003, 2006). Since its development, the parallel code has been successfully applied to large-scale simulations with up to several million gridblocks (e.g., Zhang et al., 2003a, Zhang et al. 2004, Yamamoto et al., 2007, Senger et al., 2008).

The original TOUGH2 code, an enhanced version of the TOUGH code (Pruess, 1987), was first released in 1991 (Pruess, 1991) with five basic EOS modules. The enhanced version 2.0 of the TOUGH2 code was made available to the public in 1999 and included additional fluid property modules (Pruess et al., 1999). The parallel version TOUGH2MP V1.0 (Zhang, 2003) was developed based on the original TOUGH2 V1.4 simulator (Wu et al., 1999; Wu, 1999), i.e., by implementing parallel computing algorithms into the V1.4 code. In early efforts at developing parallel simulation capabilities, Elmroth et al. (2001) developed a parallel prototype scheme for the TOUGH2 code for Massively Parallel Processor (MPP) computers. Zhang et al. (2001 and 2003) made further improvements in distributing memory requirements and improving computational efficiency for solving extremely large reservoir simulation problems with millions of gridblocks.

As compared with the previous version of the parallel code, the current version of TOUGH2-MP Version 2.0, has been significantly improved in the efficiency of its 
communication schemes. The improvements in the new version are achieved through reductions in the number of small-size messages and in the size of large messages. To achieve a faster nonlinear iteration converging speed, at each Newton iteration information exchanges across sub-domain boundaries are limited to primary variables only, while all secondary variables are updated locally, using primary variables for the sub-domain. Furthermore, the message-exchange speed is enhanced by using nonblocking communications during both linear and nonlinear iterations. We have also modified the AZTEC parallel linear-equation solver (Tuminaro et al., 1999) to nonblocking communication. All these improvements result in the current version of TOUGH2-MP being faster and more scalable than its predecessor.

In performing a parallel simulation, the TOUGH2-MP code first subdivides a simulation domain, defined by an unstructured grid of a TOUGH2 mesh, into a number of subdomains using the partitioning algorithm from the METIS software package (Karypsis and Kumar, 1998). The parallel code then relies on the MPI (Message-Passing Interface; Message Passing Forum, 1994) for its parallel implementation. Parallel simulations are run as multiple processes on a few or many processors simultaneously. Each process/processor is in charge of one portion of the simulation domain for updating thermophysical properties, assembling mass and energy balance equations, solving liner equation systems, and performing other local computations. The local linear equation systems are solved in parallel by multiple processors with the Aztec linear solver package. Although each processor solves the linearized equations of subdomains independently, the entire linear equation system is solved together by all processors collaboratively via communication between neighboring processors during each Newton iteration step.

Although TOUGH2-MP V2.0 was designed for parallel computing using multiple processors, the code can provide significant gains in computational efficiency even for single processor machines by executing nominally parallel processes in sequential mode. When multiple processors are available, it may be advantageous to partition a simulation domain into more subdomains than available processors, making the program execution partially sequential. This somewhat surprising finding can be explained from the behavior 
of the linear equation solution in the subdomains, which for large problems consumes most of the numerical work in a simulation. By partitioning into a larger number of subdomains, we obtain a larger number of smaller linear algebra problems, which can be solved more efficiently than a smaller number of larger problems. However, with increasing number of processes there also is increased overhead from message passing, which leads to optimal performance for some "intermediate" level of domain partitioning. Another advantage of running parallel processes partially sequentially is that memory requirements may be reduced, so that larger problems with more grid blocks can be tackled.

The numerical scheme of the TOUGH2 code is based on the integral finite-difference (IFD) method (Narasimhan and Witherspoon, 1976; Pruess, 1987, 1991). In the TOUGH2 formulation, conservation equations, involving mass of air, water and chemical components as well as thermal energy, are discretized in space using the IFD method. Time is discretized fully implicitly using a first-order backward finite difference scheme. The resulting discrete finite-difference equations for mass and energy balances are nonlinear and solved simultaneously using the Newton/Raphson iterative scheme. All these numerical schemes are adopted by TOUGH2-MP. The parallel code also inherits all the process capabilities of the TOUGH2 code, including descriptions of the thermodynamics and thermophysical properties of the multiphase flow system. In addition, FORTRAN 90 features are introduced to TOUGH2-MP, such as dynamic memory allocation, array operation, matrix manipulation, and replacing "common blocks" (used in the original TOUGH2) with modules. All new subroutines are written in FORTRAN 90. Program units imported from the original TOUGH2 remain in FORTRAN 77, except for the use of data modules. The current version of TOUGH2-MP includes following modules: EOS1, EOS2, EOS3, EOS4, EOS5, EOS7, EOS7R, EOS8, EOS9, ECO2N, EWASG, and T2R3D. Other members of the TOUGH family including TMVOC and TOUGH+HYDRATE (Zhang et al., 2008) have also been parallelized.

The parallelization of TOUGH2 improves modeling capabilities significantly in terms of problem size and simulation time. The code demonstrates excellent scalability. Test 
examples show that a linear or super-linear speedup can be obtained on typical Linux clusters as well as on supercomputers. Because the TOUGH2-MP parallel simulator was developed from an existing mature code, it inherits not only simulation functions from the original code, but also all other features, including input/output format, error handling, and improvements for code stability. These features provide robustness of the parallel code and ease of use for the user community of the original code, using identical input data, mesh and output files. Moreover, the domain decomposition approach and parallel computation enhance model simulation capabilities in terms of problem size and complexity to a level that cannot be reached by single-CPU codes. By using the parallel simulator, multi-million gridblock problems can be run on a typical Linux cluster with several tens to hundreds of processors to achieve ten to hundred times improvement in computational time or problem size. Our tests indicate that the parallel simulator allows much larger problems to be solved by multiple-process simulation even with a singleprocessor computer. This surprising result can be understood in terms of efficiency gains from decomposing one large linear algebra problem into a series of smaller ones, which produces super-linear speedup. The growing availability of multi-core CPUs will make parallel processing on PCs far more attractive.

This report provides a quick reference guide for utilizing the TOUGH2-MP code. The users are supposed to have basic knowledge of the original TOUGH2 family of codes. In particular, this report together with the TOUGH2 V2.0 User's Guide provides sufficient information for users to apply TOUGH2-MP to subsurface flow simulation problems. A detailed technical description of the physical processes modeled, and the mathematical and numerical methods used in the code can be found in the user's guide for TOUGH2 Version 2.0 (Pruess et al., 1999).

\section{REQUIREMENTS AND CODE INSTALLATION}

\subsection{Hardware and Software Requirements}

TOUGH2-MP has been tested on IBM and CRAY supercomputers, Linux clusters, Macs, and multi-core PCs under different operating systems. It has been successfully compiled 
using g95, and Fortran compilers from Intel, IBM, and the Portland Group. The code requires 64-bit arithmetic ( 8 byte word length for floating point numbers) for successful execution. TOUGH2-MP can be run on any shared- or distributed-memory multiple CPU computer system on which MPI is installed. The code has been run on LAM/MPI, OPEN MPI, and MPICH2.

The total computer memory required by TOUGH2-MP depends on the problem size. For a given problem, memory requirement is split among the processors used for the simulation. The code automatically distributes memory requirements to all processors based on the partitioning of the domain. All major arrays are dynamically allocated according to the numbers of local gridblocks and connections assigned by domain partitioning to each processor. As a result, larger problems can be solved using more processors on a distributed memory computer system. For example, by far the largest array used in TOUGH2-MP is "PAR", the array for storage of secondary variables. Its size in bytes (using 8-byte real data) is

$$
M=\left(N P H^{*}(N B+N K)+2\right) *(N E Q+1) * N E L * 8
$$

Here the parameters are the total number of fluid phases $N P H$, secondary parameter number $N B$, component number $N K$, and gridblock number $N E L$. If $N P H=3, N B=8$, $N K=3, N E Q=4, N E L=10^{6}$, the total memory requirement for this array is about $1400 \mathrm{MB}$. If 64 processors are used to solve this problem, each processor requires about $22 \mathrm{MB}$ of memory for this array. One of the critical bottlenecks of memory requirement is during the reading of the MESH file through the master processor. This bottleneck is avoided by a reading-distributing strategy that replaces the original MESH with two files. Detailed discussion of this approach is provided in Section 4.4.

\subsection{Code Compilation and Installation}

The source code of TOUGH2-MP consists of 10 FORTRAN files: Compu_Eos.f, Data_DD.f , Input_Output.f , Main_Comp.f , Mem_Alloc.f, Mesh_Maker.f, MULTI.f, Paral_Subs.f, TOUGH2.f, Utility_F.f , as listed in Table 2-1. Two library files 
libmetis.a and libaztec.a are also needed for compiling the parallel program. The two library files are generated by compiling the METIS and AZTEC software packages. Different EOS modules need different "Compu_Eos.f” files. Compilation of each module should use its own "Compu_Eos.f" file. In addition, the EOS9 and T2R3D modules require their own special "MULTI.f" file.

Table 2-1 List of program files of the TOUGH2-MP source code

\begin{tabular}{|l|l|l|}
\hline \multicolumn{1}{|c|}{ File name } & \multicolumn{1}{c|}{ Functions } & Note \\
\hline Main_Comp.f & $\begin{array}{l}\text { Main program for time stepping and } \\
\text { parallel running control. }\end{array}$ & Required \\
\hline Data_DD.f & Data declaration and distribution & Required \\
\hline Input_Output.f & Input and output & Required \\
\hline Compu_Eos.f & EOS Modules and satellite functions & Required \\
\hline Mem_Alloc.f & Memery allocation & Required \\
\hline Mesh_Maker.f & Meshmaker & Optional \\
\hline MULTI.f & Jacobian assembly & Required \\
\hline Para_Subs.f & Parallelization related subroutines & Required \\
\hline TOUGH2.f & Program entrance & Required \\
\hline Utility_F.f & Utility subroutines & Required \\
\hline libmetis.a & Compiled METIS functions & Library file \\
\hline libaztec.a & Compile AZTEC functions & Library file \\
\hline
\end{tabular}

Compilation and installation can be done through the following steps:

1. Download METIS at:

http://www-users.cs.umn.edu/ karypis/metis/metis/download.html

2. Compile METIS in the computer system where TOUGH2-MP will be installed.

3. Download AZTEC at: http://www.cs.sandia.gov/CRF/aztec1.html

4. Compile AZTEC in the computer system where TOUGH2-MP will be installed. (Guides for compiling METIS and AZTEC are provided with the downloaded packages.) 
5. Transfer the file "tough2-mp_2.0.tar.gz" from the installation CD-ROM to your working directory.

6. Use gunzip to unzip the file and then use the tar command to untar the archived files and directories as follows:

\section{gunzip tough2-mp_2.0.tar.gz}

\section{tar-xvf tough2-mp_2.0.tar}

A directory named tough2-mp will be created under the current working directory. Source files, make scripts, and installation test input files will be located in the subdirectories. Two additional subdirectories are created under the directory tough2mp: /tough2-mp/partition/ and /tough2-mp/utilities/.

7. Copy az_aztecf.h and libaztec.a from /aztec/lib and libmetis.a from /metis-4.0 to the subdirectory where source code is located (e.g. /tough2-mp/eos3/). libaztec.a and libmetis.a were created by successfully compiling Metis and Aztec in steps 2 and 4, respectively.

8. The "makefile" for three different compilers are provided: IBM, INTEL and PORTLAND GROUP. You can choose the one most close to your compiler. In the "makefile", a wrapper compiler, mpif90, was specified for compiling the source codes. The user may need to change the compiler name to the one installed in the computer system by editing the file "makefile" at the line containing "FC=mpif90". The user may also need to specify the path for MPI "include" and "library" files. Figure 2-1 shows a "makefile" for creating a TOUGH2-MP/EOS3 executable using PORTLAND GROUP Fortran 90.

9. Type "make" under the $\sim /$ tough2-mp/eos3/ subdirectory to compile the code. The executable file "t2eos3-mp" will be created. After compilation, type "make clean" to clean all intermediate files.

10. In order to successfully build TOUGH2-MP, the $c$ and FORTRAN compilers used for compiling the MPI system, AZTEC, METIS and TOUGH2-MP source codes must be compatible. A Fortran 90 or higher version must be used for FORTRAN source code compilation. 


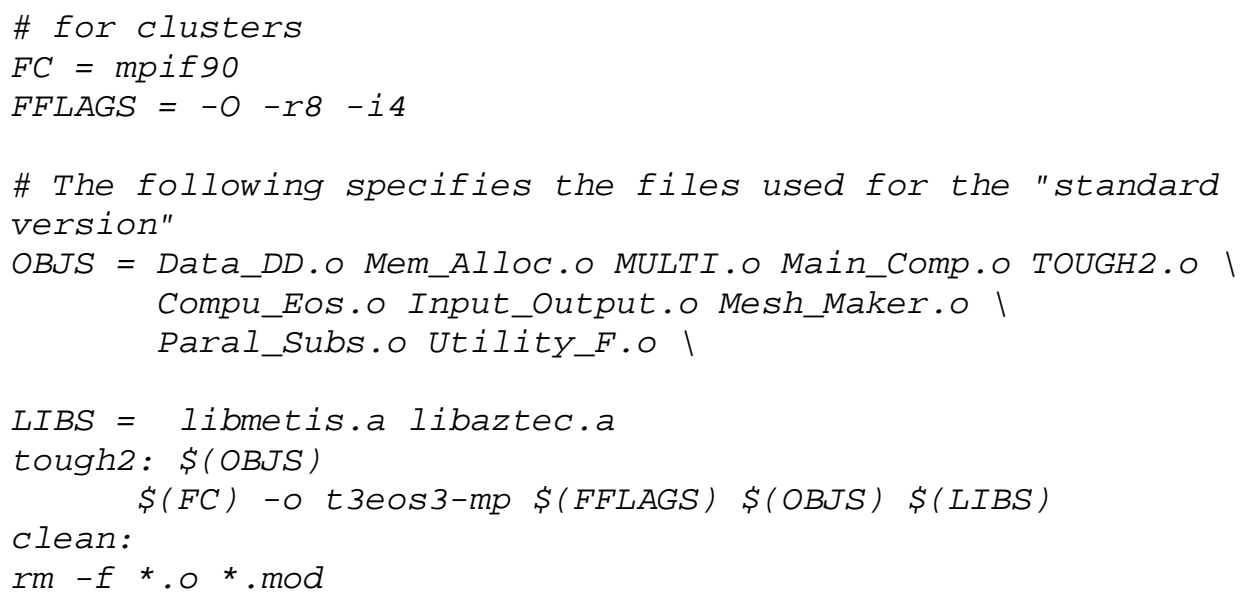

\section{Figure 2-1. A makefile for TOUGH2-MP compilation}

If "invalid communicator" or other communication problems are encountered during running the executable, user may try following:

1. Copy /tough2-mp/utilities/md_wrap_mpi_c.c to /aztec/lib to replace the original one.

2. Recompile AZTEC and then use the new library libaztec.a to recompile the TOUGH2-MP executable.

If you have difficulty using the linear solver "AZ_gmres", you may try the following:

1. Copy /tough2-mp/utilities/la_dlaic1.f to /aztec/lib to replace the original one.

2. Recompile AZTEC and then use the new library libaztec.a to recompile the TOUGH2-MP executable.

One may get additional speedup by using non-blocking communication version AZTEC by performing the following steps:

1. Copy $\sim /$ tough2-mp/utilities/az_comm.c and $\sim$ tough2-mp/utilities/az_matvec_mult.c to $\sim /$ aztec/lib to replace the original files.

2. Recompile AZTEC and then use the new library libaztec.a to recompile the TOUGH2-MP executable. 
The library file "libmetis.a" contains subroutines of the METIS package for partitioning irregular graphs and meshes. For reducing the requirement of computer memory, we use 4-byte integer for all large integer arrays in TOUGH2-MP. The corresponding arrays in METIS must also be a 4-byte integer. This can be implemented by simply removing the line of "\#define IDXTYPE_INT" in head file "struct.h" of the METIS source code. The library file "libaztec.a" provides subroutines for solving linear equation systems in parallel.

\section{METHODOLOGY AND CODE ARCHITECTURE}

Domain decomposition methods (DDM) are used as a divide and conquer strategy for solving large or time-consuming problems. The idea behind this approach is to divide the computational domain into a series of subdomains. Through the local solutions on the subdomains, a global solution is formed. Solutions for subdomains can be sought simultaneously. Therefore this approach is suitable for parallel computations as long as the computational work can be evenly distributed. The TOUGH2-MP numerical computational scheme is based on a fully implicit formulation with Newton iteration. The resulting linearized equations are solved by a parallel linear solver from the AZTEC package (Tuminaro et al., 1999). AZTEC includes a number of Krylov iterative methods, such as conjugate gradient (CG), generalized minimum residual (GMRES) and stabilized biconjugate gradient (BiCGSTAB). Fully implicit scheme has been proven to be the most robust numerical approach in modeling multiphase flow and heat transfer in reservoirs over the past several decades. For a typical simulation with the fully implicit scheme and Newton iteration, such as in the TOUGH2 run, the most time-consuming steps of the execution consist of three parts: (1) updating thermophysical parameters, (2) assembling the Jacobian matrix, and (3) solving the linearized system of equations. Consequently, one of the most important aims of a parallel simulation is to distribute computational time for these three parts. In addition, a parallel scheme must take into account domain decomposition, grid node/element reordering, data input and output optimizing, and efficient message exchange between processors. These important parallel-computing strategies and implementation procedures are discussed below. 


\subsection{Grid Domain Partitioning and Gridblock Reordering}

Developing an efficient and effective method for partitioning unstructured grid domains is a critical step for a successful parallel-computing scheme. Firstly, to achieve better numerical performance, parallel simulations require the distribution of gridblocks evenly to different processing elements (PEs) or processors, i.e., the number of gridblocks assigned to each PE should be roughly the same. Secondly, the number of connections across domain bounds is minimized. The goal of the first requirement is to balance computational work among the processors. The goal of the second requirement is to minimize the time consumed in communication between processors (resulting from the estimation of the coupling terms or connections across the domain bounds by different processors).

In a TOUGH2-MP simulation, a model domain, or grid, is represented by a set of one-, two- or three-dimensional gridblocks (elements), and the interfaces between any two gridblocks are represented by connections. The entire grid system is treated as an unstructured grid. From the connection information, an adjacency matrix can be constructed. The adjacency or connection structure of the model meshes is stored in a compressed storage format (CSR).

The adjacency structure of storing the model grid can be described as follows: In the CSR format, the adjacency structure of a global-mesh domain with $n$ gridblocks and $m$ connections is represented by two arrays, xadj and $a d j$. The $x a d j$ array has a size of $n+1$, whereas the $a d j$ array has a size of $2 m$. Assuming that element numbering starts from 1 , the adjacency list of element $i$ is stored in an array $\operatorname{adj}$, starting at index $\operatorname{xadj}(i)$ and ending at index $\operatorname{xadj}(i+1)-1$. That is, for each element $i$, its adjacency list is stored in the consecutive locations in the array $a d j$, and the array $x a d j$ is used to point to where it begins and where it ends. Figure 3-1a shows the connection of a 12-element domain; Figure 3-1b illustrates its corresponding CSR-format arrays.

We utilize one of the three partitioning algorithms provided by the METIS software package (version 4.0) (Karypsis and Kumar, 1998) for the grid domain partitioning. The 
three algorithms are denoted, respectively, as the $K$-way, the $V K$-way, and the Recursive partitioning algorithm. $K$-way is used for partitioning a global mesh (graph) into a large number of partitions (more than 8). The objective of this algorithm is to minimize the number of edges that straddle different partitions. If a small number of partitions is desired, the Recursive partitioning method, a recursive bisection algorithm, should be used. $V K$-way is a modification to $K$-way and its objective is to minimize the total communication volume. Both $K$-way and $V K$-way belong to multilevel partitioning algorithms.

Figure 3-1a shows a scheme for partitioning a sample domain into three parts. Gridblocks are assigned to different processors through partitioning methods and reordered by each processor to a local index ordering. Elements corresponding to these blocks are explicitly stored in the processor and are defined by a set of indices referred to as the processor's update set. The update set is further divided into two subsets: internal and border. Elements of the internal set are updated using only the information on the current processor. The border set consists of blocks with at least one edge to a block assigned to another processor. The border set includes blocks that would require values from the other processors to be updated. The set of blocks that are not in the current processor, but needed to update the components in the border set, is referred to as an external set. Table 3-1 shows the partitioning results. One of the local numbering schemes for the sample problem is presented in Figure 3-1a.

The local numbering of gridblocks is carried out to facilitate the communication between processors. The numbering sequence is internal block set followed by border block set and finally by the external block set. In addition, all external blocks on the same processor are in a consecutive order. 


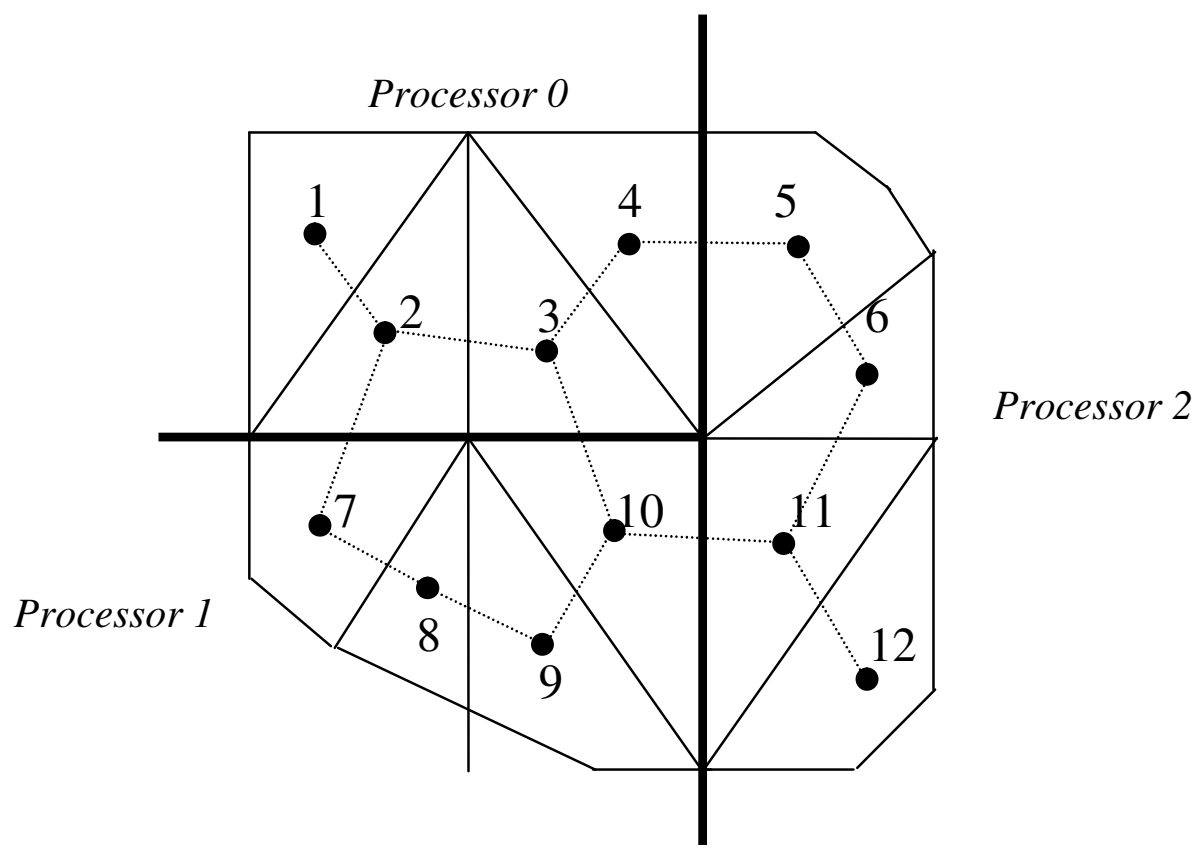

(a) A 12-elements domain partitioning on 3 processors

\begin{tabular}{l|l|l|l|l|l|l|l|l|l|l|l|l|l} 
Elements & 1 & 2 & 3 & 4 & 5 & 6 & 7 & 8 & 9 & 10 & 11 & 12 \\
\hline xadj & 1 & 2 & 5 & 8 & 10 & 12 & 14 & 16 & 18 & 20 & 23 & 26 & 27 \\
\hline adj & & 2 & $1,3,7$ & $2,4,10$ & 3,5 & 4,6 & 5,11 & 2,8 & 7,9 & 8,10 & $3,9,11$ & $6,10,12$ & 11
\end{tabular}

(b) CSR format

Figure 3-1 An example of domain partitioning and CSR format for storing connections

Table 3-1. Example of Domain Partitioning and Local Numbering

\begin{tabular}{|c|c|c|c|c|}
\hline & \multicolumn{2}{|c|}{ Update } & \multirow[t]{2}{*}{ External } \\
\hline & & Internal & Border & \\
\hline \multirow[t]{2}{*}{ Processor 0} & Gridblocks & 1 & $\begin{array}{lll}2 & 3 & 4\end{array}$ & 5710 \\
\hline & Local Numbering & 1 & $\begin{array}{lll}2 & 3 & 4\end{array}$ & $\begin{array}{lll}5 & 6 & 7\end{array}$ \\
\hline \multirow[t]{2}{*}{ Processor 1} & Gridblocks & $\begin{array}{ll}8 & 9\end{array}$ & $\begin{array}{ll}7 & 10\end{array}$ & $\begin{array}{lll}2 & 3 & 11\end{array}$ \\
\hline & Local Numbering & & 3 & 67 \\
\hline \multirow[t]{2}{*}{ Processor 2} & Gridblocks & $\begin{array}{ll}6 & 12\end{array}$ & $\begin{array}{ll}5 & 11\end{array}$ & 410 \\
\hline & Local Numbering & & 3 & 5 \\
\hline
\end{tabular}


Only nonzero entries of a submatrix for a partitioned mesh domain are stored on each processor. Each processor stores only the rows that correspond to its update set (including internal and border blocks, See Table 3-1). These rows form a submatrix whose entries correspond to the variables of both the update set and the external set defined on this processor.

\subsection{Organization of Input and Output Data}

The input data of TOUGH2-MP include hydrogeologic parameters and constitutive relations of porous media and fluids, such as absolute and relative permeability, porosity, capillary pressure, thermophysical properties of fluids and rock, and initial and boundary conditions of the system. Other processing requirements include the specification of space-discretized geometric information (grid) and various program options (computational parameters and time-stepping information). For a large-scale, threedimensional model, a computer memory of several gigabytes is generally required and the distribution of the memory to all processors is necessary for practical application of TOUGH2-MP.

To efficiently use the memory of each processor (considering that each processor has a limited memory available), the input data files for the TOUGH2-MP simulation are organized in sequential format. There are two large groups of data blocks within a TOUGH2-MP mesh file: one with dimensions equal to the number of gridblocks; the other with dimensions equal to the number of connections (interfaces). Large data blocks are read one by one through a temporary full-sized array and then distributed to different processors. This method avoids storing all input data in a single processor (whose memory space may be too small) and greatly enhances the I/O efficiency. Other smallvolume data, such as simulation control parameters, are duplicated onto all processors.

All data input and output are carried out through the master processor. For extremely large-scale problems, outputs may be performed by all processors involved in the computation with multiple files by each processor writing out its own portion simulation results. This approach may avoid extensive communication for output. Time series 
outputs are written out by processors at which the specified elements or connections for output are located. This approach could be extremely efficient for high latency computer systems.

\subsection{Assembly and Solution of Linearized Equation Systems}

In the TOUGH2-MP formulation, the discretization in space using the IFD leads to a set of strongly coupled nonlinear algebraic equations, which are linearized by the Newton method. Within each Newton iteration step, the Jacobian matrix is first constructed by numerical differentiation. The resulting system of linear equations is then solved using an iterative linear solver with different preconditioning procedures. The following gives a brief discussion of assembling and solving the linearized equation systems with parallel simulation.

The discrete mass and energy balance equations solved by the TOUGH2 code can be written in a residual form (Pruess, 1991; Pruess et al., 1999):

$R_{n}^{\kappa}\left(x^{t+1}\right)=M_{n}^{\kappa}\left(x^{t+1}\right)-M_{n}^{\kappa}\left(x^{t}\right)-\frac{\Delta t}{V_{n}}\left\{\sum_{m} A_{n m} F_{n m}^{\kappa}\left(x^{t+1}\right)+V_{n} q_{n}^{\kappa, t+1}\right\}=0$

where the vector $x^{t}$ consists of primary variables at time $t, R_{n}^{\kappa}$ is the residual of component $\kappa$ (heat is regarded as a "component") for block $n, M$ denotes mass or thermal energy per unit volume for component $\kappa, V_{n}$ is the volume of the block $n$, and $q$ denotes sinks and sources of mass or energy, $\Delta t$ denotes the current time step size, $t+1$ denotes the current time, $A_{n m}$ is the interface area between blocks $n$ and $m$, and $F_{n m}$ is the "flow" term of mass or energy exchange between blocks $n$ and $m$.

Equation (3.1) is solved using the Newton method, leading to

$$
-\left.\sum_{i} \frac{\partial R_{n}^{\kappa, t+1}}{\partial x_{i}}\right|_{p}\left(x_{i, p+1}-x_{i, p}\right)=R_{n}^{\kappa, t+1}\left(x_{i, p}\right)
$$


where $x_{i, p}$ represents the value of $i^{\text {th }}$ primary variable at the $p^{\text {th }}$ iteration step.

The Jacobian matrix as well as the right-hand side of (3.2) needs to be recalculated at each Newton iteration, such that computational efforts may be extensive for a large simulation. In the parallel code, the assembly of the linear equation system (3.2) is shared by all processors, and each processor is responsible for computing the rows of the Jacobian matrix that correspond specifically to the blocks in the processor's own update set. Computation of the elements in the Jacobian matrix is performed in two parts. The first part consists of the computations related to the individual blocks (accumulation and source/sink terms). Such calculations are carried out using the information stored on the current processor, without need of communication with other processors. The second part includes all the computations related to the connections or flow terms. Elements in the border set need information from the external set, which requires communication with neighboring processors. Before performing these computations, an exchange of relevant primary and updating secondary variables are required. For the elements corresponding to border set blocks, each processor sends these elements to the different but related processors, which receive these elements as external blocks.

The Jacobian matrix for local gridblocks in each processor is stored in the distributed variable block row (DVBR) format, a generalization of the VBR format. All matrix blocks are stored row-wise, with the diagonal blocks stored first in each block row. Scalar elements of each matrix block are stored in column major order. The data structure consists of a real-type vector and five integer-type vectors, forming the Jacobian matrix. Detailed explanation of the DVBR data format can be found in Tuminaro et al. (1999).

The linearized equation system arising at each Newton step is solved using an iterative linear solver from the AZTEC package. There are several different solvers and preconditioners from the package for users to select and the options include conjugate gradient, restarted generalized minimal residual, conjugate gradient squared, transposedfree quasi-minimal residual, and bi-conjugate gradient with stabilization methods. The work for solving the global linearized equation is shared by all processors, with each 
processor responsible for computing its own portion of the partitioned domain equations. To accomplish the parallel solution, communication between a pair of processors is required to exchange data between the neighboring grid partitions. Moreover, global communication is also required to compute the norms of vectors for checking the convergence.

During a parallel simulation, the time-step size is automatically adjusted (increased or reduced), depending on the convergence rate of iterations. In the TOUGH2-MP code, time-step size is calculated at the first processor (master processor, named PE0) after collecting necessary data from all processors. The convergence rates may be different in different processors. Only when all processors reach stopping criteria will the time march to the next time step.

\subsection{Communication between Processors}

Communication between processors working on neighboring/connected gridblocks, partitioned into different domains, is an essential component of the parallel algorithm. Moreover, global communication is also required to compute norms of vectors, contributed by all processors, for checking the convergence. In addition to the communication taking place inside the linear solver routine to solve the linear equation system, communication between neighboring processors is necessary to update primary variables. A subroutine is used to manage data exchange between processors. When the subroutine is called by a processor, an exchange of vector elements corresponding to the external set of the gridblocks is performed. During time stepping or Newton iteration, exchange of external variables is required for the vectors containing the primary variables. More discussion on the prototype scheme used for data exchange is given in Elmroth et al. (2001). In addition, we have further improved the schemes by introducing non-blocking communication to the Aztec package and Newton iterations (Zhang and $\mathrm{Wu}, 2006$ )

\subsection{Updating Thermophysical Properties}

The thermophysical properties of fluid mixtures (secondary variables) needed for assembling the governing mass- and energy-balance equations are calculated at the end of 
each Newton iteration step based on the updated set of primary parameters. In the same time, the phase conditions are identified for all gridblocks, the appearance or disappearance of phase is recognized, and primary variables are switched and properly re-initialized in response to a change of phase. All these tasks must be done gridblock by gridblock for the entire simulation domain. The computational work for these tasks is readily parallelized by each processor handling its corresponding subdomain. A tiny overlapping of computation is needed for the gridblocks at the neighboring subdomain border to avoid communication for secondary variables.

\subsection{Program Structure and Flow Chart}

TOUGH2-MP has a program structure very similar to the original version of TOUGH2, except that the parallel version solves a problem using multiple processors. We implement dynamic memory allocation, modules, array operations, matrix manipulation, and other FORTRAN 90 features in the parallel code. In particular, the message-passing interface (MPI) library of Message Passing Forum (1994) is used for message passing. Another important modification to the original code is in the time-step looping subroutine. This subroutine now provides the general control of problem initialization, grid partitioning, data distribution, memory requirement balancing among all processors, time stepping, and output options.

In summary, all data input and output are carried out through the master processor. The most time-consuming computations (assembling the Jacobian matrix, updating thermophysical parameters, solving linear equation systems.) are distributed to all processors involved. The memory requirements are also distributed to all processors. Distributing both computing and memory requirements is essential for solving large-scale problems and obtaining better parallel performance. Figure 3-2 gives an abbreviated overview of the program flow chart. 


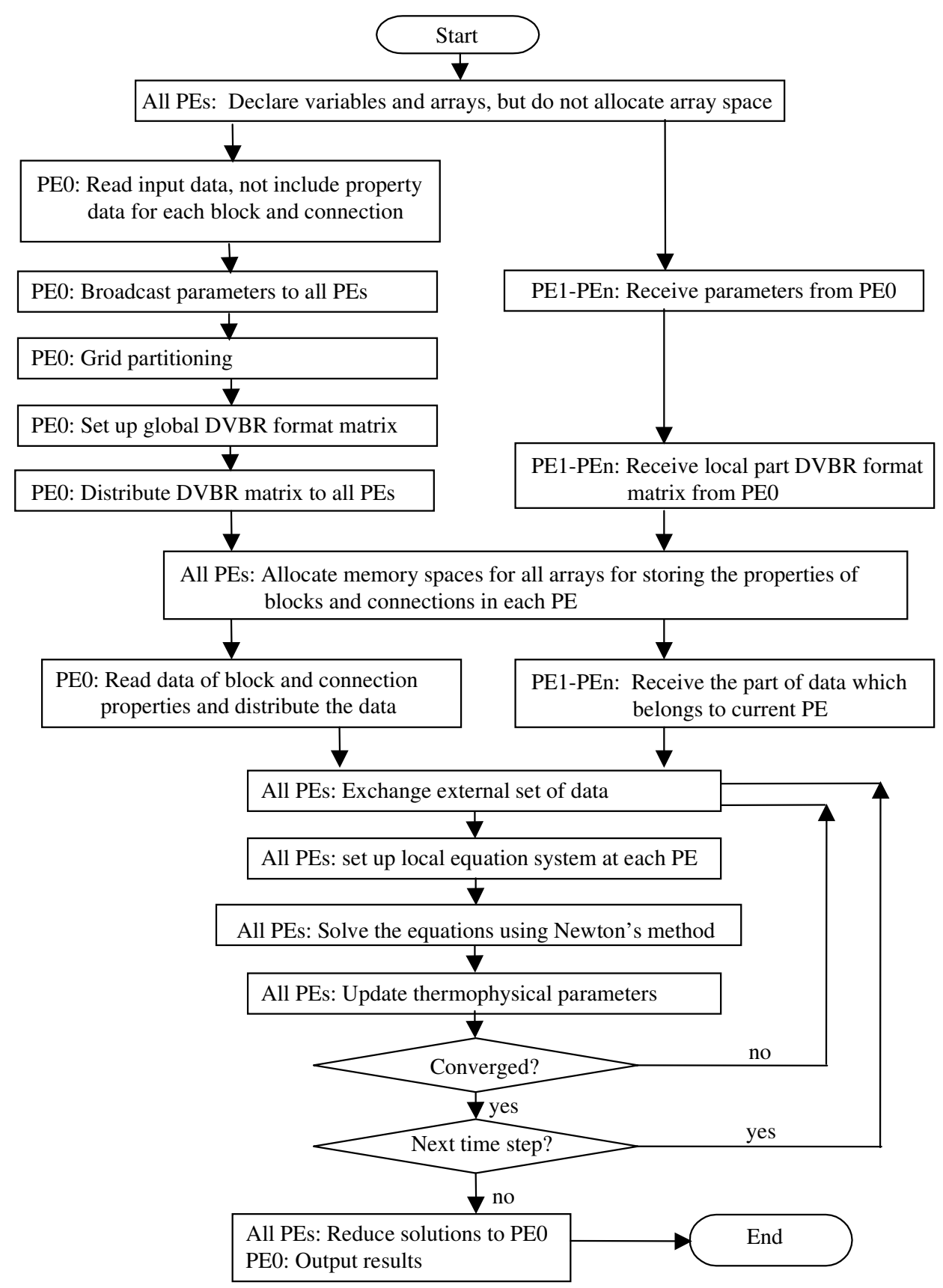

Figure 3-2. Simplified flow chart of TOUGH2-MP 
Table 4-1. TOUGH2-MP input data blocks ${ }^{\S}$

\begin{tabular}{|c|c|}
\hline Keyword & Function \\
\hline $\begin{array}{l}\text { TITLE } \\
\text { (first record) }\end{array}$ & One data record (single line) with a title for the simulation problem \\
\hline VER14 & Optional; invoke using the Version 1.4 processing features. \\
\hline MESHM & Optional; parameters for internal grid generation through MESHMaker \\
\hline ROCKS & Hydrogeologic parameters for various reservoir domains \\
\hline MULTI & $\begin{array}{l}\text { Optional; specifies number of fluid components and balance equations } \\
\text { per gridblock; applicable only for certain fluid property (EOS) modules }\end{array}$ \\
\hline START & Optional; one data record for more flexible initialization \\
\hline PARAM & Computational parameters. \\
\hline RPCAP & $\begin{array}{l}\text { Optional; parameters for relative permeability and capillary pressure } \\
\text { functions }\end{array}$ \\
\hline TIMES & Optional; specification of times for generating printout \\
\hline *ELEME & List of gridblocks (volume elements) \\
\hline$*$ CONNE & List of flow connections between gridblocks \\
\hline *GENER & Optional; list of mass or heat sinks and sources \\
\hline INDOM & Optional; list of initial conditions for specific reservoir domains \\
\hline$*$ INCON & Optional; list of initial conditions for specific gridblocks \\
\hline $\begin{array}{l}\text { NOVER } \\
\text { (optional) }\end{array}$ & $\begin{array}{l}\text { Optional; if present, suppresses printout of version numbers and dates of } \\
\text { the program units executed in a TOUGH } 2 \text { run }\end{array}$ \\
\hline TIMBC & Optional; introducing a table for time-dependent pressure boundary. \\
\hline RTSOL & Optional; provide linear solver parameters \\
\hline FOFT & Optional; list of gridblocks for time-dependent output \\
\hline GOFT & Optional; list of source/sink gridblocks for time-dependent output. \\
\hline COFT & Optional; list of connections for time-dependent output \\
\hline DIFFU & Optional; introduce diffusion coefficients \\
\hline SELEC & Optional, provide parameters for requirements by specific modules \\
\hline $\begin{array}{l}\text { ENDCY } \\
\text { (last record) }\end{array}$ & One record to close the TOUGH2 input file and initiate the simulation \\
\hline ENDFI & $\begin{array}{l}\text { Alternative to "ENDCY" for closing a TOUGH } 2 \text { input file; will cause } \\
\text { flow simulation to be skipped; useful if only mesh generation is desired }\end{array}$ \\
\hline
\end{tabular}

$\S$ Blocks labeled with a star * can be provided as separate disk files, in which case they would be omitted from the INFILE file. 


\section{DESCRIPTION OF INPUT FILES}

\subsection{Preparation of Input Data}

Input of TOUGH2-MP is provided through a file named INFILE or separate additional files (e.g. MESH, GENER, INCON), organized into a number of data blocks, labeled by five-character keywords (Table 4-1). The input file "INFILE" of TOUGH2-MP is compatible with the input file for TOUGH2 V1.4 and T2R3D V1.4 (Wu, 1999 and 2000), and also the TOUGH2 V2.0. The parallel program may also receive additional data input through optional input files (See Section 4.4 for details). In general, input files for V1.4 and 2.0 or combination of both are readily acceptable for the parallel simulator.

\subsection{Input File Format}

This section presents the data input formats for TOUGH2-MP. Most formats are identical to corresponding inputs in V1.4 and V2.0. Please refer to the TOUGH2 User's Guide Version 2.0 (Pruess et al., 1999, Wu et. al., 1996), and User's Manual for TOUGH2 V1.4 and T2R3D V1.4 (Wu, 1999 and 2000) for more information.

TITLE is the first record of the input file, containing a header of up to 80 characters, to be printed on the output. This can be used to identify a problem. If no title is desired, leave this record blank.

VER14 the default version of the parallel code is compatible with TOUGH2 V2.0. Some modules (EOS3, EOS9, T2R3D) can be run with both V1.4 or V2.0 (V1.4 has its own specific features). To use V1.4, this keyword must be presented right after the line for TITLE keyword.

MESHM introduces parameters for internal mesh generation and processing. The MESHMaker input has a modular structure organized by keywords. Detailed instructions for preparing MESHMaker input are given in Section 4.3. 
$\underline{\text { Record MESHM.1 }}$

Format(A5)

WORD

WORD Enter one of several keywords, such as RZ2D, RZ2DL, XYZ, MINC, to generate different kinds of computational meshes.

Record MESHM.2 A blank record closes the MESHM data block.

ENDFI is a keyword that can be used to close a TOUGH2-MP input file when no flow simulation is desired. This will often be used for a mesh generation run when some hand-editing of the mesh will be needed before the actual flow simulation.

ROCKS introduces material parameters for different reservoir domains.

Record ROCKS.1

Format (A5, I5, 7E10.4)

MAT, NAD, DROK, POR, (PER (I), I = 1,3), CWET, SPHT

MAT Material name (rock type).

NAD If zero or negative, defaults will take effect for a number of parameters (see below);

$\geq 1$ : will read another data record to override defaults.

$\geq 2$ : will read two more records with domain-specific parameters for relative permeability and capillary pressure functions.

DROK Rock grain density $\left(\mathrm{kg} / \mathrm{m}^{3}\right)$

POR Default porosity (void fraction) for all elements belonging to domain "MAT" for which no other porosity has been specified in block INCON. Option "START" is necessary for using default porosity.

PER(I), $\quad \mathrm{I}=1,3$ absolute permeabilities along the three principal axes, as specified by ISOT in block CONNE. 
CWET Formation heat conductivity under fully liquid-saturated conditions $\left(\mathrm{W} / \mathrm{m}{ }^{\circ} \mathrm{C}\right)$.

SPHT Rock grain specific heat $\left(\mathrm{J} / \mathrm{kg}{ }^{\circ} \mathrm{C}\right)$. Domains with SPHT $>10^{4} \mathrm{~J} / \mathrm{kg}$ ${ }^{\circ} \mathrm{C}$ will not be included in global material balances. This provision is useful for boundary nodes, which are given very large volumes so that their thermo-dynamic state remains constant. Because of the large volume, inclusion of such nodes in global material balances would make the balances useless.

$\underline{\text { Record ROCKS.1.1 (optional, NAD } \geq 1 \text { only) }}$

Format (8E10.4)

COM, EXPAN, CDRY, TORTX, GK, PERF(1)/XKD3, PERF(2)/XKD4, PERF(3)

COM Pore compressiblity $\left(\mathrm{Pa}^{-1}\right),(1 / \phi)(\partial \phi / \partial \mathrm{P})_{\mathrm{T}}$ (default is 0$)$.

EXPAN Pore expansivity $\left(1 /{ }^{\circ} \mathrm{C}\right),(1 / \phi)(\partial \phi / \partial \mathrm{T})_{\mathrm{P}}$ (default is 0$)$.

CDRY Formation heat conductivity under desaturated conditions (W/m $\left.{ }^{\circ} \mathrm{C}\right)$, (default is CWET).

TORTX Tortuosity factor for binary diffusion.

GK Klinkenberg parameter $\mathrm{b}\left(\mathrm{Pa}^{-1}\right)$ for enhancing gas phase permeability according to the relationship $\mathrm{k}_{\mathrm{gas}}=\mathrm{k}_{\mathrm{liq}} *(1+\mathrm{b} / \mathrm{P})$.

The following three slots are for different parameters in Version 1.4 and 2.0.

For Ver 1.4:

PERF(1) Absolute fracture continuum permeabilities along one principal axis, as specified by ISOT $=1$ in block CONNE, for using the ECM only.

PERF(2) Absolute fracture continuum permeabilities along one principal axis, as specified by ISOT $=2$ in block CONNE, for using the ECM only.

PERF(3) Absolute fracture continuum permeabilities along one principal axis, as specified by ISOT $=3$ in block CONNE, for using the ECM only. 
For a dual-continuum model of dual-permeability, double-porosity or MINC, $\operatorname{PERF}(3)$ is effective porosity of fracture continuum and in this case, $\operatorname{PERF}(1)$ and $\operatorname{PERF}(2)$ must be set to zero.

For Ver 2.0:

XKD3 Distribution coefficient for parent radionuclide, Component 3, in the aqueous phase, $\mathrm{m}^{3} / \mathrm{kg}$ (EOS7R only).

XKD4 Distribution coefficient for daughter radionuclide, Component 4, in the aqueous phase, $\mathrm{m}^{3} / \mathrm{kg}$ (EOS7R only).

$\underline{\text { Record ROCKS.1.2 }}$ (optional, NAD $\geq 2$ only)

Format (I5, 5X,7E10.4)

$\mathrm{IRP},(\mathrm{RP}(\mathrm{I}), \mathrm{I}=1,7)$

IRP Integer parameter to choose type of relative permeability function (see Appendix B).

$\mathrm{RP}(\mathrm{I}), \mathrm{I}=1, \ldots, 7$ parameters for relative permeability function (Appendix B).

$\underline{\text { Record ROCKS.1.3 }}$ (optional, NAD $\geq 2$ only)

Format (I5, 5X,7E10.4)

$\mathrm{ICP},(\mathrm{CP}(\mathrm{I}), \mathrm{I}=1,7)$

ICP Integer parameter to choose type of capillary pressure function (see Appendix B).

$\mathrm{CP}(\mathrm{I}) \quad \mathrm{I}=1, \ldots, 7$ parameters for capillary pressure function (Appendix $\mathrm{C}$ ). Repeat records 1, 1.1, 1.2, and 1.3 for any number of reservoir domains.

For T2R3D, an additional rock card is needed for radionuclide transport properties, which should be located right before the ROCKS.1.2

Record ROCKS.1.1.5 (For T2R3D only)

FORMAT(6E10.4)

ALPHAL, ALPHAT, ALAMDA, SKD, DIFFM, ALPHAFM

ALPHAL longitudinal dispersivity (m)

ALPHAT transverse dispersivity (m)

ALAMDA radioactive decay constant $=\ln (2) / t_{1 / 2}(1 / s)$ 
SKD distribution coefficient, $\mathrm{K}_{\mathrm{d}}\left(\mathrm{m}^{3} / \mathrm{kg}\right)$

DIFFM molecular diffusion coefficient in liquid phase $\left(\mathrm{m}^{2} / \mathrm{s}\right)$

ALPHAFM averaged dispersivity for fracture/matrix (m)

Record ROCKS.2 A blank record closes the ROCKS data block.

MULTI Permits the user to select the number and nature of balance equations that will be solved. The keyword MULTI is followed by a single data record. For most EOS modules this data block is not needed, as default values are provided internally. Available parameter choices are different for different EOS modules.

$\underline{\text { Record MULTI. } 1}$

Format (5I5)

NK, NEQ, NPH, NB, NKIN

NK Number of mass components.

NEQ number of balance equations per grid block. Usually we have NEQ $=\mathrm{NK}+1$, for solving NK mass and one energy balance equation. Some EOS modules allow the option NEQ $=$ NK, in which case only NK mass balances and no energy equation will be solved.

NPH Number of phases that can be present (2 for mostt modules).

NB Number of secondary parameters in the PAR-array (see Fig. 3) other than component mass fractions. Available options include $\mathrm{NB}=6$ (no diffusion) and $\mathrm{NB}=8$ (include diffusion). It always equal 8 for Ver 1.4.

NKIN Number of mass components in INCON data (default is NKIN = NK). This parameter can be used, for example, to initialize an EOS7R simulation $(\mathrm{NK}=4$ or 5$)$ from data generated by EOS7 ( $\mathrm{NK}=2$ or 3 ). If a value other than the default is to be used, then data block MULTI must appear before any initial conditions in data blocks PARAM, INDOM, or INCON. 
START (optional)

A record with START typed in columns 1-5 allows a more flexible initialization. More specifically, when START is present, INCON data can be in arbitrary order, and need not be present for all gridblocks (in which case defaults will be used). Without START, there must be a one-to-one correspondence between the data in blocks ELEME and INCON.

PARAM introduces computation parameters, time stepping information, and default initial conditions.

$\underline{\text { Record PARAM.1 }}$

Format (2I2, 3I4, 24I1, 10X, 2E10.4, I10).

NOITE, KDATA, MCYC, MSEC, MCYPR, (MOP(I), I = 1, 24), TEXP, BE, MCYCF, MOP(25)

NOITE Specifies the maximum number of Newtonian iterations per time step (default is 8)

KDATA Specifies amount of printout (default is 1).

$=0$ or 1: print a selection of the most important variables.

$=2:$ in addition, print mass and heat fluxes and flow velocities.

$=3:$ in addition, print primary variables and their changes.

MCYC Maximum number of time steps to be calculated.

MSEC Maximum duration, in CPU seconds, of the simulation

(default is infinite).

MCYPR Printout will occur for every multiple of MCYPR steps (default is 1).

$\operatorname{MOP}(\mathrm{I}), \mathrm{I}=1,24$ allows choice of various options, which are documented in printed output from a TOUGH2 run.

MOP(1) If unequal 0, a short printout for nonconvergent iterations will be generated.

MOP(2) through MOP(6) generate additional printout in various subroutines, if set unequal 0 . This feature should not be needed in 
normal applications, but it will be convenient when a user suspects a bug and wishes to examine the inner workings of the code. The amount of printout increases with MOP(I) (consult source code listings for details).

MOP(2) CYCIT (main subroutine).

MOP(3) MULTI (flow and accumulation terms).

MOP(4) QU (sinks/sources).

MOP(5) EOS (equation of state).

MOP(6) LINEQ (linear equations).

$\operatorname{MOP}(7) \quad$ If unequal 0 , a printout of input data will be provided.

Calculation option choices are as follows:

MOP(9) Determines the composition of produced fluid with the MASS option (see GENER, below). The relative amounts of phases are determined as follows:

$=0$ : according to relative mobility in the source element.

$=1$ : produced source fluid has the same phase composition as the producing element.

MOP(10) Chooses the interpolation formula for heat conductivity of rock as a function of liquid saturation $\left(\mathrm{S}_{1}\right)$

$$
\begin{array}{ll}
=0: & \mathrm{C}\left(\mathrm{S}_{1}\right)=\mathrm{CDRY}+\mathrm{SQRT}\left(\mathrm{S}_{1} *[\mathrm{CWET}-\mathrm{CDRY}]\right) \\
=1: & \mathrm{C}\left(\mathrm{S}_{1}\right)=\mathrm{CDRY}+\mathrm{S}_{1} *(\mathrm{CWET}-\mathrm{CDRY}) \\
=2: & \mathrm{C}=\mathrm{C} 0+\mathrm{C} 1 * \mathrm{~T}+\mathrm{C} 2 * \mathrm{~S}_{1}+\mathrm{C} 3 * \mathrm{POR} .
\end{array}
$$

MOP(11) Determines evaluation of mobility and permeability at interfaces.

=0: mobilities are upstream weighted with WUP (see PARAM.3), permeability is upstream weighted.

=1: mobilities are averaged between adjacent elements, permeability is upstream weighted.

$=2$ : $\quad$ mobilities are upstream weighted, permeability is harmonic weighted.

=3: mobilities are averaged between adjacent elements, permeability is harmonic weighted. 
= 4: mobility and permeability are both harmonic weighted.

MOP(12) Determines interpolation procedure for time dependent sink/source data (flow rates and enthalpies).

$=0: \quad$ triple linear interpolation; tabular data are used to obtain interpolated rates and enthalpies for the beginning and end of the time step; the average of these values is then used.

=1: step function option; rates and enthalpies are taken as averages of the table values corresponding to the beginning and end of the time step.

$=2$ : $\quad$ rigorous step rate capability for time dependent generation data. A set of times $t_{i}$ and generation rates $q_{i}$ provided in data block GENER is interpreted to mean that sink/source rates are piecewise constant and change in discontinuous fashion at table points.

MOP(14) Specifies if 5- or 8-character elements are used in the mesh.

$=0: \quad 5$-character elements are used.

=1: 8-character elements are used.

MOP(15) Determines conductive heat exchange with impermeable confining layers

$=0$ : heat exchange is off.

$=1$ : heat exchange is on (for gridblocks that have a non-zero heat transfer area; see data block ELEME). This option has not been implemented in TOUGH2-MP.

MOP(16) Provides automatic time step control. Time step size will be increased if convergence occurs within ITER $\leq$ MOP(16) NewtonRaphson iterations. It is recommended to set $\mathrm{MOP}(16)$ in the range of 2 - 4 .

MOP(17) Specifies generation of a flow9.dat file for T2R3D transport simulations (EOS9 only).

$=0:$ no.

$=1: \quad$ yes. 
MOP(18) Selects handling of interface density.

$=0$ : perform upstream weighting for interface density.

$>0$ : average interface density between the two gridblocks.

However, when one of the two phase saturations is zero, upstream weighting will be performed.

MOP(19) Switch used by different EOS modules for conversion of primary variables.

MOP(20) Allows for different formats of CONNE and GENER indexes.

$=0: \quad$ use format $(16 I 5)$.

$=1: \quad$ use format $(10 \mathrm{I} 8)$

$\operatorname{MOP}(21) \quad$ Allows for one more N/R iteration after solution.

$=0: \quad$ no need for one more iteration.

$=1$ : perform one more iteration after convergence.

MOP(24) Determines handling of multiphase diffusive fluxes at interfaces.

$=0$ : $\quad$ harmonic weighting of fully coupled effective multiphase diffusivity.

=1: $\quad$ separate harmonic weighting of gas and liquid phase diffusivities.

TEXP Parameter for temperature dependence of gas phase diffusion coefficient.

BE (Optional) parameter for effective strength of enhanced vapor diffusion; if set to a non-zero value, will replace the parameter group $\phi \tau_{0} \tau_{\beta}$ for vapor diffusion.

MCYCF Allows for more time steps to be run for each simulation by $\mathrm{MCYC}=\mathrm{MCYCF}$, if MCYCF $>\mathrm{MCYC}$.

Record PARAM.2

Format (4E10.4, A5, 5X,3E10.4)

TSTART, TIMAX, DELTEN, DELTMX, ELST, GF, REDLT, SCALE

TSTART Starting time of simulation in seconds (default is 0 ). 
TIMAX Time in seconds at which simulation should stop (default is infinite).

DELTEN Length of time steps in seconds. If DELTEN is a negative integer, DELTEN $=$-NDLT, the program will proceed to read NDLT records with time step information. Note that -NDLT must be provided as a floating point number, with decimal point.

DELTMX Upper limit for time step size in seconds (default is infinite)

ELST Writes a file for time versus primary variables for selected elements at all the times, when ELST = RICKA (Same as the function with keyword FOFT).

GF Magnitude $\left(\mathrm{m} / \mathrm{sec}^{2}\right)$ of the gravitational acceleration vector.

Blank or zero gives "no gravity" calculation.

REDLT Factor by which time step is reduced in case of convergence failure or other problems (default is 4). If REDLT<0.0, REDLT*(-1.0) will be used as increasing rate for time-step size.

SCALE Scale factor to change the size of the mesh (default $=1.0$ ).

Record PARAM.2.1.1 (optional, ELST = RICKA only)

Format (I10)

NELIST

NELIST Specifies the total number of elements $(>1)$ for which time versus primary variables is printed at each time step into files: FOFT_P.xxx. The file extension $\mathrm{xxx}$ is the identification number of the processor at which the output was generated.

Record ROCKS.2.1.2, 2.1.3, etc (optional, ELST = RICKA only) Number of records $=$ NELIST

Format (A5) for MOP $(14)=0$ or Format $(\mathrm{A} 8)$ for $\mathrm{MOP}(14)=1$. EPLIST(I)

EPLIST(I), $\quad \mathrm{I}=1,2, \ldots$, NELIST, element's names for which time versus primary variables needs to be printed at each time step into files: FOFT_P.xxx. 
Record PARAM.2.2.1, 2.2.2, etc.

Format (8E10.4)

(DLT(I), I = 1, 100)

DLT(I) Length (in seconds) of time step I.

This set of records is optional for DELTEN = - NDLT, a negative integer. Up to 13 records can be read, each containing 8 time step data. If the number of simulated time steps exceeds the number of DLT(I), the simulation will continue with time steps equal to the last non-zero DLT(I) encountered. When automatic time step control is chosen $(\operatorname{MOP}(16)>0)$, time steps following the last DLT(I) input by the user will increase according to the convergence rate of the Newton-Raphson iteration. Automatic time step reduction will occur if the maximum number of NewtonRaphson iterations is exceeded (parameter NOITE, record PARAM.1)

$\underline{\text { Record PARAM.3 }}$

Format (6E10.4)

RE1, RE2, U, WUP, WNR, DFAC

RE1 Convergence criterion for relative error (default $=10^{-5}$ ).

RE2 Convergence criterion for absolute error, see (default=1).

U Not be used

WUP Upstream weighting factor for mobilities and enthalpies at interfaces (default $=1.0$ is recommended). $0 \leq \mathrm{WUP} \leq 1$.

WNR Weighting factor for increments in Newton/Raphson - iteration (default $=1.0$ is recommended). $0<\mathrm{WNR} \leq 1$.

DFAC Increment factor for numerically computing derivatives (default value is DFAC $=10^{-k / 2}$, where $\mathrm{k}$, evaluated internally, is the number of significant digits of the floating point processor used; for 64-bit arithmetic, DFAC $\approx 10^{-8}$ ). 
Record PARAM.4 Introduces a set of primary variables which are used as default initial conditions for all gridblocks that are not assigned by means of data blocks INDOM or INCON. Option START is necessary to use default INCON.

Format (4E20.14)

$\operatorname{DEP}(\mathrm{I}), \mathrm{I}=1, \mathrm{NK}+1$

The number of primary variables, $\mathrm{NK}+1$, is normally assigned internally in the EOS module, and is usually equal to the number NEQ of equations solved per gridblock. See data block MULTI for special assignments of NK. Different sets of primary variables are in use for different EOS modules.

INDOM introduces domain-specific initial conditions. These will supersede default initial conditions specified in PARAM.4, and can be overwritten by element-specific initial conditions in data block INCON. Option START is needed to use INDOM conditions.

Record INDOM. 1

Format(A5)

MAT

MAT Name of a reservoir domain, as specified in data block ROCKS.

Record INDOM.2

Format(4E20.13)

$\mathrm{X} 1, \mathrm{X} 2, \mathrm{X} 3, \ldots \ldots$

A set of primary variables assigned to all gridblocks in the domain specified in record INDOM. 1. Different sets of primary variables are used for different EOS modules.

$\underline{\text { Record INDOM.3 }}$ 
A blank record closes the INDOM data block. Repeat records INDOM. 1 and INDOM.2 for as many domains as desired. The ordering is arbitrary and need not be the same as in block ROCKS.

INCON introduces element-specific initial conditions.

Record INCON.1

For $\operatorname{MOP}(14)=0$, 5-character element

Format (A3, I2, 2I5,E15.9)

EL, NE, NSEQ, NADD, PORX

For $\operatorname{MOP}(14)=1,8$ character element

Format (A6, I2)

EL, NE

EL, NE Code name of element.

NSEQ Number of additional elements with the same initial conditions (used only for 5-character element name).

NADD Increment between the code numbers of two successive elements with identical initial conditions (used only for 5-character element name).

PORX Porosity; if zero or blank, porosity will be taken as specified in block ROCKS if option START is used.

Record INCON.2 specifies primary variables.

Format (4E20.14)

X1, X2, X3, X4

A set of primary variables for the element specified in record INCON.1. INCON specifications will supersede default conditions specified in PARAM.4, and domain-specific conditions that may have been specified in data block INDOM. Different sets of primary variables are used for different EOS modules.

Record INCON.3 A blank record closes the INCON data block. Alternatively, initial condition information may terminate on a record with "+++" typed in the first three columns, followed by 
time stepping information. This feature is used for a continuation run from a previous TOUGH2-MP simulation.

NOVER (optional)

One record with NOVER typed in columns 1-5 will suppress printing of a summary of versions and dates of the program units used in a TOUGH2-MP run.

SELEC (optional) introduces a number of integer and floating point parameters that are used for different purposes in different TOUGH2 modules.

$\underline{\text { Record SELEC. } 1}$

Format(16I5)

$\mathrm{IE}(\mathrm{I}), \mathrm{I}=1,16$

IE(1) number of records with floating point numbers that will be read (default is $\operatorname{IE}(1)=1$; maximum values is 64).

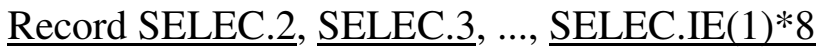

Format $(8 \mathrm{E} 10.4)$

$\mathrm{FE}(\mathrm{I}), \mathrm{I}=1, \mathrm{IE}(1) * 8$

Provide as many records with floating point numbers as specified in $\mathrm{IE}(1)$, up to a maximum of 64 records.

RPCAP introduces information on relative permeability and capillary pressure functions, which will be applied for all flow domains for which no data were specified in records ROCKS.1.2 and ROCKS.1.3. A catalog of relative permeability and capillary pressure functions is presented in Appendix B and Appendix C, respectively.

\section{$\underline{\text { Record RPCAP.1 }}$}

Format (I5,5X,7E10.4)

$\mathrm{IRP},(\mathrm{RP}(\mathrm{I}), \mathrm{I}=1,7)$ 
IRP Integer parameter to choose type of relative permeability function (see Appendix B).

$\mathrm{RP}(\mathrm{I}), \mathrm{I}=1, \ldots, 7$ parameters for relative permeability function (Appendix B).

\section{Record RPCAP.2}

Format (I5,5X,7E10.4)

$\mathrm{ICP},(\mathrm{CP}(\mathrm{I}), \mathrm{I}=1,7)$

ICP Integer parameter to choose type of capillary pressure function (see Appendix C).

$\mathrm{CP}(\mathrm{I}) \quad \mathrm{I}=1, \ldots, 7$ parameters for capillary pressure function (Appendix $\mathrm{C}$ ).

TIMES permits the user to obtain printout at specified times (optional). This printout will occur in addition to printout specified in record PARAM.1.

$\underline{\text { Record TIMES.1 }}$

Format $(2 \mathrm{I} 5,2 \mathrm{E} 10.4)$

ITI, ITE, DELAF, TINTER

ITI Number of times provided on records TIMES.2, TIMES.3, etc., (see below; restriction: $\mathrm{ITI} \leq 100$ ).

ITE Total number of times desired (ITI $\leq$ ITE $\leq 100$; default is ITE $=$ I TI).

DELAF Maximum time step size after any of the prescribed times have been reached (default is infinite).

TINTER Time increment for times with index ITI, ITI+1, .., ITE.

Record TIMES.2, TIMES.3, etc.

Format (8E10.4)

(TIS(I), I = 1, ITI)

TIS(I) List of times (in ascending order) at which printout is desired. 
ELEME introduces element (gridblock) information. See Section 4.4 for additional explanations.

$\underline{\text { Record ELEME.1 }}$

For $\operatorname{MOP}(14)=0$, 5-character element

Format (A3, I2, 2I5, A3, A2, 6E10.4)

EL, NE, NSEQ, NADD, MA1, MA2, VOLX, AHTX, PMX, X, Y, $\mathrm{Z}$

For $\operatorname{MOP}(14)=1,8$-character element

Format (A6, I2, 7X, A3, A2, 6E10.4)

EL, NE, MA1, MA2, VOLX, AHTX, PMX, X, Y, Z

EL, NE Five-character (or eight-character with $\operatorname{MOP}(14)=1$ ) code name of an element. The first three or six characters are arbitrary; the last two characters must be numbers.

NSEQ Number of additional elements having the same volume and belonging to the same reservoir domain (Only for $\operatorname{MOP}(14)=0)$.

NADD Increment between the code numbers of two successive elements. $($ Only for $\operatorname{MOP}(14)=0)$

MA1, MA2 A five-character material identifier corresponding to one of the reservoir domains as specified in block ROCKS. If the first three characters are blanks and the last two characters are numbers then they indicate the sequence number of the domain as entered in ROCKS. If both MA1 and MA2 are left blank the element is by default assigned to the first domain in block ROCKS.

VOLX Element volume $\left(\mathrm{m}^{3}\right)$.

AHTX Interface area $\left(\mathrm{m}^{2}\right)$ for heat exchange with semi-infinite confining beds.

PMX permeability modifier (optional, active only when a domain 'SEED' has been specified in the ROCKS block; see TOUGH2 V2 User's Guide). It will be used as multiplicative factor for the permeability parameters from block ROCKS. Simultaneously, 
strength of capillary pressure will be scaled as 1/SQRT(PMX). PMX $=0$ will result in an impermeable block.

Random permeability modifiers can be generated internally, see detailed comments in the TOUGH2-MP output file. The PMX may be used to specify spatially correlated heterogeneous fields, but users need their own preprocessing programs for this, as TOUGH2 provides no internal capabilities for generating such fields.

$\mathrm{X}, \mathrm{Y}, \mathrm{Z} \quad$ Cartesian coordinates of gridblock centers. These may be included in the ELEME data to make subsequent plotting of results more convenient. The coordinate data are not used internally by TOUGH2-MP, except with EOS9 for initialization of a gravitycapillary equilibrium.

Repeat record ELEME.1 for the number of elements desired.

Record ELEME.2 A blank record closes the ELEME data block.

CONNE introduces information for the connections (interfaces) between elements. See Section 4.4 for additional explanations.

Record CONNE. 1

For $\operatorname{MOP}(14)=0$, 5-character element

Format (A3, I2, A3, I2, 4I5, 5E10.4)

EL1, NE1, EL2, NE2, NSEQ, NAD1, NAD2, ISOT, D1, D2, AREAX, BETAX, SIGX/IFM_CON

For $\operatorname{MOP}(14)=1,8$-character element

Format (A6, I2, A6, I2, 9X, I5, 5E10.4)

EL1, NE1, EL2, NE2, ISOT, D1, D2, AREAX, BETAX, SIGX/IFM_CON

EL1, NE1 Code name of the first element.

EL2, NE2 Code name of the second element.

NSEQ Number of additional connections in the sequence (for MOP(14)=0 only). 
NAD1 Increment of the code number of the first element between two successive connections (for $\operatorname{MOP}(14)=0$ only).

NAD2 Increment of the code number of the second element between two successive connections (for $\mathrm{MOP}(14)=0$ only).

ISOT Set equal to 1,2 , or 3; specifies absolute permeability to be PER(ISOT) for the materials in elements (EL1, NE1) and (EL2, NE2), where PER is read in block ROCKS. This allows assignment of different permeabilities, e.g., in the horizontal and vertical direction.

Note that in this version, several schemes of fracture-matrix (F-M) interface area reduction for F-M local connection and mobility weighting are implemented using ISOT, which is set to a negative integer as follows:

$=-1 \quad$ F-M interconnection area used for calculating flow of a fluid is multiplied by the upstream saturation of the fluid,

$=-2$ mobility of the lower absolute permeability block is used for flow calculation along this connection.

$=-3 \quad$ F-M interconnection area for calculating flow of a fluid is multiplied by a constant factor ( $=\mathrm{RP}(7)$ from fracture rock material) and by the upstream relative permeability to the fluid. This scheme is called weeps type model.

$=-4 \quad$ F-M interconnection area for calculating flow of a fluid is multiplied by the upstream relative permeability to the fluid, $=-9 \quad \mathrm{~F}-\mathrm{M}$ interconnection area for calculating flow of a fluid is multiplied by a constant factor $(=\mathrm{RP}(6)$ from fracture rock material),

$=-10$ F-M interconnection area for calculating flow of the liquid is modified by the active fracture model (Liu et al., 1998). 
D1 Distance (m) from first element to common interface.

D2 Distance (m) from second element to common interface.

AREAX Interface area $\left(\mathrm{m}^{2}\right)$.

BETAX Cosine of the angle between the gravitational acceleration vector and the line between the two elements. GF $*$ BETAX $>0(<0)$ corresponds to first element being above (below) the second element.

IFM_CON Connection identifier,

(For Ver 1.4) = 0: for connection between single-continuum, matrix, and/or ECM elements,

$=1.0$ : for connection between fracture elements in a dualcontinuum model (dual-permeability, double-porosity, MINC, etc.), = 2.0: for local connection between fracture-matrix elements in a dual-continuum grid (dual-permeability, double-porosity, MINC, etc.), and

= 3.0: for global connection between fracture-matrix, fractureECM, or fracture-single-continuum elements in a hybrid, dualcontinuum grid (a combined, single-continuum, ECM, dualpermeability, double-porosity, and MINC, etc.).

SIGX "radiant emittance" factor for radiative heat transfer, which for a

(For Ver 2.0) perfectly "black" body is equal to 1 . The rate of radiative heat transfer between the two grid blocks is $G_{r a d}=S I G X * \sigma_{0} * A R E A X *\left(T_{2}^{4}-T_{1}^{4}\right)$

where $\sigma_{0}=5.6687 \mathrm{e}-8 \mathrm{~J} / \mathrm{m}^{2} \mathrm{~K}^{4} \mathrm{~s}$ is the Stefan-Boltzmann constant, and $\mathrm{T} 1$ and $\mathrm{T} 2$ are the absolute temperatures of the two grid blocks. SIGX may be entered as a negative number, in which case the absolute value will be used, and heat conduction at the connection will be suppressed. SIGX $=0$ will result in no radiative heat transfer. 
Repeat record CONNE.1 for the number of connections desired.

Record CONNE.2 A blank record closes the CONNE data block. Alternatively, connection information may terminate on a record with '+++' typed in the first three columns, followed by element cross-referencing information. This is the termination used when generating a MESH file with TOUGH2.

GENER introduces sinks and/or sources.

Record GENER. 1

For $\operatorname{MOP}(14)=0,5$-character element

Format (A3, I2, A3, I2, 4I5, 5X, A4, A1, 3E10.4)

EL, NE, SL, NS, NSEQ, NADD, NADS, LTAB, TYPE, ITAB, GX, EX, HX

For $\operatorname{MOP}(14)=1,8$-character element

Format (A6, I2, A3, I2, 12X, I5, 5X, A4, A1, 3E10.4)

EL, NE, SL, NS, LTAB, TYPE, ITAB, GX, EX, HX

EL, NE Code name of the element containing the sink/source.

SL, NS Code name of the sink/source. The first three characters are arbitrary, the last two characters must be numbers.

NSEQ Number of additional sinks/sources with the same injection/production rate (not implemented in TOUGH2-MP).

NADD Increment between the code numbers of two successive elements with identical sink/source (not implemented in TOUGH2-MP).

NADS Increment between the code numbers of two successive sinks/sources (not implemented in TOUGH2-MP).

LTAB Number of points in table of generation rate versus time. Set 0 or 1 for constant generation rate. For wells on deliverability, LTAB denotes the number of open layers, to be specified only for the bottommost layer. 
TYPE Specifies different options for fluid or heat production and injection. For example, different fluid components may be injected, the nature of which depends on the EOS module being used. Different options for considering wellbore flow effects may also be specified.

HEAT introduces a heat sink/source.

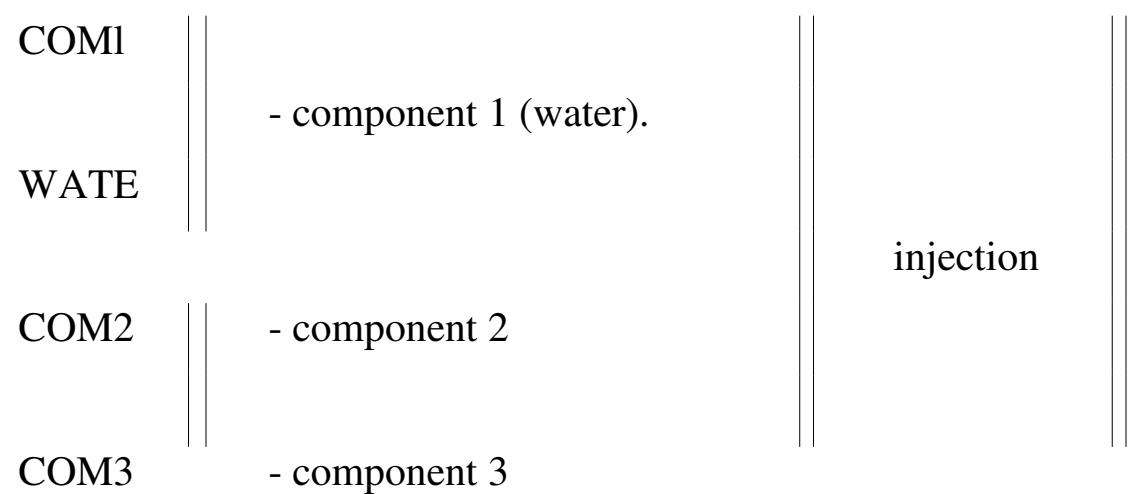

MASS-mass production rate specified.

DELV-well on deliverability, i.e., production occurs against specified bottomhole pressure. If well is completed in more than one layer, bottommost layer must be specified first, with number of layers given in LTAB. Subsequent layers must be given sequentially for a total number of LTAB layers.

ITAB Unless left blank, table of specific enthalpies will be read (LTAB > 1 only).

GX Constant generation rate; positive for injection, negative for production; $\mathrm{GX}$ is mass rate $(\mathrm{kg} / \mathrm{sec})$ for generation types COMl, COM2. COM3, etc., and MASS; it is energy rate $(\mathrm{J} / \mathrm{s})$ for a HEAT sink/source. For wells on deliverability, GX is productivity index PI $\left(m^{3}\right)$.

EX Fixed specific enthalpy $(\mathrm{J} / \mathrm{kg})$ of the fluid for mass injection $(\mathrm{GX}>0)$. For wells on deliverability against fixed bottomhole 
pressure, $\mathrm{EX}$ is bottomhole pressure $\mathrm{P}_{\mathrm{wb}}(\mathrm{Pa})$, at the center of the topmost producing layer in which the well is open.

HX Tickness of layer $(\mathrm{m}$; wells on deliverability with specified bottomhole pressure only).

Record GENER.1.1 (optional, LTAB > 1 only)

Format (4E14.7)

$\mathrm{Fl}(\mathrm{L}), \mathrm{L}=1, \mathrm{LTAB}$

F1 Generation times

Record GENER.1.2 (optional, LTAB $>1$ only)

Format (4E14.7)

F2(L), L=1, LTAB

F2 Generation rates.

Record GENER.1.3 (optional, LTAB > 1 and ITAB non-blank only)

Format (4E14.7)

F3(L), L=1, LTAB

F3 Specific enthalpy of produced or injected fluid.

Repeat records GENER.1, 1.1, 1.2, and 1.3 for the number of sinks/sources desired.

Record GENER.2 A blank record closes the GENER data block.

Alternatively, generation information may terminate on a record with ' +++ ' typed in the first three columns, followed by element cross-referencing information.

DIFFUSION (optional; needed only for $\mathrm{NB} \geq 8$, for Ver 2.0 only) introduces diffusion coefficients.

Record DIFFU.1

Format(8E10.4)

FDDIAG(I,1), I=1,NPH

diffusion coefficients for mass component \# 1 in all phases (I=1: gas; I=2: aqueous; etc.)

Record DIFFU.2 
Format(8E10.4)

FDDIAG(I,2), I=1,NPH

diffusion coefficients for mass component \# 2 in all phases ( $\mathrm{I}=1$ :

gas; I=2: aqueous; etc.)

Provide a total of NK records with diffusion coefficients for all NK mass components. See Pruess et al. (1999) for additional parameter specifications for diffusion.

FOFT (optional) introduces a list of elements (grid blocks) for which time dependent data are to be written out for plotting to a file called FOFT_P.xxx during the simulation. The file extension $\mathrm{xxx}$ is the identification number of the processor at which the output was generated.

Record FOFT.1

Format(A5) (for MOP(14)=0)

Format(A8) (for MOP(14)=1)

EOFT(I)

EOFT is an element name. Repeat for up to 100 elements, one per record.

Record FOFT.2 A blank record closes the FOFT data block.

COFT (optional) introduces a list of connections for which timedependent data are to be written out for plotting to file FOFT_P.xxx during the simulation.

Record COFT.1

Format (A10) (for MOP $(14)=0)$

Format(A16) (for MOP(14)=1)

ECOFT(I) 
ECOFT is a connection name, i.e., an ordered pair of two element names. Repeat for up to 100 connections, one per record.

Record COFT.2 A blank record closes the COFT data block.

GOFT (optional) introduces a list of sinks/sources for which timedependent data are to be written out for plotting to file FOFT_P.xxx during the simulation.

Record GOFT.1

Format(A5) (for $\operatorname{MOP}(14)=0$ )

Format(A8) (for $\operatorname{MOP}(14)=1$ )

EGOFT(I)

EGOFT is the name of an element in which a sink/source is defined. Repeat for up to 100 sinks/sources, one per record. When no sinks or sources are specified here, by default tabulation will be made for all.

Record GOFT.2 A blank record closes the GOFT data block.

TIMBC (optional) introduces a table (external data file, named as "timvsp.dat", must be located at the simulation working directory) for time-dependent pressure boundary conditions.

File "timevsp.dat" format:

FORMAT(2I5)

NPOINT, NTPTAB (number of time points and gridblocks at which pressure boundary conditions will be specified)

FORMAT(4E14.7) 
TIMBCV(I), I=1, NPOINT (times for each time point)

FORMAT(A5) for $\mathrm{MOP}(14)=0$, and FORMAT(A8) for $\operatorname{MOP}(14)=1$

BCELEM(I), I=1, NTPTAB (name list of the NTPRAB gridblocks)

FORMAT(4E14.7)

PGBCEL(I,J), I=1, NPOINT; J=1,NTPTAB (boundary pressure provided at the NPONIT time points for all the NTPTAB gridblocks

RTSOL (optional) introduces additional time stepping, iteration and solver parameters. This keyword inherits from VER 1.4. Most parameters under this keyword have not been used in TOUGH2-MP.

Record RTSOL.1

Format $(2 \mathrm{E} 10.3,6 \mathrm{I} 5)$

PREC, RTOL, INFO, IPLVL, NITMX, NORT, KACCEL, IREDB

All these parameters have not been used in this version.

Record RTSOL.2

Format (7F10.3,I5)

DTMIN, DTMAX, DSTNOM, DXTMAX,TMULFC, RELXSN, RELXXN, ICOLEY

DTMIN minimum time step size in seconds.

DSTNOM maximum allowable saturation change per time step (default=0.2).

TMULFC Time step size increasing rate when Newton iteration converges in less then MOP(16) iterations, see also REDLT.

ICOLEY flag for evaluating an underrelaxation factor for updating primary variables over Newton iteration;

$=0$ : if no underrelaxation scheme is used, $=1$ : if Cooley underrelaxation scheme is used, and 
$=2$ : if an underrelaxation scheme is determined using DSTNOM, normalized maximum changes in saturation.

Other parameters have not been used in this version.

ENDCY closes the TOUGH2 input file and initiates the simulation.

\section{Note on closure of blocks CONNE, GENER, and INCON}

The conventional way to indicate the end of any of the above data blocks is by means of a blank record. There is an alternative available if the user constructs an input file from files MESH, GENER, or SAVE, which have been generated by a previous TOUGH2 or TOUGH2-MP run. These files are written exactly according to the specifications of data blocks ELEME and CONNE (file MESH), GENER (file GENER), and INCON (file SAVE), except that the CONNE, GENER, and INCON data terminate on a record with “+++" in Columns 1-3, followed by some cross-referencing (indexing) and restart information. TOUGH2-MP will accept this type of input, and in this case there is no blank record at the end of an indicated data block. The cross-referencing information will not be read by the parallel code, because this information may be not correct when the model has a total of more than 100,000 gridblocks. The parallel code uses a very efficient index searching algorithm that computes the connection and gridblock indices at the beginning of every simulation run.

\subsection{Input Formats for MESHMAKER}

The MESHMaker module performs internal mesh generation and processing. This module has not been parallelized and is run on the master processor only. In general, the input and output of MESHMaker for TOUGH2-MP are identical to V2.0, except that the parallel version allows generating multi-million gridblocks for Cartesian X-Y-Z mesh.

The input for MESHMaker has a modular structure and a variable number of records; it begins with keyword MESHM and ends with a blank record.

There are three submodules available in MESHMaker: keywords RZ2D or RZ2DL invoke generation of a one or two-dimensional radially symmetric R-Z mesh; XYZ 
initiates generation of a one, two, or three-dimensional Cartesian X-Y-Z mesh; and MINC calls a modified version of the GMINC program (Pruess, 1983) to subpartition a primary porous medium mesh into a secondary mesh for fractured media, using the method of "multiple interacting continua" (Pruess and Narasimhan, 1985). The meshes generated under keyword RZ2D or XYZ are internally written to file MESH. The MINC processing operates on the data in file MESH, so that invoking the RZ2D or XYZ options, or assignment of ELEME and CONNE blocks in the INPUT file, must precede the MESHMaker/MINC data. We shall now separately describe the preparation of input data for the three MESHMaker submodules.

\subsubsection{Generation of Radially Symmetric Grids}

Keyword RZ2D (or RZ2DL) invokes generation of a radially symmetric mesh. Values for the radii to which the gridblocks extend can be provided by the user or can be generated internally (see below). Nodal points will be placed half-way between neighboring radial interfaces. When RZ2D is specified, the mesh will be generated by columns; i.e., in the ELEME block, we will first have the gridblocks at smallest radius for all layers, then the next largest radius for all layers, and so on. With keyword RZ2DL, the mesh will be generated by layers; i.e., in the ELEME block, we will first have all gridblocks for the first (top) layer from smallest to largest radius, then all gridblocks for the second layer, and so on. Apart from the different ordering of elements, the two meshes for RZ2D and RZ2DL are identical. Assignment of inactive elements would be made by using a text editor on the RZ2D-generated MESH file, and moving groups of elements towards the end of the ELEME block, past a dummy element with zero volume. RZ2D makes it easy to declare a vertical column inactive, facilitating assignment of boundary conditions in the vertical, such as a gravitationally equilibrated pressure gradient. RZ2DL, on the other hand, facilitates implementation of areal (top and bottom layer) boundary conditions. 
RADII is the first keyword following RZ2D; it introduces data for defining a set of interfaces (gridblock boundaries) in the radial direction.

$\underline{\text { Record RADII.1 }}$

Format(I5)

NRAD

NRAD Number of radius data that will be read. At least one radius must be provided, indicating the inner boundary of the mesh.

Record RADII.2, RADII.3, etc.

Format(8E10.4)

$\mathrm{RC}(\mathrm{I}), \mathrm{I}=1$, NRAD

$\mathrm{RC}(\mathrm{I}) \quad$ A set of radii in ascending order.

EQUIDistant introduces data on a set of equal radial increments.

Record EQUID. 1

Format(I5, 5X, E10.4)

NEQU, DR

NEQU Number of desired radial increments.

DR Magnitude of radial increment.

Note: At least one radius must have been defined via block RADII before EQUID can be invoked.

LOGARithmic introduces data on radial increments that increase from one to the next by the same factor $\left(\Delta R_{n+l}=f \cdot \Delta R_{n}\right)$.

$\underline{\text { Record LOGAR. } 1}$

Format(A5, 5X, 2E10.4)

NLOG, RLOG, DR

NLOG number of additional interface radii desired.

RLOG Desired radius of the last (largest) of these radii. 
$\mathrm{DR}$ reference radial increment: the first $\Delta \mathrm{R}$ generated will be equal to $\mathrm{f} \cdot \mathrm{DR}$, with $\mathrm{f}$ internally determined such that the last increment will bring total radius to RLOG. $\mathrm{f}<1$ for decreasing radial increments is permissible. If DR is set equal to zero, or left blank, the last increment DR generated before keyword LOGAR will be used as default.

Additional blocks RADII, EQUID, and LOGAR can be specified in arbitrary order.

Note: At least one radius must have been defined before LOGAR can be invoked. If $\mathrm{DR}=0$, at least two radii must have been defined.

LAYER introduces information on horizontal layers, and signals closure of RZ2D input data.

$\underline{\text { Record LAYER. } 1}$

Format(I5)

NLAY

NLAY Number of horizontal grid layers.

Record LAYER.2

Format(8E10.4)

$\mathrm{H}(\mathrm{I}), \mathrm{I}=1$, NLAY

H(I) A set of layer thicknesses, from top layer downward. By default, zero or blank entries for layer thickness will result in assignment of the last preceding nonzero entry. Assignment of a zero layer thickness, as needed for inactive layers, can be accomplished by specifying a negative value.

The LAYER data close the RZ2D data block. Note that one blank record must follow to indicate termination of the MESHM data block. Alternatively, keyword MINC can appear to invoke MINCprocessing for fractured media (see below). 


\subsubsection{Generation of Rectilinear Grids}

XYZ invokes generation of a Cartesian (rectilinear) mesh.

$\underline{\text { Record XYZ.1 }}$

Format(E10.4)

DEG

DEG Angle (in degrees) between the Y-axis and the horizontal. If gravitational acceleration (GF in record PARAM.2) is specified positive, $-90^{\circ}<\mathrm{DEG}<90^{\circ}$ corresponds to grid layers going from top down. Grids can be specified from bottom layer up by setting GF or BETA negative. Default $(\mathrm{DEG}=0)$ corresponds to horizontal Y-and vertical Z-axis. X-axis is always horizontal.

$\underline{\text { Record XYZ.2 }}$

Format(A2, 3X, I5, E10.4)

NTYPE, NO, DEL

NTYPE Set equal to NX, NY or NZ for specifying grid increments in X, Y, or $\mathrm{Z}$ direction.

NO Number of grid increments desired.

DEL Constant grid increment for NO gridblocks, if set to a non zero value.

$\underline{\text { Record XYZ.3 }}$ (optional, DEL $=0$. or blank only)

Format(8E10.4)

$\operatorname{DEL}(\mathrm{I}), \mathrm{I}=1$, NO

DEL(I) A set of grid increments in the direction specified by NTYPE in record XYZ.2. Additional records with formats as XYZ.2 and XYZ.3 can be provided, with X, Y, and Z-data in arbitrary order.

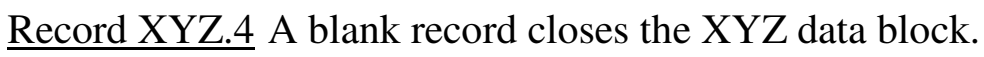

Note that the end of block MESHMaker is also marked by a blank record. Thus, when MESHMaker/XYZ is used, there will be two blank records at the end of the corresponding input data block. 


\subsubsection{MINC Processing for Fractured Media}

MINC invokes postprocessing of a primary porous medium mesh from file MESH. The input formats in data block MINC are identical to those of the GMINC program (Pruess, 1983), with two enhancements: there is an additional facility for specifying global matrix-matrix connections ("dual permeability"); further, only active elements will be subjected to MINC-processing, the remainder of the MESH remaining unaltered as porous medium gridblocks.

PART is the first keyword following MINC; it will be followed on the same line by parameters TYPE and DUAL with information on the nature of fracture distributions and matrix-matrix connections.

Format(2A5, 5X, A5)

PART, TYPE, DUAL

PART Identifier of data block with partitioning parameters for secondary mesh.

TYPE A five-character word for selecting one of the six different proximity functions provided in MINC (Pruess, 1983).

ONE-D: $\quad$ a set of plane parallel infinite fractures with matrix block thickness between neighboring fractures equal to PAR(l).

TWO-D: two sets of plane parallel infinite fractures, with arbitrary angle between them. Matrix block thickness is PAR(1) for the first set, and PAR(2) for the second set. If $\operatorname{PAR}(2)$ is not specified explicitly, it will be set equal to PAR(1). 
THRED: three sets of plane parallel infinite fractures at right angles, with matrix block dimensions of $\operatorname{PAR}(1)$, $\operatorname{PAR}(2)$, and $\operatorname{PAR}(3)$, respectively. If $\operatorname{PAR}(2)$ and/or PAR(3) are not explicitly specified, they will be set equal to $\operatorname{PAR}(1)$ and/or $\operatorname{PAR}(2)$, respectively.

Note: a user wishing to employ a different proximity function than provided in MINC needs to replace the function subprogram $\operatorname{PROX}(\mathrm{x})$ in file meshm.f with a routine of the form:

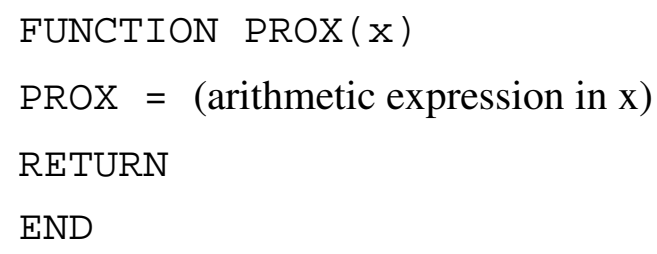

It is necessary that $\operatorname{PROX}(\mathrm{x})$ is defined even when $\mathrm{x}$ exceeds the maximum possible distance from the fractures, and that PROX $=1$ in this case. Also, when the user supplies his/her own proximity function subprogram, the parameter TYPE has to be chosen equal to ONE-D, TWO-D, or THRED, depending on the dimensionality of the proximity function. This will assure proper definition of the innermost nodal distance (Pruess, 1983).

DUAL A five-character word for selecting the treatment of global matrix flow.

blank: (default) Global flow occurs only through the fracture continuum, while rock matrix and fractures interact locally by means of interporosity flow (double-porosity model).

MMVER: $\quad$ global matrix-matrix flow is permitted only in the vertical; otherwise like the double-porosity model; for internal consistency this choice should only be made for flow systems with one or two predominantly vertical fracture sets. 
MMALL: Global matrix-matrix flow in all directions; for internal consistency only two continua, representing matrix and fractures, should be specified (“dual-permeability”).

$\underline{\text { Record PART.1 }}$

Format (2I3, A4, 7E10.4)

J, NVOL, WHERE, (PAR(I), I = 1, 7)

J Total number of multiple interacting continua $(\mathrm{J}<36)$.

NVOL Total number of explicitly provided volume fractions $(\mathrm{NVOL}<\mathrm{J})$.

If $\mathrm{NVOL}<\mathrm{J}$, the volume fractions with indices NVOL $+1, \ldots, \mathrm{J}$ will be internally generated; all being equal and chosen such as to yield proper normalization to 1 .

WHERE Specifies whether the sequentially specified volume fractions begin with the fractures (WHERE = 'OUT ') or in the interior of the matrix blocks (WHERE = 'IN ').

$\operatorname{PAR}(\mathrm{I}), \mathrm{I}=1,7 \quad$ Holds parameters for fracture spacing (see above).

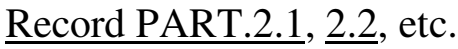

Format (8E10.4)

(VOL(I), I = 1, NVOL)

VOL(I) Volume fraction (between 0 and 1) of continuum with index I (for WHERE = 'OUT ') or index J+ 1 - I (for WHERE = 'IN '). NVOL volume fractions will be read. For WHERE = 'OUT ', I = 1 is the fracture continuum, $I=2$ is the matrix continuum closest to the fractures, $I=3$ is the matrix continuum adjacent to $I=2$, etc. The sum of all volume fractions must not exceed 1.

\subsection{Special Input Requirements for TOUGH2-MP}

In some cases, TOUGH2-MP needs to be run in batch mode. To run a job in batch mode, the user submits a job to a computer and the computer schedules the job in a queue. When the requested number of processors is available, the job will be run. In batch running mode, all data are provided in input files, since run-time communication is not 
feasible. For both batch and interactive mode, the input files for the parallel run include:

\section{INFILE}

This file is in the same data format as a TOUGH2 input file, as discussed in Section 4.2. In this input file, data are organized in blocks that are defined by five-character keywords typed in Columns 1-5. The first record must be a problem title of up to 80 characters. The last record usually is ENDCY. Data records beyond ENDCY will be ignored. The most important data blocks include ROCKS, MULTI, PARAM, ELEME, CONNE, INCON, and GENER. All input data in INFILE are in fixed format and standard metric (SI) units. Detailed information about this file format can be found in Section 4.2

The blocks of ELEME, CONNE, GENER and INCON can be extremely large. It is good practice to provide these blocks through separate data files. An alternative input for ELEME and CONNE blocks is through the MESH file or through two binary files: MESHA and MESHB. The two binary files are intermediate files which are created by TOUGH2-MP during its first run for a model. If MESHA and MESHB exist in the working folder, the code will ignore MESH file and read information directly from these two files. If the mesh is changed, MESHA and MESHB must be deleted from the working folder to make the changes take effect. The two files have completely different data formats from the ELEME and CONNE blocks. The detailed format information is given in the following.

\section{MESHA, MESHB}

The purpose of replacing file MESH (or blocks ELEME and CONNE in an input file) with MESHA and MESHB is to reduce the memory requirement for the master processor and to enhance I/O efficiency. Both MESHA and MESHB are binary files. These two files contain all information provided by file MESH. There are two groups of large data blocks within a TOUGH2 mesh file: one with dimensions equal to the number of gridblocks, the other with dimensions equal to the number of connections (interfaces). To

read and use computer memory efficiently, the input data are organized in sequential and binary format. Large data blocks are read one by one through a temporary full-size array 
and then distributed to processors one by one. This method avoids storing all input data in one single processor and enhances the I/O efficiency and total storage capacity.

The file MESHA is written (to file unit 20 that was opened as an unformatted file) in the following sequence:

\section{[Keni: somewhere NCON must be written; please correct this.]}

write(20) NEL

write(20) $(E V O L(i I), i I=1, N E L)$

write(20) $(A H T(i I), i I=1, N E L)$

write(20) $(P M X(i I), i I=1, N E L)$

write(20) $(\operatorname{gcoord}(i I, 1), i I=1, N E L)$

write(20) (gcoord(iI,2),iI=1,NEL)

write $(20)(\operatorname{gcoord}(i I, 3), i I=1, N E L)$

write(20) (DEL1(iI), iI=1,NCON)

write(20) (DEL2(iI), $i I=1, N C O N)$

write(20) (AREA(iI), $i I=1, N C O N)$

write(20) (BETA(iI), $i I=1, N C O N)$

write(20) (SIG(iI), $i I=1, N C O N)$

write (20) $(I S O X(i I), i I=1, N C O N)$

write $(20)(\operatorname{ELEMI}(i \mathrm{I}), i \mathrm{I}=1, \mathrm{NCON})$

write(20)(ELEM2(iI), iI=1,NCON)

where

NEL Total gridblock number, in 8-byte integer.

NCON Total connection number, in 8-byte integer.

EVOL Element volume $\left(\mathrm{m}^{3}\right)$, in 8-byte real

AHT Interface area $\left(\mathrm{m}^{2}\right)$ for heat exchange with semi-infinite confining beds, in 8-byte real.

PMX Permeability modifier, in 8-byte real gcoord $(*, 1-3)$ Cartesian coordinates $(\mathrm{X}, \mathrm{Y}, \mathrm{X})$ of gridblock center, in 8-byte real. 
DEL1, DEL2 Distance (m) from first and second element, respectively, to their common interface, in 8-byte real.

AREA Interface area $\left(\mathrm{m}^{2}\right)$, in 8-byte real.

BETA Cosine of the angle between the gravitational acceleration vector and the line between two elements, in 8-byte real.

SIG "Radiant emittance" factor for radiative heat transfer (Ver2.0), or for defining the connection property, the connection can be between fractures, matrices, or fracture and matrix (Ver 1.4), in 8-byte real.

ISOX Specify absolute permeability for the connection, in 4-byte integer.

ELEM1 Code name for the first element of a connection, in 8 characters.

ELEM2 Code name for the second element of a conection, in 8 character.

The file MESHB is written (to file unit 30, unformatted) in the following sequence:

write(30) NCON,NEL

write(30) $(\operatorname{ELEM}(i I), i I=1, N E L)$

write(30) (MA12(iI),iI=1,NEL)

write(30) $(N E X 1(i I), i I=1, N C O N)$

write(30) $(N E X 2(i I), i I=1, N C O N)$

where

ELEM Code name of the element, in 8 characters.

MA12 Material identifier of the element, in 5 characters.

NEX1, NEX2 First and second element number of the connection, in 4-byte integer.

For more detailed explanation of these parameters, the reader may refer to the TOUGH2 User's Guide, Version 2.0 (Pruess et al., 1999).

After a first run of each simulation, the material name array MA12 will be replaced by the material index (array MATX, in 8-byte integer). In addition, NEL will be replaced by 
-NEL to inform the program of the replacement. Through this replacement, the material index searching is avoided for future runs.

MESHA and MESHB can also be created directly from MESH file through a preprocessing program. For extremely large problems, generation of MESHA and MESHB is the bottleneck of memory requirement for a simulation using TOUGH2-MP. By using a preprocessing program, the bottleneck for memory requirement can be avoided.

\section{PARAL.prm}

PARAL.prm is an optional file providing TOUGH2-MP some parameters. If this file does not exist in the working folder, the code will take default parameters. These parameters are needed if a user wants to try different options with the parallel linear solver, partitioning algorithms, and main program. The following is an example of the file.

1008680, 4000000, 0

AZ_solver AZ_bicgstab

AZ_scaling AZ_BJacobi

AZ_precond AZ_dom_decomp

AZ_tol 1.0e-6

AZ_overlap 0

AZ_max_iter 250

$A Z \_c o n v A Z \_r h s$

AZ_subdomain_solve $A Z \_i l u t$

AZ_output AZ_none

EE_partitioner METIS_Kway

EE_output 100

END OF INPUTS

The three numbers at first line are:

MNEL: $\quad$ Estimated total gridblocks, must be larger than model gridblock number. 
MCON: Estimated total connections, must be larger than model connection number.

PartReady: A parameter to inform the program that domain partitioning was done by a preprocessing program or will be done inside the TOUGH2-MP. If PartReady $=0$, the parallel code will perform domain partitioning during running the code. If PartReady $>0$, the code will not perform domain partitioning and partition data will be read directly from file "part.dat" at the working directory. Default PartReady=0.

The default values of MNEL and NCON are 500,000 and 2,300,000. The two parameters are required only in generating MESHA and MESHB and when a model has more than 500,000 gridblocks or $2,300,000$ connections.

From the second line and below, each line provides a parameter. These parameters give options or parameters for running the Aztec and METIS packages, and SAVE file output frequency control. The parameters can be in any order. If one parameter is not present, its default value will be used. Each line in the file consists of two terms. The first term is parameter's name and the second term is its value. Detailed content of the parameters is discussed below.

AZ_solver

AZ_cg

AZ_gmres

AZ_cgs

AZ_tfqmr

AZ_bicgstab

AZ_lu

Specifies solution algorithm, available solvers:

conjugate gradient (only applicable to symmetric positive definite matrices).

restarted generalized minimal residual.

conjugate gradient squared.

transpose-free quasi-minimal residual.

bi-conjugate gradient with stabilization.

sparse direct solver (single processor only).

AZ_scaling Specifies scaling algorithm, user can select from:

AZ_none no scaling.

AZ_Jacobi point Jacobi scaling.

AZ_BJacobi Block Jacobi scaling where the block size corresponds to the 
VBR blocks.

Az_row_sum scale each row so the magnitude of its elements sum to 1 .

AZ_sym_diag symmetric scaling so diagonal elements are 1 .

AZ_sym_row_sum symmetric scaling using the matrix row sums.

AZ_precond Specifies preconditioner. Available selections include:

AZ_none no preconditioning.

AZ_Jacobi $\quad \mathrm{k}$ step Jacobi (or block Jacobi for DVBR matrices)

AZ_Neumann Neumann series polynomial.

AZ_ls least-squares polynomial.

AZ_sym_GS non-overlapping domain decomposition (additive Schwarz) k step symmetric Gauss-Seidel.

AZ_dom_decomp domain decomposition preconditioner (additive Schwarz).

AZ_tol Specifies tolerance value used in conjunction with convergence tests.

AZ_type_overlap Determines how overlapping subdomain results are combined when different processors have computed different values for the same unknown.

AZ_standard the resulting value of an unknown is determined by the processor owning that unknown.

AZ_symmetric average the results obtained from different processors corresponding to the same unknown.

AZ_overlap Determines the submatrices factored with the domain decomposition algorithms.

AZ_max_iter Maximum number of iterations.

AZ_conv Determines the residual expression used in convergence check and printing. Available selections include: AZ_r0, AZ_rhs, AZ_Anorm, AZ_noscaled, AZ_sol, AZ_weighted.

AZ_subdomain_solve Specifies the solver to use on each subdomain when AZ_precond is set to AZ_dom_decomp, available selections include: AZ_lu, AZ_ilut, AZ_ilu, AZ_rilu, AZ_bilu, and AZ_icc.

AZ_reorder Determines whether RCM reordering will be done in conjunction with domain decomposition incomplete factorizations, 1 yes; 0 no. 

AZ_pre_calc
Indicates whether to use factorization information from previous
calls to AZ_solve, three selections: AZ_calc, AZ_recalc, and AZ_reuse.
AZ_output Specifies information to be printed, available selections: AZ_all, AZ_none, AZ_warnings, AZ_last, and $>0$.
EE_partitioner Specifies the partitioner to be used, user can select partitioners from: METIS_Kway uses the multilevel $k$-way partitioning algorithm. The objective of this partitioning method is to minimize the edgecut. It should be used to partition a graph into a large METIS_Vkway uses the multilevel $k$-way partitioning algorithm. The objective of this partitioning method is to minimize the total communication volume.
METIS_Recursive uses multilevel recursive bisection. The objective of this partitioning method is to minimize the edgecut, this function should be used to partition a graph into a small number of partitions (less than 8).
EE_output Output control for solution results. The SAVE file will be written every EE_output time steps. If EE_output=0, no SAVE file will be written out until last time step. A special value of 666888 for this parameter will evoke debugging run, which will produce more informative output.

More options or parameters for the Aztec parallel linear equation solver can be specified. For further discussion, readers may refer to Tuminaro et al. (1999). Table 4-2 presents the default values used in TOUGH2-MP. 
Table 4-2. Default values of the options and parameters

\begin{tabular}{|l|l|}
\hline Parameters or options & Values \\
\hline AZ_solver & AZ_bicgstab \\
\hline AZ_scaling & AZ_Bjacobi \\
\hline AZ_pecond & AZ_dom_decomp \\
\hline AZ_tol & $1 \times 10^{-6}$ \\
\hline AZ_type_overlap & AZ_standard \\
\hline AZ_max_iter & 500 \\
\hline AZ_conv & AZ_r0 \\
\hline AZ_subdomain_solve & AZ_ilut \\
\hline AZ_reorder & 1 \\
\hline AZ_pre_calc & AZ_calc \\
\hline AZ_output & AZ_none \\
\hline EE_partitioner & METIS_Kway \\
\hline EE_output & 200 \\
\hline
\end{tabular}

\section{INCON}

During initialization of a TOUGH2 run, all gridblocks are first assigned to the default thermodynamic conditions specified in data block PARAM in file INFILE. The default initial conditions may be superseded by thermodynamic conditions assigned to individual gridblocks in disk file INCON. File format of INCON is the same as in the serial version of the TOUGH2 code.

The INCON file is set up either by user or generated by a previous TOUGH2 run through an output file SAVE (compatible with formats of file or data block INCON for initializing a continuation run). The INCON file can be obtained by simply renaming SAVE file to INCON. If INCON file is set up by the user, only a fraction of the grid blocks may be specified or the blocks may be in a random sequence. Accordingly, TOUGH2-MP will perform a gridblock index search first. 


\section{GENER}

The format of file GENER is the same as the block format described in Section 4.2.

\section{part.dat}

If parameter PartReady in "PARAL.prm" has a value larger than 0, the parallel code will read file "part.dat" from working directory during run-time. The file contains domainpartitioning results. It is read by the following code:

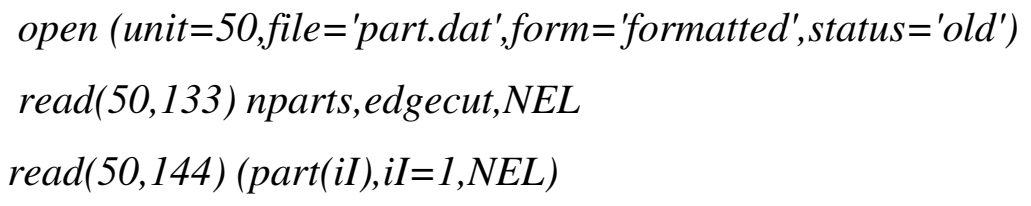

where

nparts Number of portions that the domain has been partitioned into. It must equal to the number of processors/processes used for solving the problem.

edgecut Number of cut edges.

nel Total number of elements or gridblocks in the domain.

part Partitioning result of each gridblock. The integer value indicates that the gridblock is in which processor.

File "part.dat" can be created through a preprocessing program based on user's special requirements, e.g. based on physical boundaries of modeling domain for grid partitioning.

\section{flow9.dat, flow9b.dat}

File flow9.dat is required only by the T2R3D module. When running T2R3D for a tracer or contaminant transport simulation, the file, flow9.dat, will provide flow field information. This file is generated by flow simulation (EOS9 module) with option $\operatorname{mop}(17)=1$. File flow $9 \mathrm{~b}$.dat is an intermediate file which is created by TOUGH2-MP at its first run. The file is in binary format with the same contents as flow9.dat. If flow9b.dat 
exists in the working directory, the code will ignore the flow9.dat file and read information directly from flow9b.dat. Once the flow9.dat is changed, flow 9 b.dat must be deleted from the working folder to make the changes take effect. The purpose of using an intermediate file flow9b.dat is to reduce the memory requirement for the master processor and to enhance I/O efficiency.

File flow $9 \mathrm{~b} . d a t$ is saved in the following sequence:

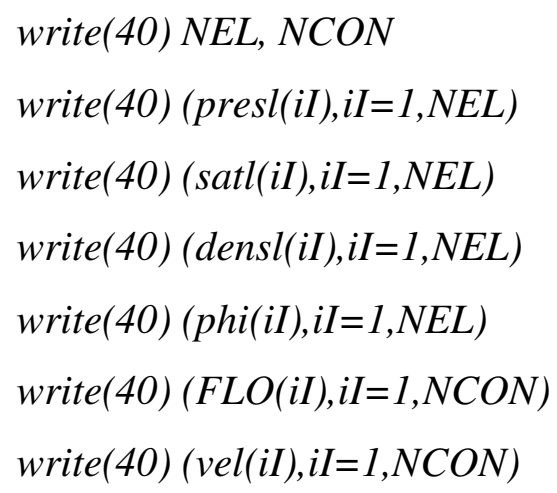

\subsection{Output from TOUGH2-MP}

TOUGH2-MP produces a variety of output, most of which can be controlled by the user. Information written in the initialization phase on to the standard output file includes parameter settings in the main program for dimensioning of problem-size dependent 
arrays, and disk files in use. This is followed by documentation on settings of the MOPparameters for choosing program options, and on the EOS-module. During execution, the parallel program can optionally generate a brief message for Newtonian iterations and time steps. At the end, a summary of subroutines used and parallel computation information are provided. In TOUGH2-MP, standard output at user-specified simulation times or time steps is generated by a subroutine called FINALOUT, which is applied to replace the OUT subroutine in TOUGH2 V1.4/V2.0. Each EOS module comes with its own routine FINALOUT. The output file for TOUGH2 is replaced by two files in TOUGH2-MP, named OUTPUT and OUTPUT_DATA. The first file provides problem initialization, time-stepping, and parallel computing information, and the second file gives a complete element-by-element and/or connection-by-connection report of thermodynamic state variables and other important parameters. The sequence for outputs of gridblocks and connections is not in the original sequence as listed in MESH file. It is written out processor-by-processor depending on the domain partition results. In addition, there are some minor differences in naming conventions used by different EOS modules. 


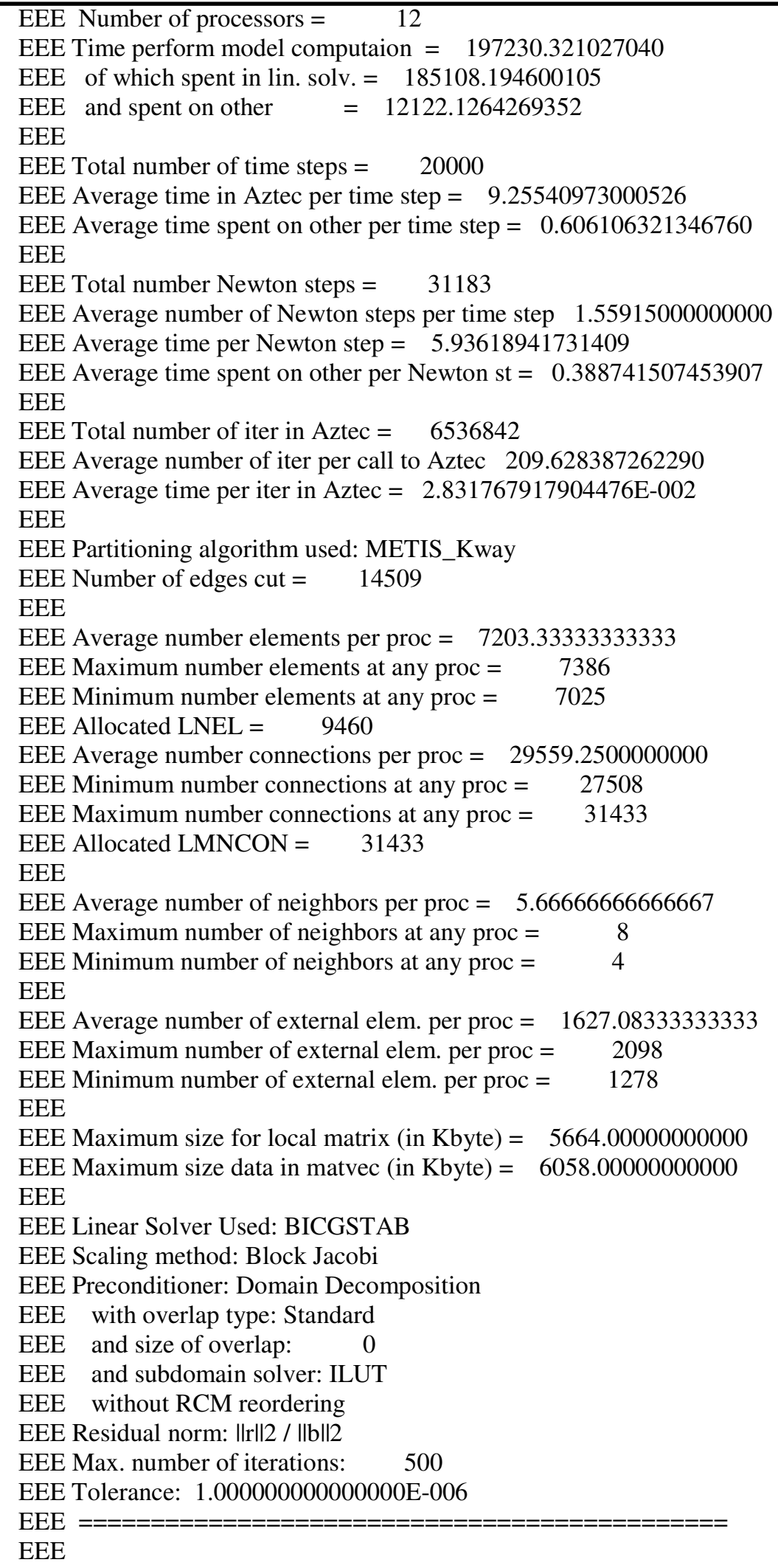

Figure 4-1 Example for output of parallel computing information 
The parallel computing information which is new to original TOUGH2 outputs is written out near the end of OUTPUT file. Figure 4-1 shows an example of a portion of the output. The output provides detailed information of the number of processors used, timing for tasks, code performance for each time step, Newton iteration, and linear iteration, algorithm used for domain partitioning, and domain decomposition results. At the end of the list in Figure 4-1, linear solver, preconditioner, and options and parameters selected for solving the linear equations are presented. This information is very important for evaluating the parallel code performance.

Some informative output generated by other than master processor is written to fort.36. The user may get additional information for the program run from this file. Other output files include SAVE and FOFT_P.xxx. The SAVE contains primary variables for a continuation run, and has the same format as INCON. The requested time-dependent data for gridblocks (identified with FOFT), connections (COFT), and source/sinks (GOFT) are written out to file FOFT_P.xxx. The extension name $\mathrm{xxx}$ is a number indicating the processor number by which the file is written. TOUGH2-MP can generate multiple time-dependent data output from different processors.

Main output parameters are summarized in alphabetical order in Table 4-3.

Table 4-3. TOUGH2 Output variables and their definition

\begin{tabular}{|l|l|}
\hline DELTEX & Time step size, seconds \\
\hline DG & Gas phase density, $\mathrm{kg} / \mathrm{m}^{3}$ \\
\hline DL & Liquid (aqueous phase) density, $\mathrm{kg} / \mathrm{m}^{3}$ \\
\hline DT & Time step size, seconds \\
\hline DW & Water (aqueous phase) density, $\mathrm{kg} / \mathrm{m}^{3}$ \\
\hline DX1, DX2, etc. & Changes in first, second, etc. thermodynamic variable \\
\hline DX1M, DX2M, DX3M & $\begin{array}{l}\text { Maximum change in first, second, and third primary variable } \\
\text { In current time step }\end{array}$ \\
\hline ELEM & Code name of element \\
\hline ELEM1, ELEM2 & $\begin{array}{l}\text { Code name of first and second element, respectively, in a } \\
\text { flow connection }\end{array}$ \\
\hline ENTHALPY & Flowing specific enthalpy for mass sinks/sources, J/kg \\
\hline FF(GAS), FF(LIQ) & $\begin{array}{l}\text { Mass fraction of flow in gas and liquid phases, respectively } \\
\text { (mass production wells only) }\end{array}$ \\
\hline
\end{tabular}




\begin{tabular}{|c|c|}
\hline FLO(BRINE) & $\begin{array}{l}\text { Total rate of brine flow, } \mathrm{kg} / \mathrm{s} \text { (positive if from ELEM2 into } \\
\text { ELEM1) }\end{array}$ \\
\hline FLOF & $\begin{array}{l}\text { Total rate of fluid flow, } \mathrm{kg} / \mathrm{s} \text { (positive if from ELEM2 into } \\
\text { ELEM1) }\end{array}$ \\
\hline FLO(GAS) & $\begin{array}{l}\text { Total rate of gas flow, } \mathrm{kg} / \mathrm{s} \text { (positive if from ELEM2 into } \\
\text { ELEM1) }\end{array}$ \\
\hline FLOH & $\begin{array}{l}\text { Total rate of heat flow, W (positive if from ELEM2 into } \\
\text { ELEM1) }\end{array}$ \\
\hline FLO(LIQ) & $\begin{array}{l}\text { Total rate of liquid (aqueous phase) flow, } \mathrm{kg} / \mathrm{s} \text { (positive if } \\
\text { from ELEM2 into ELEM1) }\end{array}$ \\
\hline GENERATION RATE & Sink $(>0)$ or source $(<0)$ rate, $\mathrm{kg} / \mathrm{s}$ (mass), W (heat) \\
\hline INDEX & $\begin{array}{l}\text { Internal indexing number of elements, connections, } \\
\text { sinks/sources }\end{array}$ \\
\hline ITER & Number of Newtonian iterations in current time step \\
\hline ITERC & $\begin{array}{l}\text { Total cumulative number of Newtonian iterations in } \\
\text { simulation Run }\end{array}$ \\
\hline KCYC & Time step counter \\
\hline KER & Index number of equation with largest residual \\
\hline $\mathrm{K}(\mathrm{GAS})$ & Gas phase relative permeability \\
\hline K(LIQ) & Liquid (aqueous) phase relative permeability \\
\hline KON & $\begin{array}{l}\text { Convergence flag; KON = 2: converged; KON = 1: not } \\
\text { Converged }\end{array}$ \\
\hline MAX. RES. & $\begin{array}{l}\text { Maximum (relative) residual in any of the mass and energy } \\
\text { Balance equations (see Equation B.8) }\end{array}$ \\
\hline NER & Index number of element (gridblock) with largest residual \\
\hline $\mathrm{P}$ & Pressure, $\mathrm{Pa}$ \\
\hline PER.MOD. & Permeability modification coefficient \\
\hline PCAP & Capillary pressure, $\mathrm{Pa}$ \\
\hline PSAT & Saturated vapor pressure, $\mathrm{Pa}$ \\
\hline $\mathrm{P}(\mathrm{WB})$ & $\begin{array}{l}\text { Flowing bottomhole pressure (production wells on } \\
\text { deliverability only), Pa }\end{array}$ \\
\hline RL & Relative humidity \\
\hline SG & Gas saturation \\
\hline SL & Liquid saturation \\
\hline SOURCE & Code name of sink/source \\
\hline ST & Simulation time, in seconds \\
\hline
\end{tabular}




\begin{tabular}{|l|l|}
\hline SW & Water (aqueous phase) saturation \\
\hline $\mathrm{T}$ & Temperature, ${ }^{\circ} \mathrm{C}$ \\
\hline TOTAL TIME & Simulation time, in seconds \\
\hline VEL(GAS) & $\begin{array}{l}\text { Gas phase pore velocity, } \mathrm{m} / \mathrm{s} \text { (positive if from ELEM2 into } \\
\text { ELEM1) }\end{array}$ \\
\hline VEL(LIQ) & $\begin{array}{l}\text { Liquid (aqueous) phase pore velocity, } \mathrm{m} / \mathrm{s} \text { (positive if from } \\
\text { ELEM2 into ELEM1) }\end{array}$ \\
\hline VIS(LIQ) & Liquid (aqueous) phase viscosity, Pa-s \\
\hline X1, X2, etc. & $\begin{array}{l}\text { First, second, etc. thermodynamic variable (also: water 1, } \\
\text { water 2) }\end{array}$ \\
\hline XAIRG & Mass fraction of air in gas phase \\
\hline XAIRL & Mass fraction of air in liquid phase \\
\hline
\end{tabular}

\section{USER FEATURES}

TOUGH2-MP possesses features from both TOUGH2 V2.0 and V1.4. Some additional useful functions have also been implemented into the parallel code. The most important features include:

- Dynamic memory allocation. The program allocates arrays according to the problem size: total number of grid blocks, connections, materials, and source/sinks terms. Different from the V2.0 and V1.4 versions, the code does not need recompilation for different size problems. In addition, the code does not have limitations on the number of materials.

- Block-by-block permeability modification. Through this function, heterogeneous flow systems may be specified by providing gridblocks with different hydrogeologic properties through applying permeability modification (PM) coefficients to individual gridblocks. To be consistent with the V2.0, the random numbers are generated by single processor and then distributed to all processors involved in the computation.

- Initial and boundary conditions. TOUGH2-MP is fully compatible with V2.0 andV1.4 for initial and boundary conditions. The parallel code allows time 
dependent first-type (Dirichlet) boundary conditions (see TIMBC input keyword). Internally, it always uses large-volume approach for handling the first type boundary conditions.

- Flow in fractured media. TOUGH2-MP retains the full functions of TOUGH2 for a flexible description of flow in fractured media through either discrete fracture, dual-porosity, dual-permeability, or MINC approaches. In addition, the parallel code allows several schemes of fracture-matrix interface area reduction for F-M local connection and mobility weighting, such as, using active fracture model. These schemes are invoked through setting a negative integer for parameter ISOT.

- Efficient computing schemes. In addition to the parallel computing approach, TOUGH2-MP also adopts very efficient algorithms for index searching and other computations. In the sequential version, index searching is a time-consuming task. In the parallel version, index searching can be done in seconds even for multimillion gridblock problems.

- Other features. TOUGH2-MP allows more time steps at each simulation run (TOUGH2 limits to 9999), can control time step-size reduction or increase depending on rate of convergence, allows gridblock names with 5 or 8 characters, allows generating multi-million gridblocks for Cartesian X-Y-Z mesh maker, and includes more relative permeability and capillary functions.

\section{SAMPLE PROBLEMS}

Several sample problems are included in the TOUGH2-MP distribution package. Users may use these examples as benchmarks for proper code installation and for testing the code's performance on their computers. In addition, the input data files can also be used as templates to facilitate preparation of input data for new simulations. Actually, any input data files for the TOUGH2 V2.0 and V1.4 can be used as TOUGH2-MP input without change (need renaming the input file name to "INFILE"). The input and output files for the sample problems can also be obtained from following website: http://www.tough2.com/examples.html. 


\subsection{Unsaturated Flow Simulation}

Successfully running this small test problem indicates correct installation of TOUGH2MP. This example also verifies TOUGH2-MP for using a different number of processors. The parallel code running on a different number of processors and the sequential version of the code should yield results within the acceptance criteria. The problem size is too small to demonstrate speed-up of parallel simulation.

This problem concerns one-dimensional vertical flow through a single vertical column of highly heterogeneous dual-permeability fractured medium. The column is abstracted from a three-dimensional Yucca Mountain site-scale model. The computational mesh consists of 136 elements and 205 connections. The bottom boundary is treated as a Dirichlet-type boundary. Constant liquid saturation is provided at the bottom boundary by specifying a large-volume for the bottom gridblock. A source term with a rate of $0.4765 \mathrm{E}-04 \mathrm{~kg} / \mathrm{s}$ is introduced to the second fracture element from the top to provide a constant water infiltration. Simulations are run using 2,4 , and 8 processors by TOUGH2MP. The EOS9 module is used for the test problem simulation.

Table 6-1 Comparison of simulated liquid saturation by TOUGH2-MP and TOUGH2 Version 1.4 at the time of 1000 year for randomly selected 10 gridblocks

\begin{tabular}{|l|r|r|r|r|}
\hline \multirow{2}{*}{ Elements } & \multicolumn{4}{|c|}{ Simulated liquid saturation } \\
\cline { 2 - 5 } & $\begin{array}{c}\text { By TOUGH2 } \\
\text { V1.4 }\end{array}$ & \multicolumn{3}{|c|}{ By TOUGH2-MP } \\
\cline { 3 - 5 } & 2 processors & 4 processors & 8 processors \\
\hline Faa61 & $6.2713 \mathrm{E}-02$ & $6.2713 \mathrm{E}-02$ & $6.2713 \mathrm{E}-02$ & $6.2713 \mathrm{E}-02$ \\
\hline Maa61 & $5.2041 \mathrm{E}-01$ & $5.1926 \mathrm{E}-01$ & $5.1909 \mathrm{E}-01$ & $5.1899 \mathrm{E}-01$ \\
\hline Fja61 & $8.9186 \mathrm{E}-02$ & $8.9186 \mathrm{E}-02$ & $8.9186 \mathrm{E}-02$ & $8.9186 \mathrm{E}-02$ \\
\hline Mja61 & $9.9892 \mathrm{E}-01$ & $9.9892 \mathrm{E}-01$ & $9.9892 \mathrm{E}-01$ & $9.9892 \mathrm{E}-01$ \\
\hline Fpa61 & $3.4302 \mathrm{E}-02$ & $3.4286 \mathrm{E}-02$ & $3.4286 \mathrm{E}-02$ & $3.4284 \mathrm{E}-02$ \\
\hline Mpa61 & $5.3516 \mathrm{E}-01$ & $5.3502 \mathrm{E}-01$ & $5.3502 \mathrm{E}-01$ & $5.3500 \mathrm{E}-01$ \\
\hline Fua61 & $8.1080 \mathrm{E}-02$ & $8.1024 \mathrm{E}-02$ & $8.1014 \mathrm{E}-02$ & $8.1008 \mathrm{E}-02$ \\
\hline Mua61 & $6.5629 \mathrm{E}-01$ & $6.5571 \mathrm{E}-01$ & $6.5562 \mathrm{E}-01$ & $6.5556 \mathrm{E}-01$ \\
\hline F(a61 & $3.1185 \mathrm{E}-01$ & $3.1107 \mathrm{E}-01$ & $3.1094 \mathrm{E}-01$ & $3.1087 \mathrm{E}-01$ \\
\hline M(a61 & $9.8998 \mathrm{E}-01$ & $9.8998 \mathrm{E}-01$ & $9.8998 \mathrm{E}-01$ & $9.8998 \mathrm{E}-01$ \\
\hline
\end{tabular}

Table 6-1 shows the excerpts of the comparison of simulated saturation by TOUGH2-MP and TOUGH2 at 10 randomly selected gridblocks. Figure 6-1 shows the comparison of simulated pressure in fractured continuum. The comparisons demonstrate that simulation results from the two codes match very well. 
Simulated Pressure in Fracture Continuum at Time 1000 Year

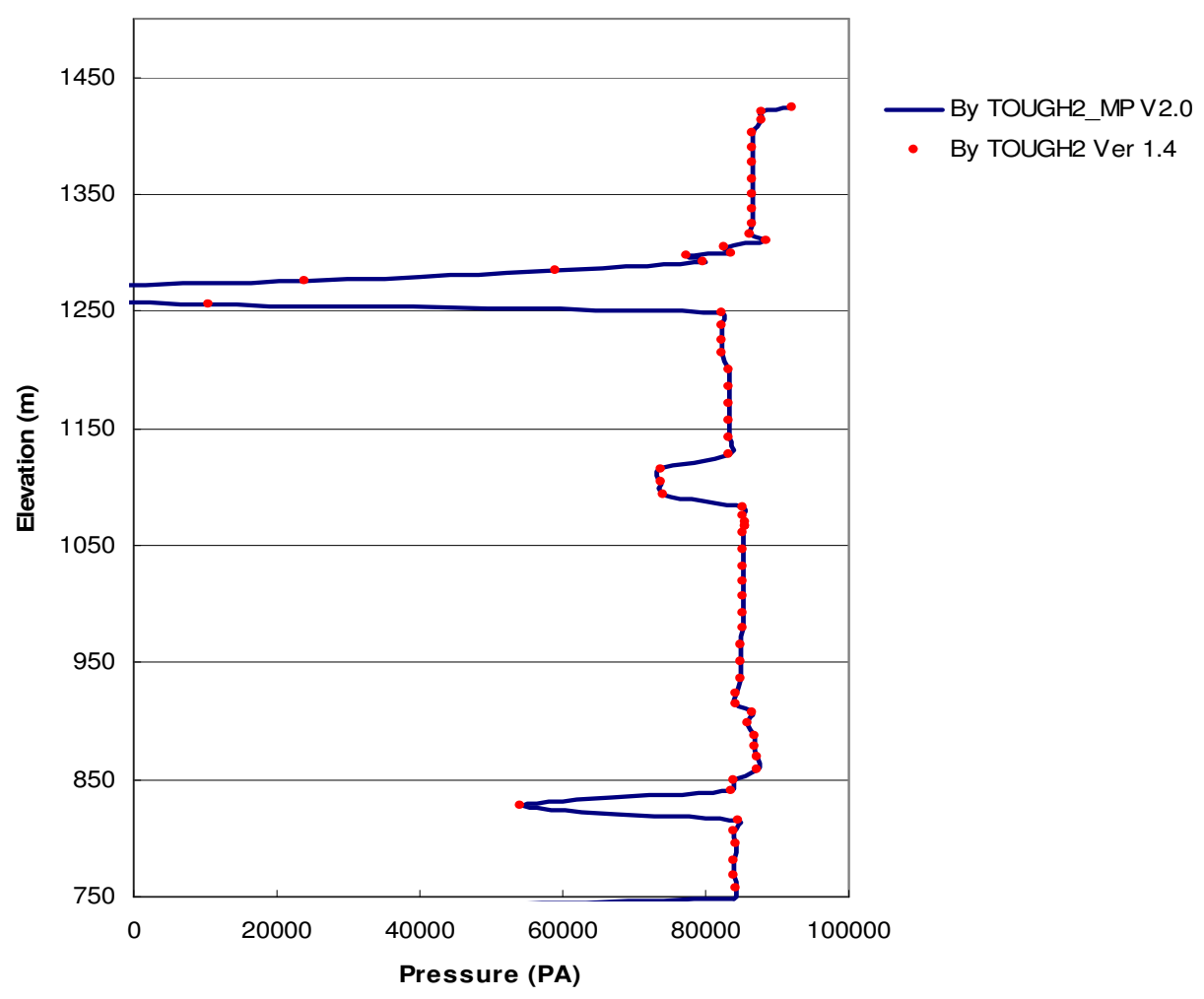

Figure 6-1. Comparison of simulated pressure in fracture continuum by TOUGH2MP and TOUGH2 Version 1.4. The figure shows simulation result from 2 processors. Simulation results by different number of processors are almost identical.

\subsection{Contaminant Transport Simulation}

This example is adopted from a model for investigation of flow focusing and discrete flow paths in the Topopah Spring welded (TSw) hydrogeologic unit at the Yucca Mountain site (Bodvarsson et al., 2003). In this model, tracer transport is simulated through the fracture network to demonstrate degree of preferential flow. It is designed to test the T2R3D module for modeling of contaminant transport in a fracture continuum.

The two-dimensional vertical cross section for this problem has an upper boundary at the bottom of the Paintbrush Tuff nonwelded (PTn) unit and a lower boundary at the proposed repository zone. The cross section is $100 \mathrm{~m}$ wide and $150 \mathrm{~m}$ high, and is discretized in a uniform 2-D grid of $\Delta \mathrm{x}=0.25 \mathrm{~m}$ and $\Delta \mathrm{z}=0.5$, resulting in 120,000 
gridblocks and 239,300 connections. The $150 \mathrm{~m}$ vertical extent of the model corresponds to the average distance from the top of the $\mathrm{TSw}$ to the top of the proposed repository horizon over the repository area.

The flow field used for contaminant transport simulation is given by file flow9.dat, which was generated by flow model (EOS9 module with option MOP17=1) with following specification: Uniform percolation flux $(5 \mathrm{~mm} / \mathrm{year})$ boundary conditions are prescribed at the upper boundary. The two side boundaries are treated as no-flow boundaries; the bottom boundary allows gravitational drainage out of the model. The fractured rock is modeled using a randomly distributed permeability field to represent the complex fracture distributions. Fracture permeability is prescribed stochastically, based on measured air permeability data. The steady state results of the flow system are used as input for contaminant transport simulation. Detailed discussion of the flow simulation was presented in Bodvarsson et al. (2003).

A conservative, nonsorbing tracer with a molecular diffusion coefficient of $3.2 \times 10^{-11} \mathrm{~m}^{2} / \mathrm{s}$ at constant concentration with a mass fraction of 0.1 is prescribed at the top boundary. Under steady-state flow condition, the tracer is transported into the model domain from the top by advection and diffusion. Simulation of the contaminant transport processes is relatively easy, because the problem involves only a linear process. The simulations can be completed in a few seconds. We have run the model with 2, 4, and 8 CPUs and noticed the code performs extremely well in speedup (linear or super-linear speedup can be seen).

Figure 6-2 shows the simulation results of mass fraction distribution by TOUGH2-MP at time 1 year, which is identical to the results from T2R3D (DC) Version 1.4. 


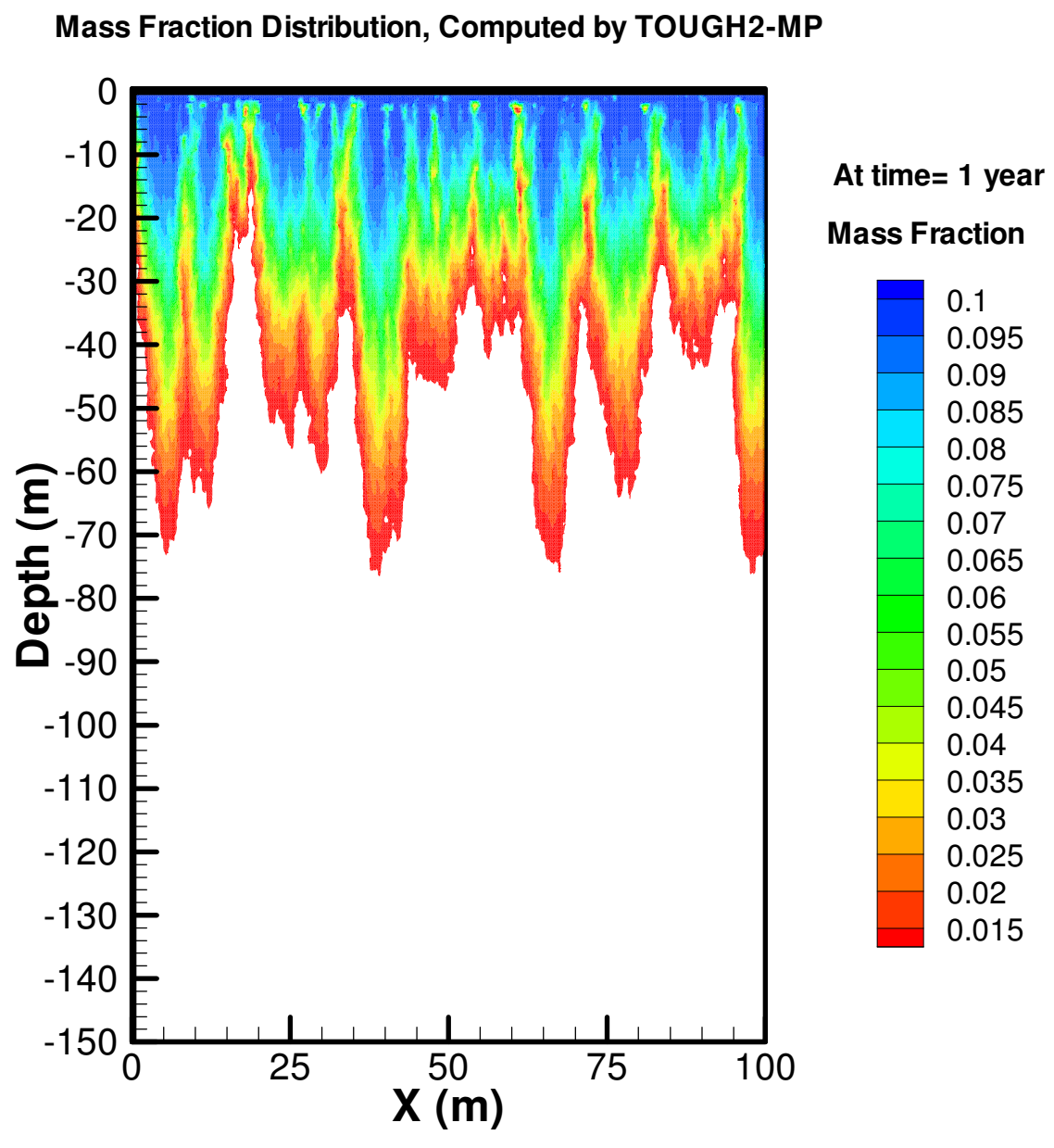

Figure 6-2. Simulated mass fraction distribution after 1 year for TOUGH2-MP sample problem 2.

\subsection{Investigation of $\mathrm{CO}_{2}$ Convection Mixing}

This sample problem was modified from a previously published paper on modeling studies of $\mathrm{CO}_{2}$ sequestration in saline aquifers (Zhang et al., 2007). The example shows performance of TOUGH2-MP (ECO2N module) on large-scale simulations, which are too large to be run with the sequential version TOUGH2 code.

When $\mathrm{CO}_{2}$ is injected into a saline formation, it partially displaces the resident brine, and partially dissolves in it, while some water also dissolves (evaporates) into the flowing $\mathrm{CO}_{2}$ stream. Under most subsurface temperature and pressure conditions, $\mathrm{CO}_{2}$ is buoyant (less dense), compared to water (or brine), and the injected $\mathrm{CO}_{2}$ will move upward towards the top of the permeable interval. Eventually, the carbon dioxide is distributed 
among mobile layers beneath the caprock. When $\mathrm{CO}_{2}$ dissolves in brine, the density of the aqueous phase will increase by a small amount of approximately $1 \%$. Although small, the density increase is sufficient to trigger convection flow, provided there is "sufficient" vertical permeability. This example presents the simulation of the convection processes.

$\mathrm{CO}_{2}$ convection starts slowly and within a small space scale, which over time grows to larger-scale flows. We are using a three-dimensional high-resolution model to begin a systematic evaluation of the role of brine convection in enhancing $\mathrm{CO}_{2}$ dissolution. Our initial studies used a cube of $1 \mathrm{~m} \times 1 \mathrm{~m} \times 1 \mathrm{~m}$ size to investigate the onset and early stage of $\mathrm{CO}_{2}$ convection. Fine gridding $(\Delta \mathrm{x}=1 \mathrm{~cm})$ is used for representing the interplay between molecular diffusion and aqueous phase convection induced by the small density change due to $\mathrm{CO}_{2}$ dissolution, resulting in $100 \times 100 \times 100=1,000,000$ gridblocks. With an additional 10,000 top boundary gridblocks, the model includes 1,010,000 gridblocks and $2,999,800$ connections between them.

Model initial and boundary conditions are shown in Figure 6-3. Two-phase conditions with a free $\mathrm{CO}_{2}$-rich phase (gas saturation $\mathrm{S}_{\mathrm{g}}=0.1 \%$ ) are maintained at the upper boundary. Conditions of no fluid flow at the upper boundary are enforced by specifying a very small permeability in the boundary domain $\left(10^{-50} \mathrm{~m}^{2}\right)$. This results in mass transport across the top boundary occurring by molecular diffusion only. The model domain is assigned an isotropic permeability of $10^{-11} \mathrm{~m}^{2}$. Permeabilities for all gridblocks are modified by multiplying the assigned permeability with a random number in the range 0.99-1.01. This small modification is applied to trigger the onset of $\mathrm{CO}_{2}$ convection.

The flow domain is initialized with a constant pressure, but because of the small compressibility of the aqueous phase, hydrostatic pressure equilibrium is established virtually instantaneously. $\mathrm{CO}_{2}$ then diffuses into the initially $\mathrm{CO}_{2}$-free aqueous phase below, causing brine density to increase and eventually triggering downward advection. The unstable nature of the advection (denser fluid above less-dense fluid) gives rise to fingering, see Figure 6-4. The figure shows $\mathrm{CO}_{2}$ mass fractions at a time of 20.24 days at 
$\mathrm{y}=0.505 \mathrm{~m}$, which represents a vertical cross-section located near the center of the cube, and it demonstrates convective fingering in brine with $12.5 \%$ salinity.

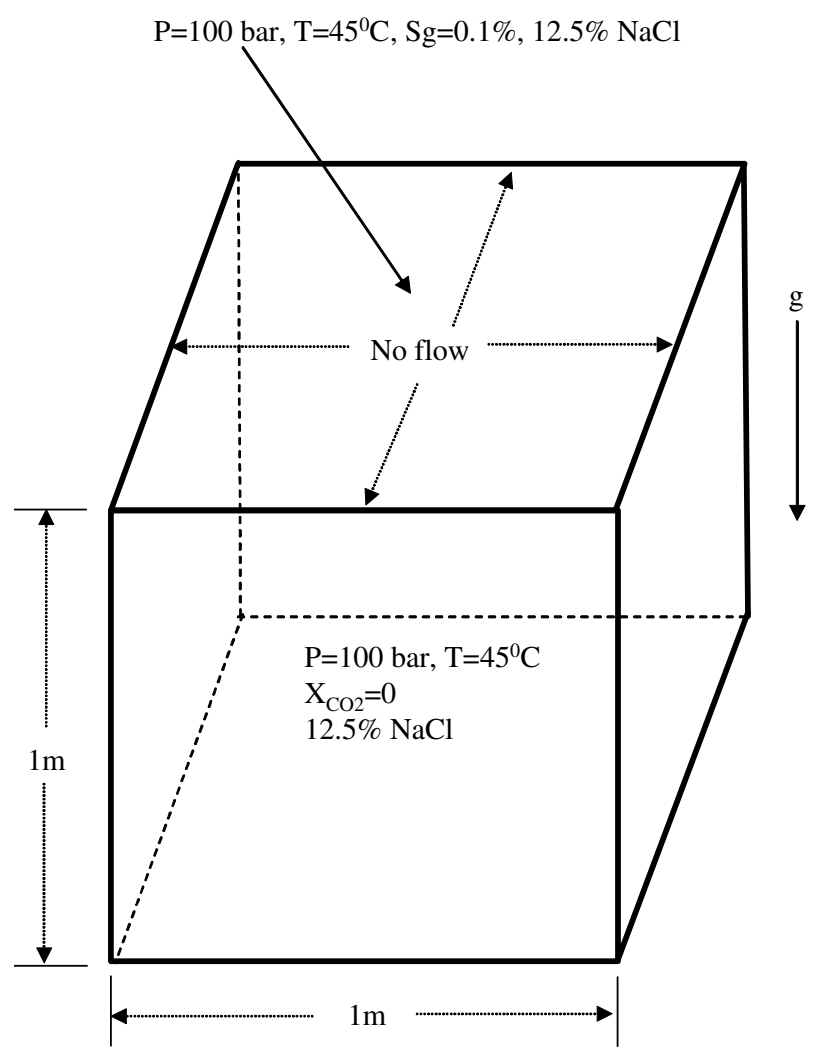

Figure 6-3. Three-dimensional domain for simulating brine convection induced by $\mathrm{CO}_{2}$ dissolution and associated increase in aqueous phase density. Initial and boundary conditions are also shown.

Simulations were run on a Linux cluster equipped with 356 nodes using infiniBand switch connection, and each node consists of 2 Opteron $2.2 \mathrm{GHz}$ CPUs. For testing the parallel code performance, the model was run using either $2,4,8,16,32,64,128$, or 256 processors for the same simulation time period. Figure 6-5 shows the speedups obtained for different numbers of processors and for different parts of the simulation. By increasing the number of processors, the total execution time was reduced from 79,160 seconds using two processors to 514 seconds using 256 processors. The parallel code demonstrates much better performance than ideal linear speedup. The total execution time is reduced to less than half when doubling the processor numbers when using 64 or less processors for this problem. Figure 6-5 indicates that the super-linear effect is introduced by the linear equation solution. It is interesting to note that this large-scale 
problem can be run on two processors, because of the huge memory available for each node (2 CPUs share 6 GB memory).

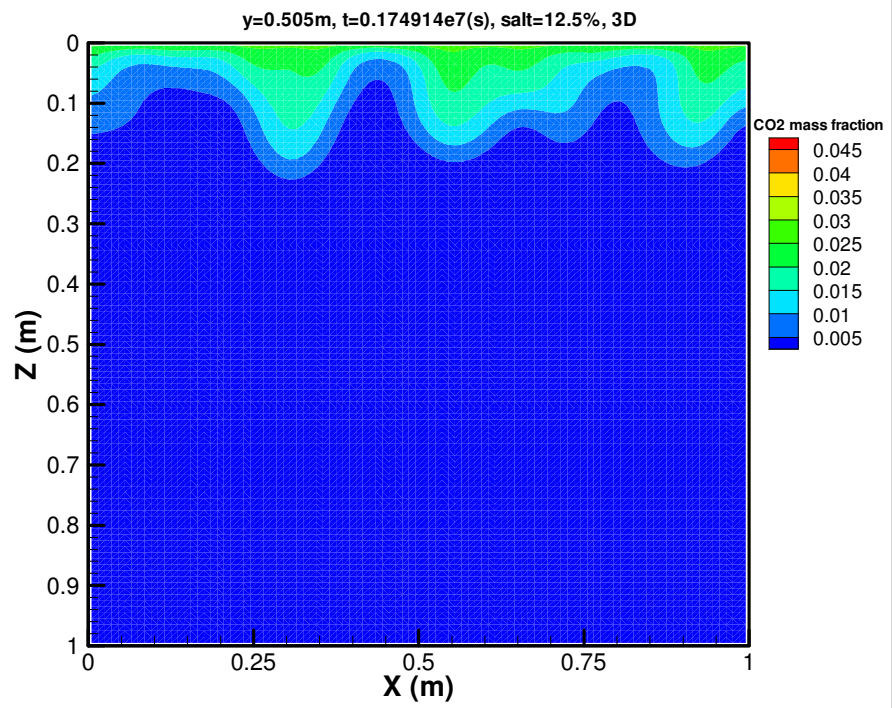

Figure 6-4 $\mathrm{CO}_{2}$ mass fraction distribution along cross-section $\mathrm{y}=0.505 \mathrm{~m}$ at time 20.2 days. The brine has 12.5 weight- $\% \mathrm{NaCl}$.

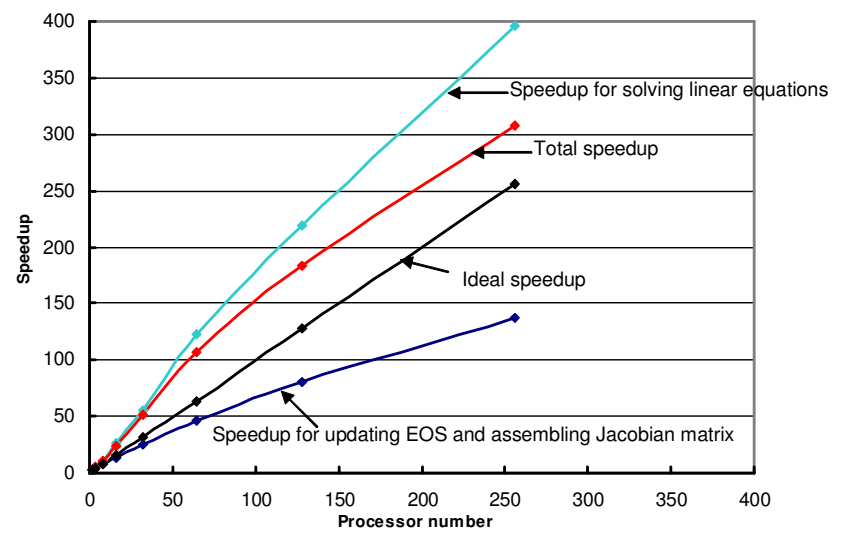

Figure 6-5. Speedups for the different parts of the parallel simulations.

\subsection{Large-scale two-phase water and hydrogen flow simulation}

One of the major problems in representing gas migration in a repository for radioactive waste is to model simultaneously all gas sources and the transfer pathways constituted by the network of undergrounds drift. The gas sources include significant quantities of hydrogen generated by the corrosion of metal components. In 2006, the French National Agency for Radioactive Waste Management (ANDRA) launched a multi-phase flow 
simulation benchmark exercise, named Couplex-Gaz, for modeling such a two-phase flow system (see http://www.andra.fr/interne.php3?id_article=913\&id_rubrique=76). This example is adopted from the test case 2 of the exercise completed by the first author of this manual and AF-Colenco Ltd, Switzerland (Croisé and Zhang, 2008).

The model represents a fraction of a repository with vitrified waste consisting of two rows of 30 waste cells. The three dimensional definition of the domain is shown in Figures 6-6 to 6-8. The vertical extension is limited to the thickness of a single indurate clay formation (Callovo-Oxfordian). The extensions in both other directions are representative of the distances between disposal cells. Detailed description of the model and model parameters are given in an ANDRA's report at: http://www.andra.fr/IMG/pdf/test_cases2.pdf.

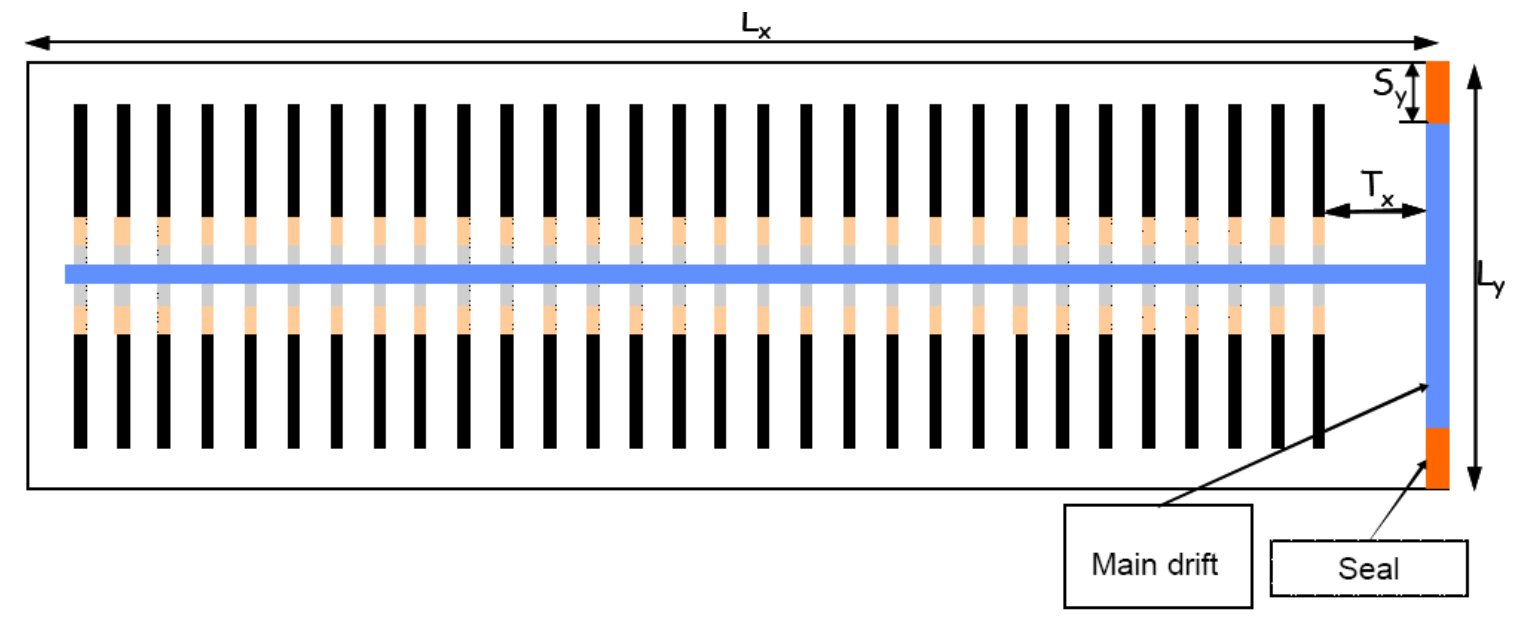

Figure 6-6 Representation of the domain in the horizontal plane XY (from Andra, 2006). Due to symmetry along $X$ axis, only half of the waste cells are simulated in the model. 


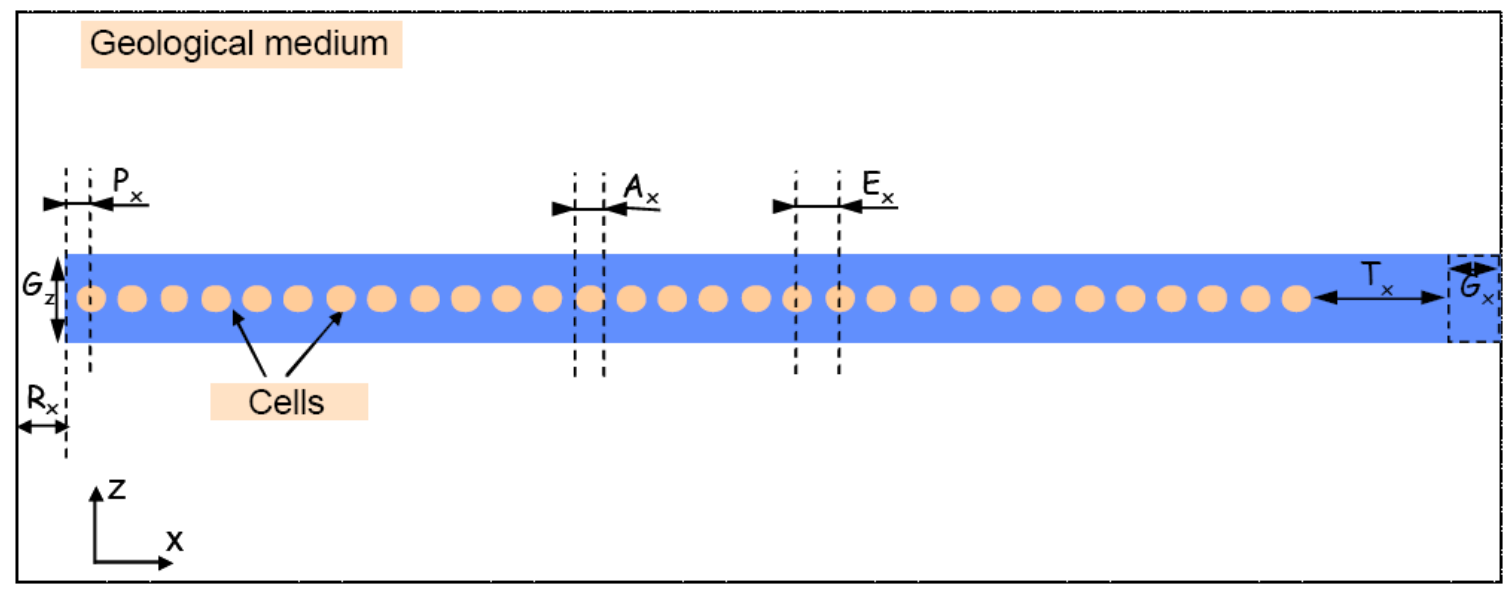

Figure 6-7 Model domain in the vertical plane XZ (from Andra, 2006).

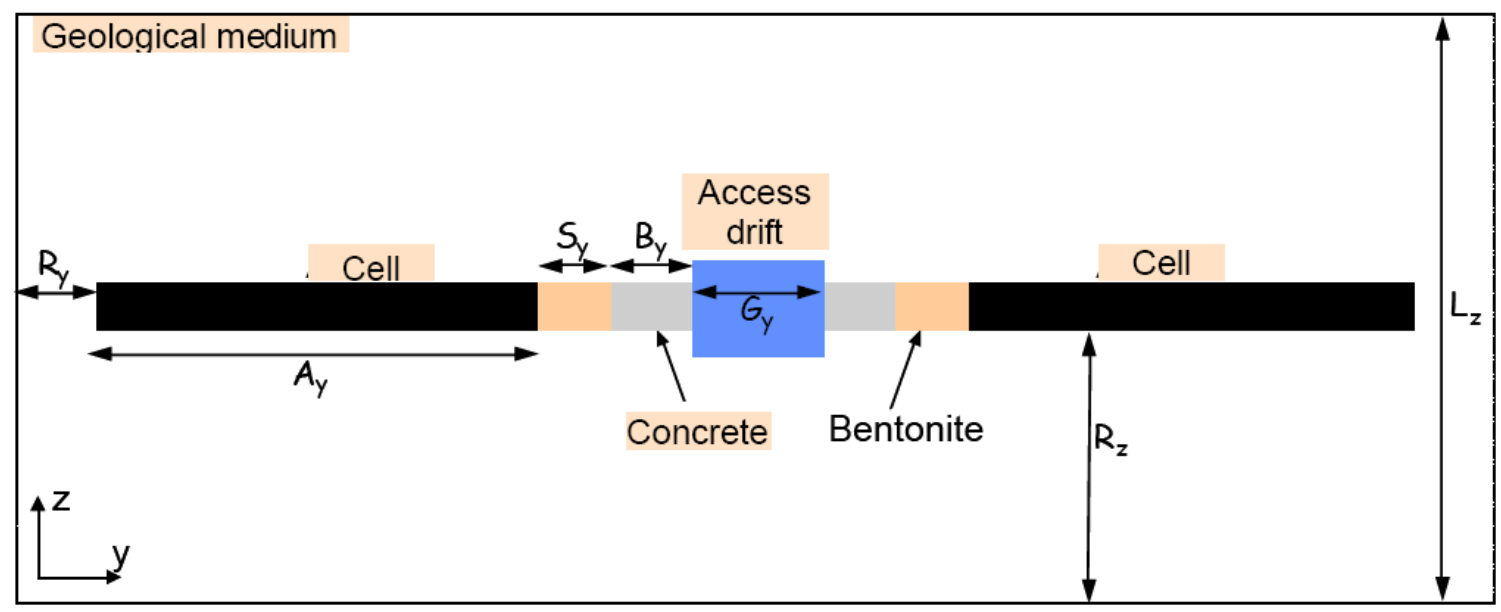

Figure 6-8 Model domain in the vertical planeYZ (from ANDRA, 2006).

Gas is generated by each disposal cell. Gas source term is imposed on the external surface of the cylinder that represents schematically the cell. The materials to be taken into account in that simulation include the backfill of the drift, the bentonite of the drift seals and the Callovo-Oxfordian formation. The cell is constituted of a material impermeable to both water and gas. Table 6-3 provides physical parameters for the materials of the model.

Based on the definition of the three-dimensional geometry (Table 6-2) for the domain, a 3D mesh representing model domain was generated using WinGridder Ver 4.0 (Pan 2008). Figures 6-9 to 6-11 show the cross-section of the 3D mesh along different planes. The mesh consists of 62,401 gridblocks and 184,260 connections. 
Table 6-2 Size of items in the calculation domain (From ANDRA, 2006)

\begin{tabular}{|l|c|c|}
\hline \multicolumn{1}{|c|}{ Name of parameter } & Parameter & Value \\
\hline Length of concrete support & $\mathrm{B}_{\mathrm{y}}$ & $4 \mathrm{~m}$ \\
\hline Width of bentonite plug & $\mathrm{S}_{\mathrm{y}}$ & $3 \mathrm{~m}$ \\
\hline Width of access drift & $\mathrm{G}_{\mathrm{y}}$ & $6 \mathrm{~m}$ \\
\hline Length of cell & $\mathrm{A}_{\mathrm{y}}$ & $30 \mathrm{~m}$ \\
\hline Height of access and main drifts & $\mathrm{G}_{\mathrm{z}}$ & $6 \mathrm{~m}$ \\
\hline Height of the calculation domain & $\mathrm{L}_{\mathrm{z}}$ & $130 \mathrm{~m}$ \\
\hline Diameter of cell & $\mathrm{A}_{\mathrm{x}}$ & $0.7 \mathrm{~m}$ \\
\hline Centre distance between cells & $\mathrm{E}_{\mathrm{x}}$ & $12 \mathrm{~m}$ \\
\hline Distance between cell and main drift & $\mathrm{T}_{\mathrm{x}}$ & $20 \mathrm{~m}$ \\
\hline Half-width of the main drift & $\mathrm{G}_{\mathrm{x}}$ & $3 \mathrm{~m}$ \\
\hline Length of the calculation domain & $\mathrm{L}_{\mathrm{x}}$ & $392 \mathrm{~m}$ \\
\hline Width of the calculation domain & $\mathrm{L}_{\mathrm{y}}$ & $100 \mathrm{~m}$ \\
\hline Length of the seal & $\mathrm{S}_{\mathrm{y}}$ & $10 \mathrm{~m}$ \\
\hline & $\mathrm{R}_{\mathrm{y}}$ & $10 \mathrm{~m}$ \\
\hline & $\mathrm{R}_{\mathrm{z}}$ & $62 \mathrm{~m}$ \\
\hline & $\mathrm{R}_{\mathrm{x}}$ & $20 \mathrm{~m}$ \\
\hline & $\mathrm{P}_{\mathrm{x}}$ & $0,65 \mathrm{~m}$ \\
\hline
\end{tabular}

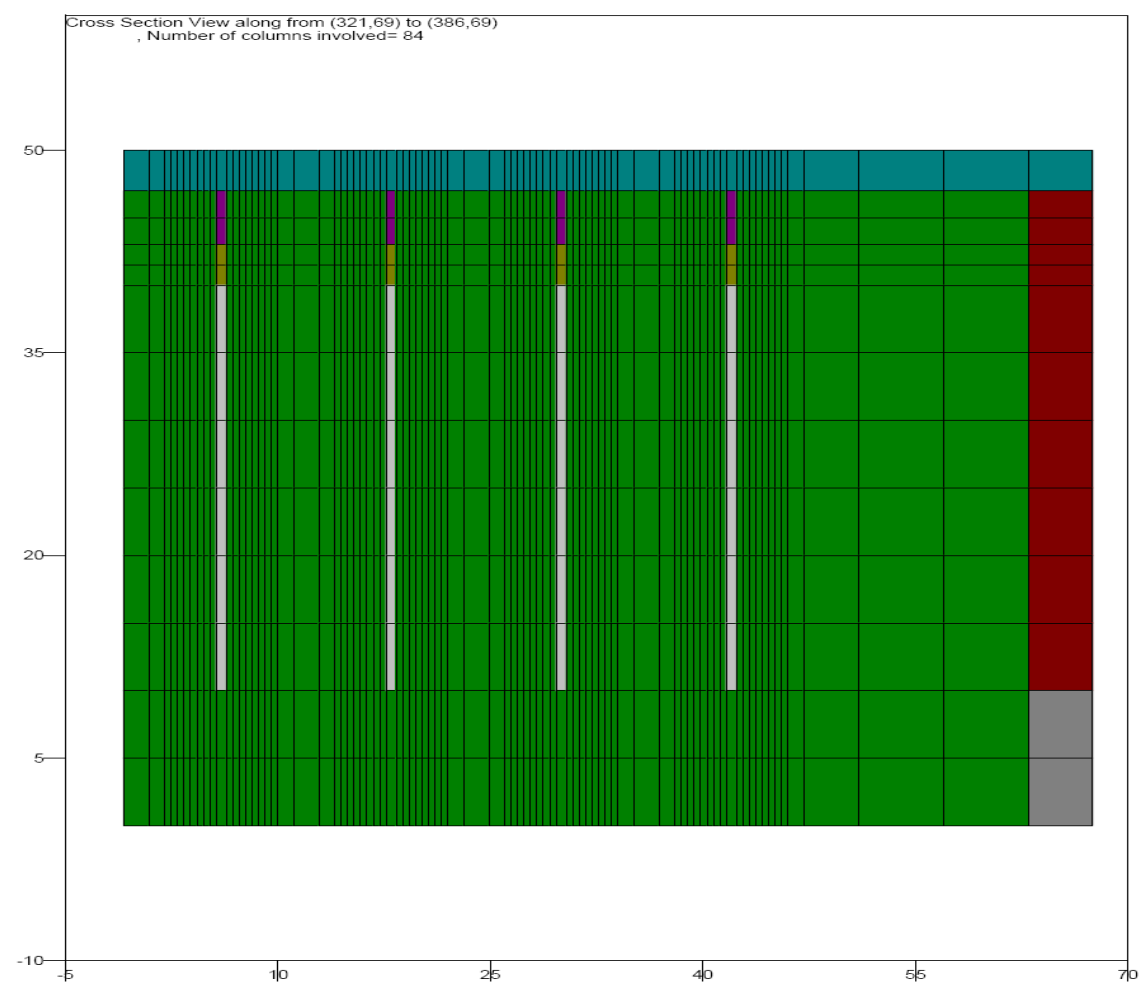

Figure 6-9 Mesh discretization along XY plan (zoom on the main drift and the for adjacent emplacement cells). 


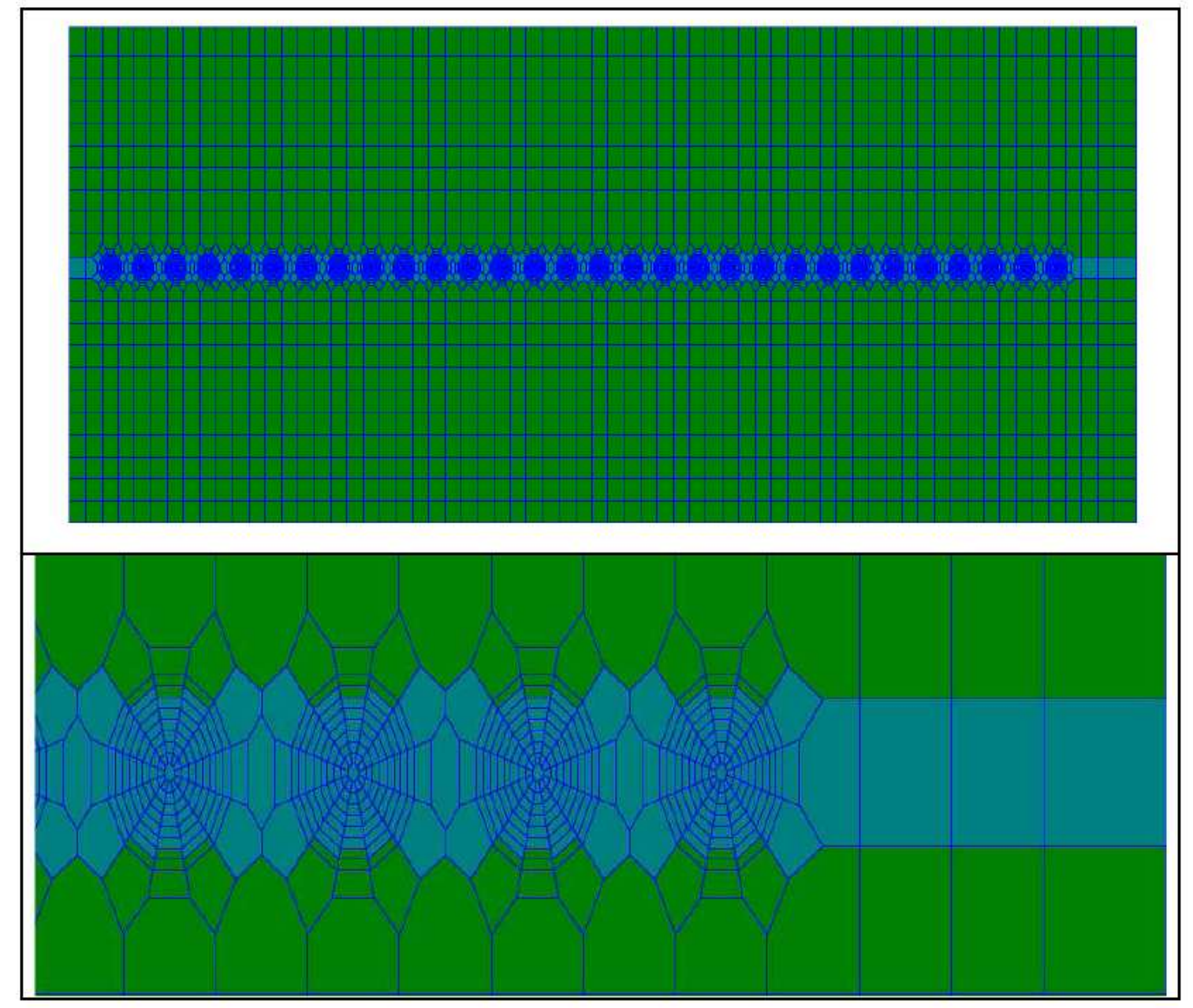

Figure 6-10 Mesh discretization along XZ plane. Upper: the whole plane; lower: refined mesh around waste cells. 


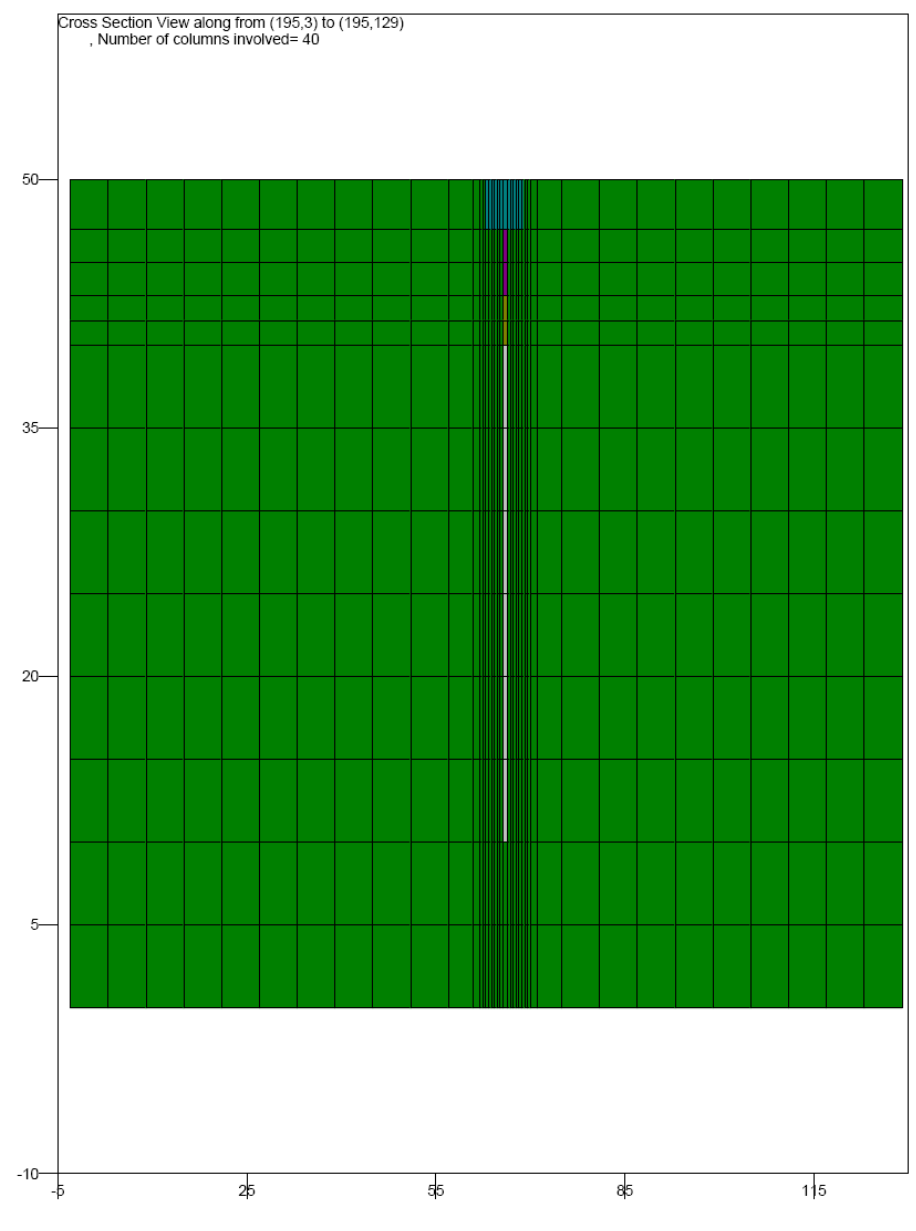

Figure 6-11 Mesh discretization along YZ plane.

Boundary conditions of the model include constant water pressure at top $(4.21 \mathrm{e} 06 \mathrm{~Pa})$, bottom $(5.51 \mathrm{e} 06 \mathrm{~Pa})$, and mouth of the main drift $(4.85 \mathrm{e} 06 \mathrm{~Pa})$. Four sides of the domain are no-flow boundaries. The gas generation rate is $100 \mathrm{~mol} / \mathrm{year} / \mathrm{cell}$ during the first 4,500 years, $15 \mathrm{~mol} /$ year/cell during 4,500-20,000 years, and $1 \mathrm{~mol} / \mathrm{year} / \mathrm{cell}$ during 20,000-50,000 years. No gas is generated after 50,000 years. The model has an initial water saturation equal to 1.0 in the Callovo-Oxfordian formation and equal to 0.7 in the other media. The initial pressure is distributed linearly in accordance with the pressure gradient between the roof and the wall of the Callovo-Oxfordian formation. In partiallysaturated materials, the initial gas pressure is equal to 1 atmosphere. The water pressure is deduced from the gas pressure and the saturation pressure by applying Van Genuchten models associated with each material. For more information on the model specifications the user may refer to the ANDRA report. 
Table 6-3 Physical parameters for the rocks (From ANDRA report)

\begin{tabular}{|c|c|c|c|c|c|c|}
\hline \multirow{2}{*}{$\begin{array}{l}\text { Parameter } \\
\text { (at } 30^{\circ} \mathrm{C} \text { ) }\end{array}$} & \multicolumn{6}{|c|}{ Materials } \\
\hline & Cell & Concrete & Bentonite & Backfill & Seal & $\mathrm{COX}$ \\
\hline $\mathrm{K}_{\mathrm{v}}\left[\mathrm{m}^{2}\right]$ & 0 & $1.010^{-18}$ & $1.010^{-20}$ & $6.010^{-10}$ & $1.010^{-18}$ & $5.010^{-21}$ \\
\hline $\mathrm{K}_{\mathrm{h}}\left[\mathrm{m}^{2}\right]$ & \multicolumn{5}{|c|}{$\mathrm{Kv}=\mathrm{Kh}$} & $5.010^{-20}$ \\
\hline Porosity [\%] & 25 & 30 & 35 & 35 & 30 & 15 \\
\hline $\begin{array}{l}\text { Specific storage } \\
\text { coefficient }\left[\mathrm{m}^{-1}\right]\end{array}$ & $4.010^{-06}$ & $2.310^{-06}$ & $4.410^{-06}$ & $4.010^{-06}$ & $3.010^{-06}$ & $2.310^{-06}$ \\
\hline \multicolumn{7}{|c|}{ Two-phase flow parameters } \\
\hline $\mathrm{S}_{\mathrm{gr}}[\%]$ & 0 & 0 & 0 & 0 & 0 & 0 \\
\hline $\mathrm{S}_{\mathrm{wr}}[\%]$ & 1 & 1 & 1 & 1 & 1 & 40 \\
\hline \multicolumn{7}{|c|}{ Van Genuchten parameters } \\
\hline $\mathrm{n}[-]$ & 1.5 & 1.54 & 1.61 & 1.42 & 1.5 & 1.49 \\
\hline $\mathrm{P}_{\mathrm{r}}[\mathrm{Pa}]$ & $310^{4}$ & $210^{6}$ & $1610^{6}$ & $610^{5}$ & $510^{6}$ & $1510^{6}$ \\
\hline $\begin{array}{c}\tau \\
\text { (Tortuosity) }\end{array}$ & 1 & 2 & 4.5 & 2 & 2 & 2 \\
\hline
\end{tabular}

Because the model involves water and hydrogen as the two components and two phases, we select EOS5 for the simulation. The model was considered as isothermal case with constant temperature $30^{\circ} \mathrm{C}$. To facilitate simulation run, diffusion effect is neglected for the first 5,000-year simulation. The model was first run to steady-state with specified constant pressure at the domain top, bottom, and main drift mouth. The hydrostatic equilibrium is obtained from the steady-state solution. The initial condition for further gas generation simulations is obtained by incorporating the condition of 0.7 water saturation for some materials as requested into the hydrostatic equilibrium condition. For convenience of the users, we provide all input files needed for gas simulation, including the initial condition file INCON, source/sink file GENER, mesh file MESH, and main input file INFILE. Users may directly use these files for the two phase flow simulations.

Figure 6-12 shows gas pressure at the repository level at time 1000 years and 2000 years. Figure 6-13 and 6-14 show the corresponding simulated water pressure and gas saturation. Figure 6-15and Figure 6-16 show gas pressure and gas saturation changes with time at different locations. 

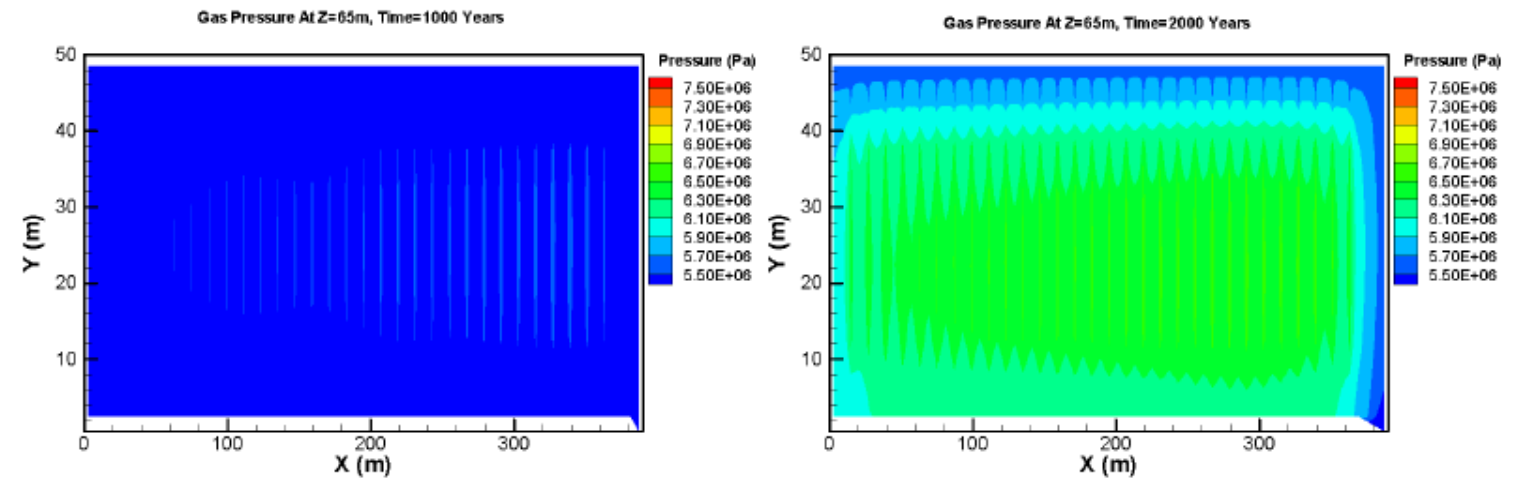

Figure 6-12 Simulated gas pressure at the repository level at times of 1,000 years and 2,000 years.
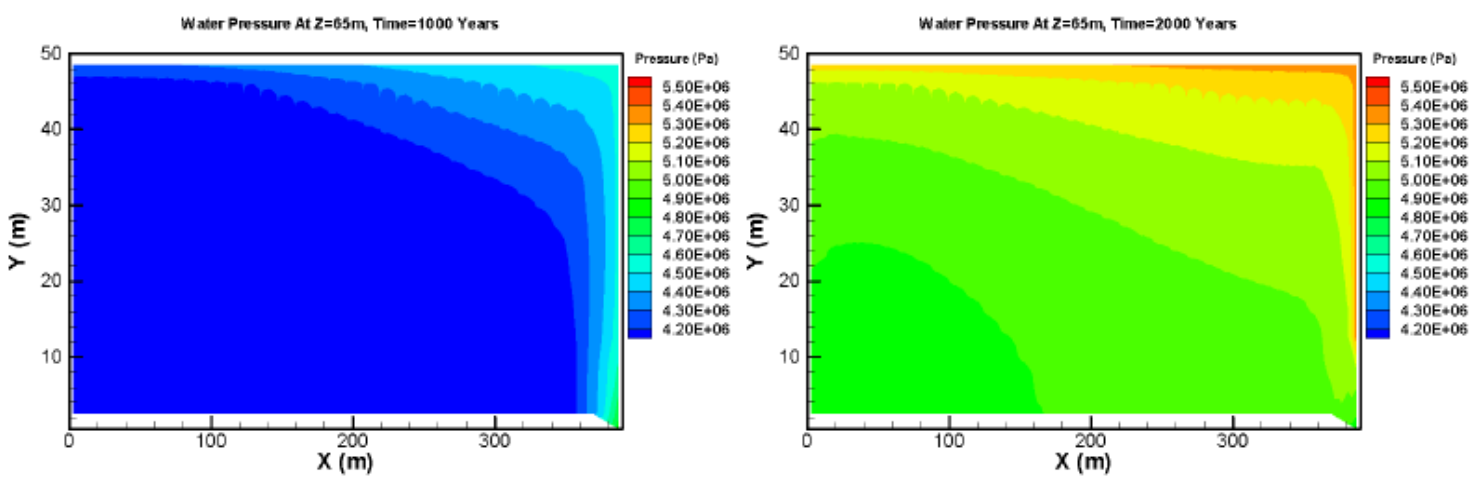

Figure 6-13 Simulated water pressure at the repository level at times of 1,000 years and 2,000 years.
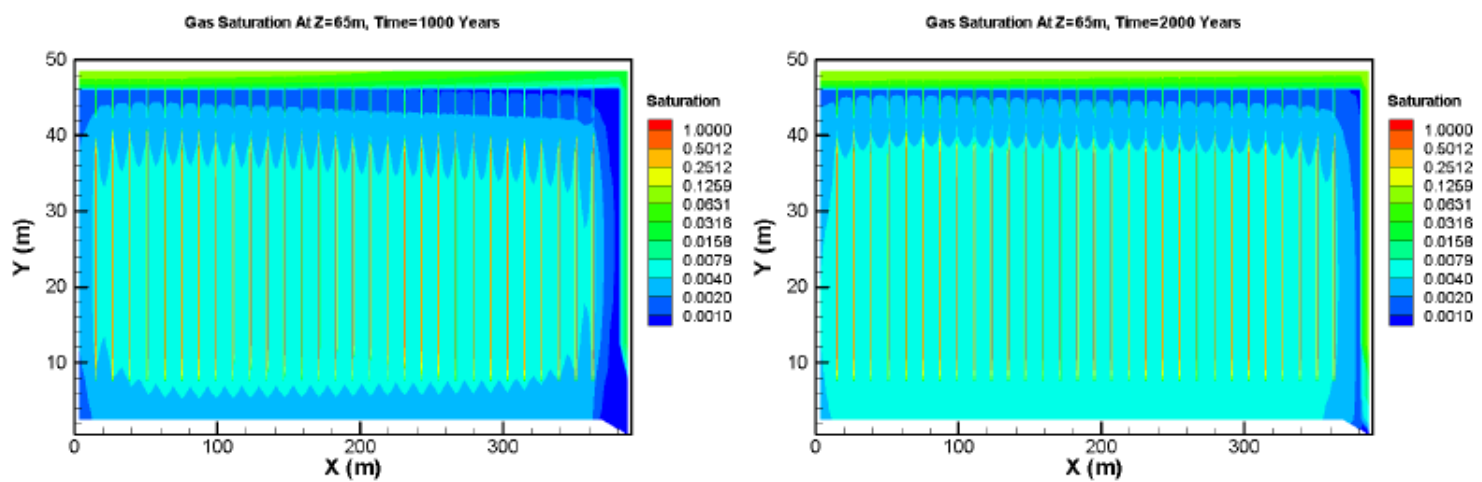

Figure 6-14 Simulated gas saturation at the repository level at times of 1,000 years and 2,000 years. 


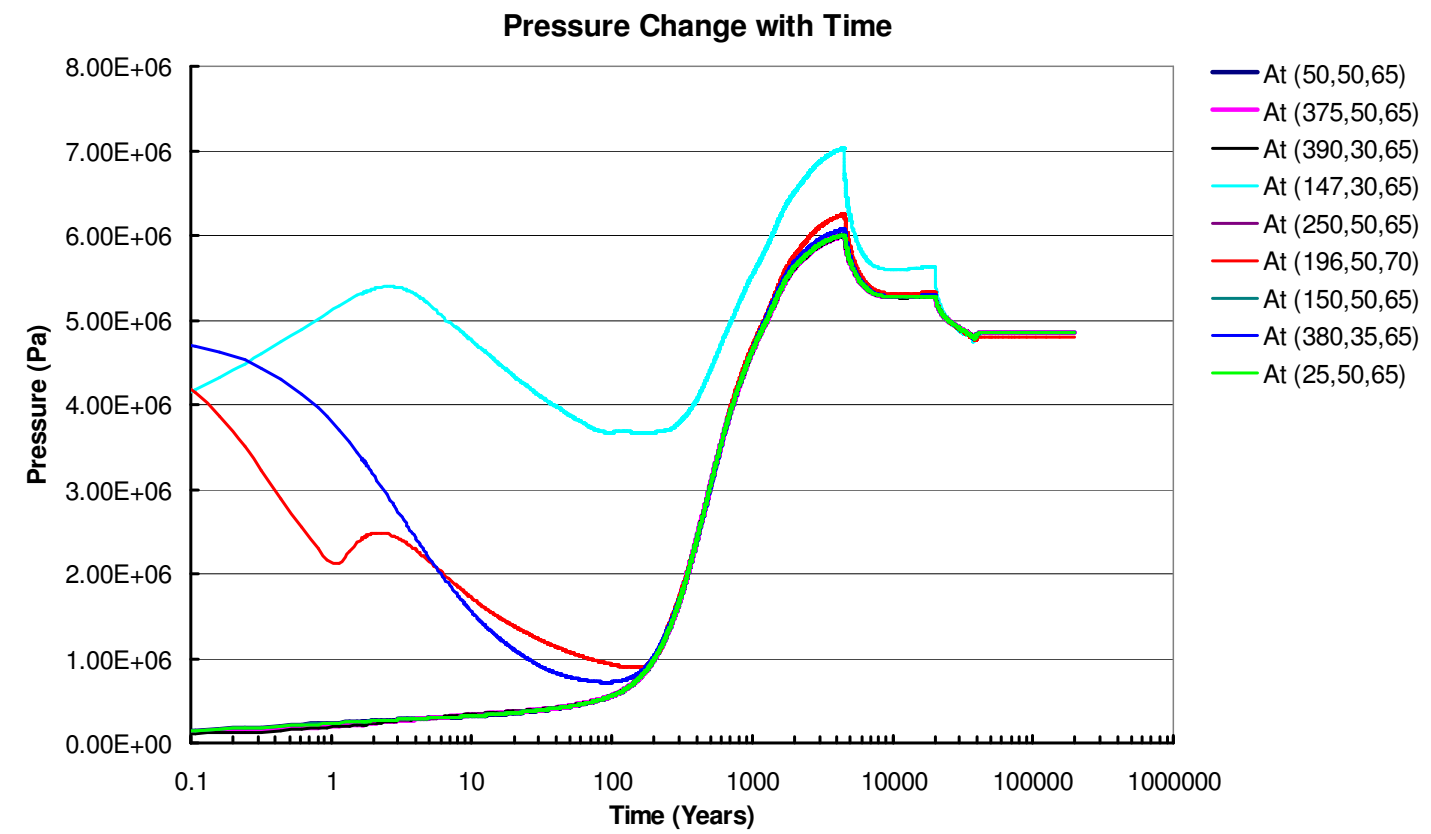

Figure 6-15. Simulated gas (or water under single phase conditions) pressure changes with time at different locations.

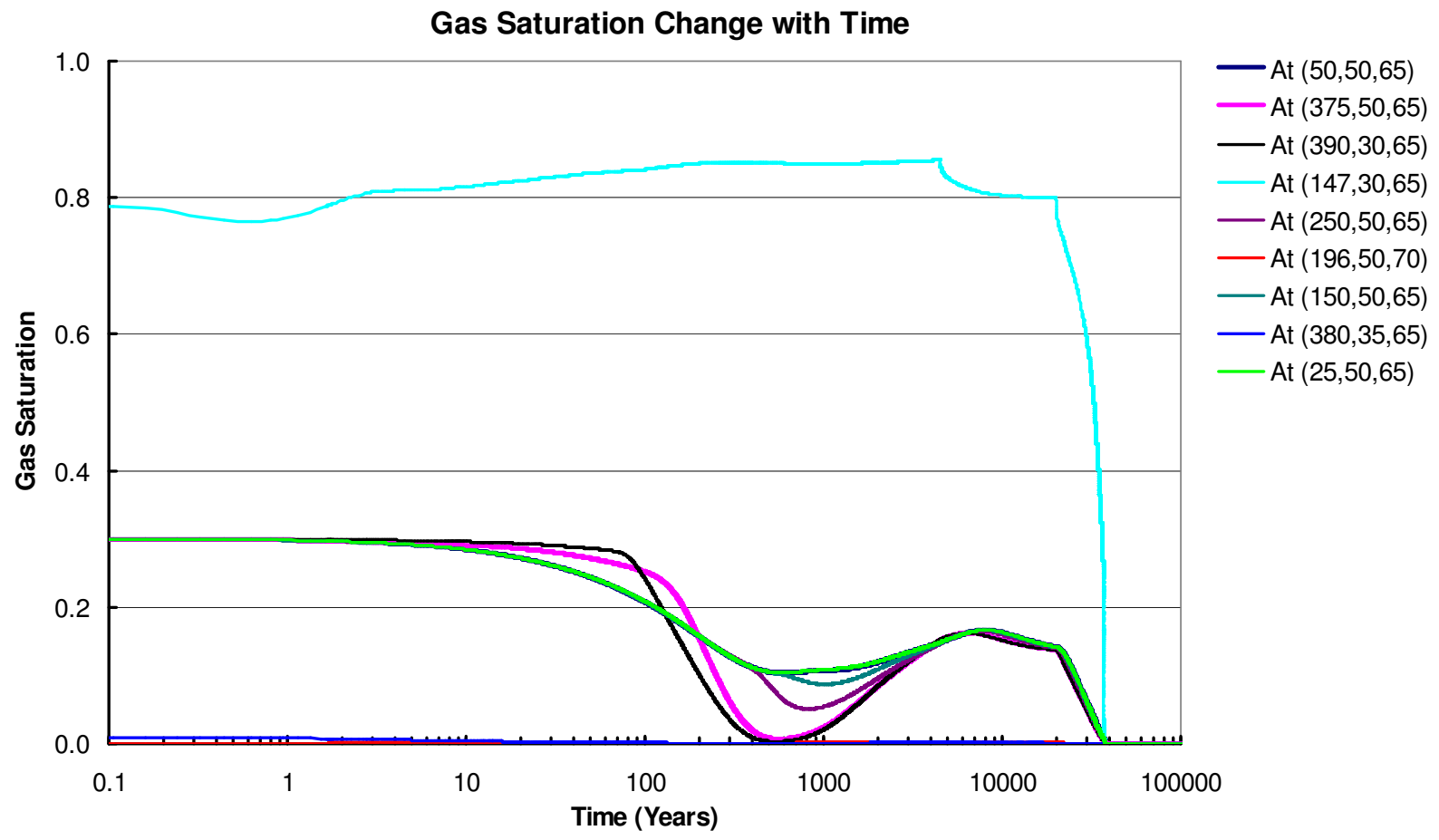

Figure 6-16. Simulated gas saturation changes with time at different locations 
This sample problem provides an example for typical 3D models. The example could be a good template for large-scale field problem simulations. Users may use the provided simulation results in this manual to confirm the correctness of parallel simulation on their computers. This example may also be good for testing the parallel performance of multiCPU computers. Users can look into the details of the model through the input files to learn more details for complex model setup and simulation with the parallel code.

\section{CONCLUDING REMARKS}

A massively parallel simulator, named TOUGH2-MP, for isothermal and nonisothermal flows of multicomponent, multiphase fluids in one, two, and three-dimensional porous and fractured media has been developed. The parallel simulator solves large, sparse linear systems arising from discretization of the partial differential equations for mass and energy balance. A domain decomposition approach is adopted for multiphase flow simulations with coarse-granularity parallel computation. This approach partitions a simulation domain into a number of smaller subdomains. The full model domain, consisting of partitioned subdomains, is still simulated simultaneously by using multiple processes/processors. Each processor is dedicated to the following tasks for the partitioned subdomain: updating thermophysical properties, assembling mass- and energy-balance equations, solving linear equation systems, and performing various other local computations. The linearized equation systems are solved in parallel with a parallel linear solver, using an efficient inter-processor communication scheme.

TOUGH2-MP was developed based on the sequential TOUGH2 V2.0 and V1.4 codes. It was written in Fortran 90 with MPI for parallel implementation. Because the parallel simulator was developed from an existing mature code, it inherits not only simulation functions from the original TOUGH2 code, but also all other features, including input/output format, error handling, and improvements for code stability. These features provide robustness of the parallel code and ease of use to the TOUGH 2 community. TOUGH2-MP is designed to use identical input data, mesh and output files as TOUGH2 V2.0. 
TOUGH2-MP has demonstrated excellent speedup and good scalability. It is more efficient than its sequential counterpart, especially for larger problems. The code provides a powerful tool for tackling larger-scale and more complex problems than can be handled currently by sequential codes. The new simulator enhances modeling capacity in terms of both model size and simulation time by 1-3 orders of the magnitude. It allows for much larger problems to be solved by multiple-process simulation even with a single-processor. The code can make full use of the computing resources of multi-core CPUs. The growing availability of multi-core CPUs will make parallel processing on PCs far more attractive in reservoir simulation practice.

\section{ACKNOWLEDGES}

We thank Nicholaus Halecky for reviewing the manuscript. The prototype of TOUGH2-MP was developed during 1999-2000 through an LDRD project entitled "Development of the high performance TOUGH2 codes". Erik Elmroth and Chris Ding made important contributions to the early phase of the prototype development. The code has been redeveloped, improved, and augmented with more modules while applied to many DOE sponsored projects, especially the Yucca Mountain project sponsored by DOE Civil Radioactive Waste Management program. These supports were provided under Contract No. DE-AC02-05CH11231 with the U.S. Department of Energy. The authors would like to thank their colleagues at LBNL for many discussions and helpful suggestions. Betatesting and evaluation of the code were performed by Robert Podgorney (Idaho National Engineering and Environmental Laboratory), Hajime Yamamoto (Taisei, Japan), Jean Croisé and Mayer Gerhard (AF-Colenco Ltd, Switzerland), Chunmiao Zheng and Song Chen (University of Alabama), Ljubinko Miljkovic (Stanford University), David J. Noy (British Geological Survey), Rainer Senger (Intera, Inc), and others. We thank them for their reports on the code performance and suggestions for improvements. Specially, we are indebted to the late Dr. Stephen White (Industrial Research Limited, New Zealand), who was the first user of this code outside LBNL. He provided a binary searching subroutine which dramatically enhanced the TOUGH2 index searching speed. Thanks also go to ANDRA (French National Radioactive Waste Management Agency) and AF- 
Colenco Ltd, Switzerland for permission to include Couplex-gas Test case 2 in this report as a sample problem. 


\section{REFERENCES}

Andra, 2006: Couplex-gaz benchmark experiment: http://www.andra.fr/IMG/pdf/test_cases2.pdf

Bodvarsson, G.S., Y.S. Wu, K. Zhang, 2003, Development of Discrete Flow Paths in Unsaturated Fractures at Yucca Mountain, Journal Of Contaminant Hydrology, 62$63,23-42$.

Corey, A.T., The Interrelation Between Gas and Oil Relative Permeabilities, Producers Monthly, 38-41, November 1954. NNA.19900720.0036

Croisé, J., and K. Zhang, Exercice Couplex-Gaz: Cas 2a : déchets vitrifies, Report 1101/004, AF-Colenco Ltd, Switzerland, 2008.

Elmroth, E., C. Ding, and Y. S. Wu, High Performance Computations for Large-Scale Simulations of Subsurface Multiphase Fluid and Heat Flow, The Journal of Supercomputing, 18(3), pp. 233-256, 2001.

Fatt, I. and W.A. Klikoff. Effect of Fractional Wettability on Multiphase Flow Through Porous Media, AIME Transactions, 216, 246, 1959. NNA.19900917.0129

Karypsis, G. and V. Kumar, METIS. A Software Package for Partitioning Unstructured Graphs, Partitioning Meshes, and Computing Fill-Reducing Orderings of Sparse Matrices, V4.0. Technical Report, Department of Computer Science, University of Minnesota, 1998.

Leverett, M.C., Capillary Behavior in Porous Solids, Trans. Soc. Pet. Eng. AIME, 142, $152-169,1941$.

Liu, H. H., C. Doughty, and G. S. Bodvarsson, An Active Fracture Model For Unsaturated Flow And Transport In Fractured Rocks, Water Resources Research, 34, 2633-2646, 1998.

Message Passing Forum, A Message-Passing Interface Standard, International Journal of Supercomputing Applications and High performance Computing, 8(3-4), 1994. 
Milly, P.C.D., Moisture and Heat Transport in Hysteretic, Inhomogeneous Porous Media: A Matric-Head Based Formulation and a Numerical Model, Water Resour. Res., Vol. 18, No. 3, pp. 489 - 498, 1982.

Mualem, Y., A New Model for Predicting the Hydraulic Conductivity of Unsaturated Porous Media, Water Resour. Res., Vol. 12(3), pp. 513 - 522, 1976. NNA.19881228.0005

Narasimhan, T. N. and P. A. Witherspoon, An Integrated Finite Difference Method for Analyzing Fluid Flow in Porous Media, Water Resources Research, 12(1), pp. 57-64, 1976.

Narasimhan, T.N., P.A. Witherspoon and A.L. Edwards. Numerical Model for SaturatedUnsaturated Flow in Deformable Porous Media, Part 2: The Algorithm, Water Resour. Res., 14 (2), 255-261, 1978.

Pickens, J.F., R.W. Gillham and D.R. Cameron. Finite Element Analysis of the Transport of Water and Solutes in Tile-Drained Soils, J. of Hydrology, 40, 243-264, 1979.

Pruess, K., GMINC - A Mesh Generator for Flow Simulations in Fractured Reservoirs, Report LBL-15227, Berkeley, California: Lawrence Berkeley National Laboratory, 1983.

Pruess, K., TOUGH User's Guide, Nuclear Regulatory Commission Report NUREG/CR4645; also Lawrence Berkeley Laboratory Report LBL-20700, 1987.

Pruess, K., TOUGH2 - A general-purpose numerical simulator for multiphase fluid and heart flow, Lawrence Berkeley Laboratory Report LBNL-29400, Berkeley, CA, 1991.

Pruess, K. The TOUGH Codes-A Family of Simulation Tools for Multiphase Flow and Transport Processes in Permeable Media, Vadose Zone J., Vol. 3, pp. 738 - 746, 2004.

Pruess, K., and T. N. Narasimhan, A Practical Method for Modeling Fluid and Heat Flow in Fractured Porous Media, Soc. Pet. Eng. J., 25, pp. 14-26, 1985. 
Pruess, K, C. Oldenburg, and G. Moridis, TOUGH2 User's Guide, V2.0. Lawrence Berkeley National Laboratory Report LBNL-43134, Berkeley, CA, 1999.

Pruess, K., and Y. Tsang, 1994, Thermal Modeling for a Potential High-Level Nuclear Waste Repository at Yucca Mountain, Nevada, Lawrence Berkeley Laboratory Report, LBL-35381, UC-600, Lawrence Berkeley National Laboratories, Berkeley, CA

Senger, R., K. Zhang, J. Avis, P. Marschall' Three-dimensional modeling of gas migration in a deep low/intermediate level waste repository (Switzerland), Abs, Computational Methods in Water Resources XVII International Conference, San Francisco, CA, 2008.

Tuminaro, R. S., M. Heroux, S. A. Hutchinson, and J. N. Shadid, Official Aztec user's guide, Ver 2.1, Massively Parallel Computing Research Laboratory, Sandia National Laboratories, Albuquerque, NM, 1999.

Udell, K.S. and J.S. Fitch. Heat and Mass Transfer in Capillary Porous Media Considering Evaporation, Condensation, and Non-Condensible Gas Effects, paper presented at 23rd ASME/AIChE National Heat Transfer Conference, Denver, CO, 1985.

Van Genuchten, M.Th. A Closed-Form Equation for Predicting the Hydraulic Conductivity of Unsaturated Soils, Soil Sci. Soc., Vol. 44, pp. 892 - 898, 1980. NNA.19911009.0008

Verma, A.K., K. Pruess, C.F. Tsang and P.A. Witherspoon. A Study of Two-Phase Concurrent Flow of Steam and Water in an Unconsolidated Porous Medium, Proc. 23rd National Heat Transfer Conference, Am. Society of Mechanical Engineers, Denver, CO, 135-143, 1985. NNA.19890713.0198

Wu, Y. S., USERS MANUAL (UM) for T2R3D, Version 1.4., STN: 10006.14.00, Research Report, Earth Sciences Division, Lawrence Berkeley National Laboratory, Berkeley, CA, 1999. 
Wu, Y. S., USERS MANUAL (UM) for TOUGH2, Version 1.4, STN:10007-1.4-01, Research Report, Earth Sciences Division, Lawrence Berkeley National Laboratory, Berkeley, CA, 2000.

Wu, Y. S., C. F. Ahlers, P. Fraser, A. Simmons, and K. Pruess, Software Qualification of Selected TOUGH2 Modules, Research Report, Earth Sciences Division, Lawrence Berkeley National Laboratory, LBL-39490, UC-800, October, 1996.

Wu, Y. S., C. Haukwa, and S. Mukhopadyay, TOUGH2 V1.4 and T2R3D V1.4: Verification and Validation Report and User's Manual, Rev 00, Lawrence Berkeley National Laboratory, Berkeley, CA, 1999.

Wu, Y. S., K. Zhang, C. Ding, K, Pruess, and G. S. Bodvarsson, An Efficient ParallelComputing Method for Modeling Nonisothermal Multiphase Flow and Multicomponent Transport in Porous and Fractured Media, LBNL-47937, Advances in Water Resources, Vol. 25, pp.243-261, 2002

Yamamoto, H., K. Zhang, K. Karasaki, and A. Marui, 2007, Impact of large-scale geologic $\mathrm{CO} 2$ storage on regional groundwater systems -Numerical simulation using parallelized code, Abs J253-008, Japan Geosciences Union Meeting 2007.

Zhang, K., USERS MANUAL for TOUGH2-MP, Version 1.0., STN: 10007.1.0.00, Research Report, Earth Sciences Division, Lawrence Berkeley National Laboratory, Berkeley, CA, 2003.

Zhang K., C. Doughty, YS Wu, and K. Pruess, 2007, Efficient Parallel Simulation of $\mathrm{CO}_{2}$ Geologic Sequestration in Saline Aquifers, Paper SPE 106026, Proceedings of the 2007 SPE Reservoir Simulation Symposium, Houston, Texas.

Zhang, K., G. J. Moridis, Y. S. Wu, and K. Pruess, A domain decomposition approach for large-scale simulations of flow processes in hydrate-bearing geologic media, Proceedings of the 6th International Conference on Gas Hydrates (ICGH 2008), Vancouver, British Columbia, CANADA, July 6-10, 2008

Zhang K. and YS Wu, 2006, Enhancing Scalability and Efficiency of the TOUGH2_MP 
for Linux Clusters, Proceedings of TOUGH symposium 2006, Berkeley, CA

Zhang, K., Y. S. Wu, and G. S. Bodvarsson, Massively Parallel Computing Simulation of Fluid Flow in the Unsaturated Zone of Yucca Mountain, Nevada, LBNL-48883, Journal of Contaminant Hydrology, pp.381-399, 2003a.

Zhang, K, Y. S. Wu, C. Ding, K. Pruess, and E. Elmroth, Parallel Computing Techniques for Large-Scale Reservoir Simulation of Multi-Component and Multiphase Fluid Flow, Paper SPE 66343, Proceedings of the 2001 SPE Reservoir Simulation Symposium, Houston, Texas, 2001.

Zhang, K., Y.S. Wu, C. Ding, and K. Pruess, 2003, TOUGH2_MP: A parallel Version of TOUGH2, Proceedings of TOUGH symposium 2003, Berkeley, CA.

Zhang, K., Y. S. Wu, and L. Pan, Temporal Damping Effect of the Yucca Mountain Fractured Unsaturated Rock on Transient Infiltration Pulses, LBNL-57539, Journal of Hydrology, Vol. 327, pp.235-248, 2006.

Zhang, K., H. Yamamoto, and K. Pruess, TMVOC-MP: A Parallel Numerical Simulator for Three-Phase Non-isothermal Flows of Multicomponent Hydrocarbon Mixtures in Porous/Fractured Media, Report LBNL-63827, Lawrence Berkeley National Laboratory, Berkeley, CA, 2008 


\section{APPENDIX A. RUNNING TOUGH2-MP ON MULTIPLE- CORE PCs}

\section{A.1 INSTALLING MPICH2}

1. Main MPICH homepage:

http://www-unix.mcs.anl.gov/mpi/

2. Download the Win32IA32 version of MPICH2 from:

http://www-unix.mcs.anl.gov/mpi/mpich2/

3. Run the executable, mpich2-1.0.3-1-win32-ia32.msi (or a more recent version). Most likely it will result in the following error:

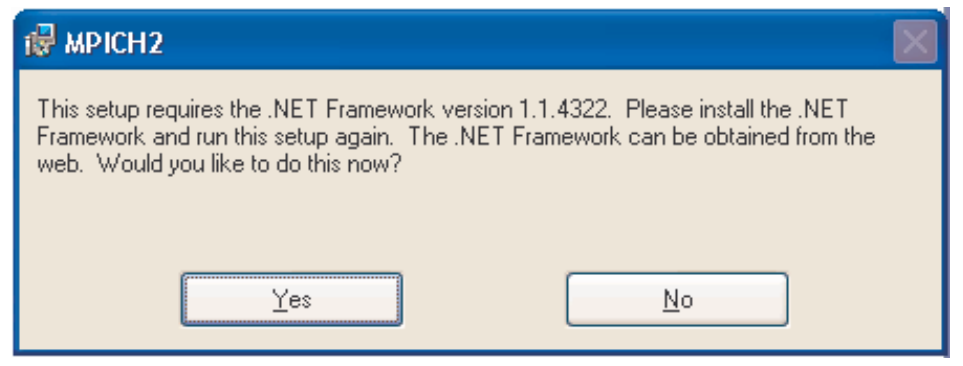

If you follow the link to download the .NET Framework it will download version 2.0, which is not compatible with MPICH2. To download version1.use this link:

http://www.microsoft.com/downloads/details.aspx?FamilyId=262D25E3-F589-4842-

\section{7-034D1E7CF3A3\&displaylang=en}

4. Install the .NET Framework program

5. Install the MPICH2 executable. Write down the passphrase for future reference.

The passphrase must be consistent across a network.

6. Add the MPICH2 path to Windows:

A. Right click "My Computer" and pick properties

B. Select the Advanced Tab

C. Select the Environment Variables button

D. Highlight the path variable under System Variables and click edit. Add "C: $1 \mathrm{MPICH} 2 \mathrm{bin}$ " to the end of the list, make sure to separate this from the prior path with a semicolon.

7. Run the example executable to ensure correct installation.

mpiexec $-\mathrm{n} 2$ cpi.exe 
8. If installed on a dual processor machine, verify that both processors are being utilized by examining "CPU Usage History" in the Windows Task Manager.

9. The first time each session mpiexec is run it will ask for username and password. To prevent being asked for this in the future, this information can be encrypted into the Windows registry by running:

mpiexec-register

The username and password are your Windows logon information.

\section{A.2. Running TOUGH2-MP}

- Make sure that "C:Impich2lbin" is in your window working path.

- Copy input file(s) to the working directory. The format of input files is the same as the input files for standard version TOUGH2. Different from the original TOUGH2, the main input file must be named "INFILE" (case sensitive). The parallel version: (a) does not support simplified mesh format; (b) does not support inactive elements; large volume elements must be used to replace inactive elements; (c) requires to remove previously saved MESHA and MESHB files if MESH was changed.

- An optional input file "PARAL.prm" may be needed for extremely large models (more than 0.5 million grid blocks), provide your own modeling domain partitioning, or you like to try different Aztec solver options. A template of this file is included in the TOUGH2-MP distribution package.

- Change to the directory where you executable and input data are located and type "mpiexec -n X t2eos3_mp". The executable can be in a different directory and run through specifying its path. $\mathrm{X}$ is the number of processes (i.e., the number of subdomains into which the flow systems is partitioned) and t2eos3_mp is the name of the executable. The first time you run in a given logon session you will be asked for your userid and password. These are you Windows XP logon and password. Users are encouraged to experiment with specifying more processes than the number of processors that are available. 


\title{
APPENDIX B. RELATIVE PERMEABILITY FUNCTIONS
}

\author{
$\mathrm{IRP}=1 \quad$ Linear functions \\ $\mathrm{k}_{\mathrm{rl}}$ increases linearly from 0 to 1 in the range \\ $\mathrm{RP}(1) \leq \mathrm{S}_{1} \leq \mathrm{RP}(3) ;$ \\ $\mathrm{k}_{\mathrm{rg}}$ increases linearly from 0 to 1 in the range \\ $\mathrm{RP}(2) \leq \mathrm{S}_{\mathrm{g}} \leq \mathrm{RP}(4)$ \\ Restrictions: $\mathrm{RP}(3)>\mathrm{RP}(1)$; $\mathrm{RP}(4)>\mathrm{RP}(2)$. \\ $\mathrm{IRP}=2 \quad \mathrm{k}_{\mathrm{rl}}=\mathrm{S}_{1} * * \mathrm{RP}(1)$ \\ $\mathrm{k}_{\mathrm{rg}}=1$. \\ IRP $=3 \quad$ Corey's curves (1954)

$$
\begin{gathered}
\mathrm{k}_{\mathrm{rl}}=\hat{\mathrm{S}}^{4} \\
\mathrm{k}_{\mathrm{rg}}=(1-\hat{\mathrm{S}})^{2}\left(1-\hat{\mathrm{S}}^{2}\right)
\end{gathered}
$$ \\ where $\hat{S}=\left(S_{l}-S_{1 r}\right) /\left(1-S_{1 r}-S_{g r}\right)$ \\ with $\mathrm{S}_{\mathrm{lr}}=\mathrm{RP}(1) ; \mathrm{S}_{\mathrm{gr}}=\mathrm{RP}(2)$ \\ Restrictions: $\mathrm{RP}(1)+\mathrm{RP}(2)<1$ \\ IRP $=4 \quad$ Grant's curves

$$
\begin{aligned}
\mathrm{k}_{\mathrm{rl}} & =\hat{\mathrm{S}}^{4} \\
\mathrm{k}_{\mathrm{rg}} & =1-\mathrm{k}_{\mathrm{rl}}
\end{aligned}
$$ \\ where $\hat{S}=\left(S_{1}-S_{l r}\right) /\left(1-S_{l r}-S_{g r}\right)$ \\ with $\mathrm{S}_{\mathrm{lr}}=\mathrm{RP}(1) ; \mathrm{S}_{\mathrm{gr}}=\mathrm{RP}(2)$ \\ Restrictions: $\operatorname{RP}(1)+\mathrm{RP}(2)<1$ \\ $\mathrm{IRP}=5 \quad$ All phases perfectly mobile \\ $\mathrm{k}_{\mathrm{rg}}=\mathrm{k}_{\mathrm{rl}}=1$ for all saturations; no parameters \\ IRP $=6 \quad$ Functions of Fatt and Klikoff (1959)
}




$$
\begin{gathered}
\mathrm{k}_{\mathrm{rl}}=\left(\mathrm{S}^{*}\right)^{3} \\
\mathrm{k}_{\mathrm{rg}}=\left(1-\mathrm{S}^{*}\right)^{3}
\end{gathered}
$$

where $\mathrm{S}^{*}=\left(\mathrm{S}_{1}-\mathrm{S}_{\mathrm{lr}}\right) /\left(1-\mathrm{S}_{\mathrm{lr}}\right)$

with $\mathrm{S}_{\mathrm{Ir}}=\mathrm{RP}(1)$.

Restriction: $\mathrm{RP}(1)<1$.

IRP $=7 \quad$ van Genuchten-Mualem model (Mualem, 1976; van Genuchten, 1980)

$$
k_{\mathrm{rl}}=\left\{\begin{aligned}
\sqrt{\mathrm{S}^{*}}\left\{1-\left(1-\left[\mathrm{S}^{*}\right]^{1 / \lambda}\right)^{\lambda}\right\}^{2} & \text { if } \mathrm{S}_{1}<\mathrm{S}_{\mathrm{ls}} \\
1 & \text { if } \mathrm{S}_{1} \geq \mathrm{S}_{\mathrm{ls}}
\end{aligned}\right.
$$

Gas relative permeability can be chosen as one of the following three forms, the second of which is due to Corey (1954)

$$
\mathrm{k}_{\mathrm{rg}}=\left\{\begin{array}{r}
1-\mathrm{k}_{\mathrm{rl}} \text { if } \mathrm{S}_{\mathrm{gr}}=0, \quad \text { and } \mathrm{RP}(4)=\mathrm{RP}(5)=0 \\
(1-\hat{\mathrm{S}})^{2}\left(1-\hat{\mathrm{S}}^{2}\right) \text { if } \mathrm{S}_{\mathrm{gr}}>0, \mathrm{RP}(4)>0 \text {, and } \mathrm{RP}(5)=0 \\
\left(1-\mathrm{S}^{*}\right)^{2}\left(1-\mathrm{S}^{\frac{*+\gamma}{\gamma}}\right) \text { with } \gamma=\frac{\lambda}{1-\lambda}, \quad \text { if } \mathrm{S}_{\mathrm{gr}}=0 \cdots \text { and } \mathrm{RP}(5)>0
\end{array}\right.
$$

subject to the restriction $0 \leq \mathrm{k}_{\mathrm{rl}}, \mathrm{k}_{\mathrm{rg}} \leq 1$

Here, $S^{*}=\left(S_{1}-S_{l r}\right) /\left(S_{l s}-S_{l r}\right), \quad \hat{S}=\left(S_{1}-S_{l r}\right) /\left(1-S_{l r}-S_{g r}\right)$

Parameters: $\quad \mathrm{RP}(1)=\lambda$

$$
\begin{aligned}
& \mathrm{RP}(2)=\mathrm{S}_{\mathrm{lr}} \\
& \mathrm{RP}(3)=\mathrm{S}_{\mathrm{ls}} \\
& \mathrm{RP}(4)=\mathrm{S}_{\mathrm{gr}} \\
& \mathrm{RP}(5)=\text { switching parameter }
\end{aligned}
$$

Notation: Parameter $\lambda$ is $m$ in van Genuchten's notation, with $m=1-1 / n$; parameter $\mathrm{n}$ is often written as $\beta$.

IRP $=8 \quad$ Function of Verma et al. (1985) 


$$
\begin{gathered}
\mathrm{k}_{\mathrm{rl}}=\hat{\mathrm{S}}^{3} \\
\mathrm{k}_{\mathrm{rg}}=\mathrm{A}+\mathrm{B} \hat{\mathrm{S}}+\mathrm{C} \hat{\mathrm{S}}^{2}
\end{gathered}
$$

where

$$
\hat{S}=\left(S_{1}-S_{l r}\right) /\left(S_{l s}-S_{l r}\right)
$$

Parameters as measured by Verma et al. (1985) for steam-water flow in an unconsolidated sand:

$$
\mathrm{S}_{\mathrm{lr}}=\mathrm{RP}(1)=0.2
$$$$
\mathrm{S}_{\mathrm{ls}}=\mathrm{RP}(2)=0.895
$$

$$
\begin{aligned}
& A=\operatorname{RP}(3)=1.259 \\
& B=\operatorname{RP}(4)=-1.7615 \\
& C=\operatorname{RP}(5)=0.5089
\end{aligned}
$$

\section{$\operatorname{IRP}=9,10 \quad$ ECM function (Pruess and Tsang, 1994)}

These two options are the original effective continuum model (ECM), which use a threshold liquid saturation concept, defined as

$$
\mathrm{S}_{\mathrm{th}}=\frac{\phi_{\mathrm{m}}}{\phi_{\mathrm{m}}+\phi_{\mathrm{f}}}
$$

where both $\phi_{\mathrm{m}}$ and $\phi_{\mathrm{f}}$ are void fractions or porosities for matrix and fractures respectively, defined in terms of the bulk volume of formation.

The only difference between IRP $=9$ and $=10$ is that option of IRP $=9$ handles isotropic permeability cases and IRP $=10$ handles anisotropic permeability scenarios. In general, the two ECM relative permeability functions need (1) matrix continuum and fracture continuum permeability and (2) a special capillary function (defined in ICP $=8$ in Appendix VI). It is assumed that PER(i) and PERF(i), input in ROCKS, are absolute continuum permeability of matrix and fractures $(i=1,2,3)$, respectively, along the three principal axes or directions, as defined in CONNE. See Table B.1 for parameter definition 
Table B.1. Definition of parameters for IRP=9 and 10 with ECM relative permeability functions.

\begin{tabular}{|l|l|l|}
\hline $\mathrm{IRP}=$ & 9 & for ECM option in isotropic fracture systems. \\
\hline $\mathrm{IRP}=$ & 10 & for ECM option in anisotropic fracture systems. \\
\hline $\mathrm{RP}(1)=$ & $\mathrm{M}$ & of van Genuchten's function for matrix. \\
\hline $\mathrm{RP}(2)=$ & $\mathrm{S}_{l r}$ & residual liquid saturation in matrix. \\
\hline $\mathrm{RP}(3)=$ & $\mathrm{M}$ & of van Genuchten's function for fractures. \\
\hline $\mathrm{RP}(4)=$ & $\mathrm{S}_{l r}$ & residual liquid saturation in fractures. \\
\hline $\mathrm{RP}(5)=$ & $\mathrm{k}_{\mathrm{f}} / \mathrm{k}_{\mathrm{m}}$ & $\begin{array}{l}\text { ratio of fracture and matrix permeabilities, used only } \\
\text { for isotropic properties of fracture-matrix systems. }\end{array}$ \\
\hline $\mathrm{RP}(6)=$ & $\mathrm{S}_{t h}$ & Threshold liquid saturation. \\
\hline $\mathrm{RP}(7)=$ & $1-\phi_{f}$ & $\phi_{f}$ is fracture porosity. \\
\hline
\end{tabular}

IRP = $11 \quad$ Generalized ECM function (Wu et al. 1996; Wu 2000)

This is a generalized ECM formulation, which relies only on thermodynamic equilibrium assumption for fracture and matrix systems (Wu, 2000). The generalized ECM relative permeability functions need (1) matrix continuum and fracture continuum permeability and (2) a special capillary function (defined in ICP $=9$ in Appendix VI). It is assumed that PER(i) and PERF(i), input in ROCKS, are absolute continuum permeability of matrix and fractures $(i=1,2,3)$, respectively, along the three principal axes or directions, as defined in CONNE. Table B. 2 defines the parameters for the ECM relative permeability function. 
Table B.2 Definition of parameters for IRP=11 with generalized ECM relative permeability functions.

\begin{tabular}{|l|l|l|}
\hline $\mathrm{IRP}=$ & 11 & For generalized ECM option. \\
\hline $\mathrm{RP}(1)=$ & $\mathrm{m}_{\mathrm{m}}$ & Of van Genuchten's function for matrix. \\
\hline $\mathrm{RP}(2)=$ & $\mathrm{S}_{l r}$ & Residual liquid saturation in matrix. \\
\hline $\mathrm{RP}(3)=$ & $\mathrm{m}_{\mathrm{f}}$ & Of van Genuchten's function for fractures. \\
\hline $\mathrm{RP}(4)=$ & $\mathrm{S}_{l r}$ & Residual liquid saturation in fractures. \\
\hline $\mathrm{RP}(5)=$ & & $\begin{array}{l}>0 \mathrm{k}_{\mathrm{rg}}=1.0 \text { - } \mathrm{k}_{\mathrm{rl}} \\
<0 \text { using Corey's function for } \mathrm{k}_{\mathrm{rg}} .\end{array}$ \\
\hline $\mathrm{RP}(6)=$ & $\mathrm{S}_{\mathrm{gr}}$ & Residual gas saturation in matrix. \\
\hline $\mathrm{RP}(7)=$ & $\phi_{f}$ & Fracture continuum porosity \\
\hline
\end{tabular}




\section{APPENDIX C. CAPILLARY PRESSURE FUNCTIONS}

$$
\begin{aligned}
& \mathrm{ICP}=1 \quad \text { Linear function } \\
& P_{\text {cap }}= \begin{cases}-\mathrm{CP}(1) & \text { for } \mathrm{S}_{1} \leq \mathrm{CP}(2) \\
0 & \text { for } \mathrm{S}_{1} \leq \mathrm{CP}(2) \\
-\mathrm{CP}(1) \frac{\mathrm{CP}(3)-\mathrm{S}_{1}}{\mathrm{CP}(3)-\mathrm{CP}(2)} & \text { for } \mathrm{CP}(2)<\mathrm{S}_{1}<\mathrm{CP}(3)\end{cases}
\end{aligned}
$$

Restriction: $\mathrm{CP}(3)>\mathrm{CP}(2)$.

$\mathrm{ICP}=2 \quad$ Function of Pickens et al. (1979)

$$
P_{\text {cap }}=-P_{0}\left\{\ln \left[\frac{A}{B}\left(1+\sqrt{1-B^{2} / A^{2}}\right)\right]\right\}^{1 / x}
$$

with

$$
\begin{aligned}
& A=\left(1+S_{1} / S_{10}\right)\left(S_{10}-S_{1 r}\right) /\left(S_{10}+S_{1 r}\right) \\
& B=1-S_{1} / S_{10}
\end{aligned}
$$

where

$$
\mathrm{P}_{0}=\mathrm{CP}(1) \quad \mathrm{S}_{1 \mathrm{r}}=\mathrm{CP}(2) \quad \mathrm{S}_{10}=\mathrm{CP}(3) \quad \mathrm{x}=\mathrm{CP}(4)
$$

Restrictions: $0<\mathrm{CP}(2)<1 \leq \mathrm{CP}(3) ; \quad \mathrm{CP}(4) \neq 0$

ICP $=3 \quad$ TRUST capillary pressure (Narasimhan et al., 1978)

$$
P_{\text {cap }}= \begin{cases}\sum_{\mathrm{e}}-\mathrm{P}_{0}\left\lfloor\frac{1-S_{1}}{S_{1}-S_{1 r}}\right]^{1 / \eta} & \text { for } S_{1}<1 \\ 0 & \text { for } S_{1}<1\end{cases}
$$

where

$$
\mathrm{P}_{0}=\mathrm{CP}(1) \quad \mathrm{S}_{\mathrm{lr}}=\mathrm{CP}(2) \quad \eta=\mathrm{CP}(3) \quad \mathrm{P}_{\mathrm{e}}=\mathrm{CP}(4)
$$

Restrictions: $\quad \mathrm{CP}(2) \geq 0 ; \quad \mathrm{CP}(3) \neq 0$

ICP $=4 \quad$ Milly's function (Milly, 1982) 


$$
P_{\text {cap }}=-97.783 \times 10^{\mathrm{A}}
$$

with

$$
A=2.26\left(\frac{0.371}{S_{1}-S_{\mathrm{lr}}}-1\right)^{1 / 4}
$$

where $S_{1 \mathrm{r}}=\mathrm{CP}(1)$

Restriction: $\quad \mathrm{CP}(1) \geq 0$.

ICP = $6 \quad$ Leverett's function (Leverett, 1941; Udell and Fitch, 1985)

$$
\mathrm{P}_{\text {cap }}=-\mathrm{P}_{0} \bullet \sigma(\mathrm{T}) \bullet \mathrm{f}\left(\mathrm{S}_{1}\right)
$$

with

$$
\begin{aligned}
& \sigma(T) \text { - surface tension of water (supplied internally in TOUGH2) } \\
& f\left(S_{1}\right)=1.417\left(1-S^{*}\right)-2.120\left(1-S^{*}\right)^{2}+1.263\left(1-S^{*}\right)^{3}
\end{aligned}
$$

where

$$
\mathrm{S}^{*}=\left(\mathrm{S}_{1}-\mathrm{S}_{\mathrm{lr}}\right) /\left(1-\mathrm{S}_{\mathrm{lr}}\right)
$$

Parameters: $\quad \mathrm{P}_{0}=\mathrm{CP}(1) \quad \mathrm{S}_{\mathrm{lr}}=\mathrm{CP}(2)$

Restriction: $0 \leq \mathrm{CP}(2)<1$

ICP $=7 \quad$ van Genuchten function (van Genuchten, 1980)

$$
\mathrm{P}_{\text {cap }}=-\mathrm{P}_{0}\left(\left[\mathrm{~S}^{*}\right]^{-1 / \lambda}-1\right)^{1-\lambda}
$$

subject to the restriction

$$
-\mathrm{P}_{\max } \leq \mathrm{P}_{\text {cap }} \leq 0
$$

Here,

$$
\mathrm{S}^{*}=\left(\mathrm{S}_{1}-\mathrm{S}_{\mathrm{lr}}\right) /\left(\mathrm{S}_{\mathrm{ls}}-\mathrm{S}_{\mathrm{lr}}\right)
$$

Parameters: $\quad \mathrm{CP}(1)=\lambda=1-1 / \mathrm{n}$

$$
\mathrm{CP}(2)=\mathrm{S}_{\mathrm{lr}} \quad \text { (should be chosen smaller than the }
$$

corresponding parameter in the relative permeability function; see note below.)

$$
\begin{aligned}
& \mathrm{CP}(3)=1 / \mathrm{P}_{0} \\
& \mathrm{CP}(4)=\mathrm{P}_{\max }
\end{aligned}
$$




$$
\mathrm{CP}(5)=\mathrm{S}_{\mathrm{ls}}
$$

Notation: Parameter $\lambda$ is $m$ in van Genuchten's notation, with $m=1-1 / n$; parameter $\mathrm{n}$ is often written as $\beta$.

Note on parameter choices: In van Genuchten's derivation (1980), the parameter $\mathrm{S}_{\mathrm{lr}}$ for irreducible water saturation is the same in the relative permeability and capillary pressure functions. As a consequence, for $S_{1}$ $\rightarrow \mathrm{S}_{\text {lr }}$ we have $\mathrm{k}_{\mathrm{rl}} \rightarrow 0$ and $\mathrm{P}_{\text {cap }} \rightarrow-\infty$, which is unphysical because it implies that the radii of capillary menisci go to zero as liquid phase is becoming immobile (discontinuous). In reality, no special capillary pressure effects are expected when liquid phase becomes discontinuous. Accordingly, we recommend always choosing a smaller $S_{1 r}$ for the capillary pressure compared to the relative permeability function.

ICP $=8 \quad$ ECM function (Pruess and Tsang, 1994)

This ECM capillary function should be used with Option IRP=9 or 10 of ECM relative permeability functions. Table C.1 lists the definition of the related parameters.

Table C.1 Definition of parameters for ICP = 8 with ECM capillary pressure functions.

\begin{tabular}{|l|l|l|}
\hline $\mathrm{ICP}=$ & 8 & For effective continuum approach option. \\
\hline $\mathrm{CP}(1)=$ & $\mathrm{m}$ & Of van Genuchten's function for matrix. \\
\hline $\mathrm{CP}(2)=$ & $\mathrm{S}_{l r}$ & Residual liquid saturation in matrix. \\
\hline $\mathrm{CP}(3)=$ & $\alpha$ & $\begin{array}{l}\text { With a unit }\left(\mathrm{Pa}^{-1}\right), \text { van Genuchten's parameter for } \\
\text { matrix. }\end{array}$ \\
\hline $\mathrm{CP}(4)=$ & $\mathrm{P}_{\text {cmax }}$ & Maximum capillary pressure allowed. \\
\hline $\mathrm{CP}(5)=$ & $\mathrm{S}_{\mathrm{s}}$ & Satiated saturation in matrix. \\
\hline $\mathrm{CP}(6)=$ & $\mathrm{S}_{\text {th }}$ & Threshold liquid saturation. \\
\hline
\end{tabular}


\begin{tabular}{|l|l|l|}
\hline $\mathrm{CP}(7)=$ & $\delta$ & Parameter used to considering air entry effects.
\end{tabular}

$\mathrm{ICP}=9 \quad$ Generalized ECM function (Wu et al. 1996, Wu 2000)

The generalized ECM capillary function should be used only with Option

$\mathrm{IRP}=11$ of generalized ECM relative permeability functions. Table C. 2

lists the definition of the related parameters.

Table C.2 Definition of parameters for ICP = 8 with the generalized ECM capillary pressure functions.

\begin{tabular}{|l|l|l|}
\hline $\mathrm{ICP}=$ & 9 & For ECM option. \\
\hline $\mathrm{CP}(1)=$ & $\mathrm{m}_{\mathrm{m}}$ & Of van Genuchten's m for matrix. \\
\hline $\mathrm{CP}(2)=$ & $\mathrm{S}_{l r}$ & Residual liquid saturation in matrix. \\
\hline $\mathrm{CP}(3)=$ & $\alpha_{\mathrm{m}}$ & With a unit $\mathrm{Pa}^{-1}$, van Genuchten's parameter for matrix. \\
\hline $\mathrm{CP}(4)=$ & $\mathrm{P}_{\mathrm{cmax}}$ & Maximum capillary pressure allowed. \\
\hline $\mathrm{CP}(5)=$ & $\mathrm{S}_{\mathrm{lr}}$ & Residual liquid saturation in fractures. \\
\hline $\mathrm{CP}(6)=$ & $\mathrm{m}_{\mathrm{f}}$ & Of van Genuchten's m for fractures. \\
\hline $\mathrm{CP}(7)=$ & $\alpha_{\mathrm{f}}$ & With a unit $\mathrm{Pa}^{-1}$, van Genuchten's Parameter for fractures \\
\hline
\end{tabular}

